\title{
Compatible-Strain Mixed Finite Element Methods for 2D Compressible Nonlinear Elasticity
}

\author{
Arzhang Angoshtari* \\ Mostafa Faghih Shojaei ${ }^{\dagger}$ \\ Arash Yavari ${ }^{\ddagger}$
}

September 26, 2016

\begin{abstract}
In this paper, using the Hilbert complexes of nonlinear elasticity, the approximation theory for Hilbert complexes, and the finite element exterior calculus, we introduce a new class of mixed finite element methods for 2D nonlinear elasticity called compatible-strain mixed finite element methods (CSFEM). We consider a Hu-Washizu-type mixed formulation and choose the displacement, the displacement gradient, and the first Piola-Kirchhoff stress tensor as independent unknowns. We use the underlying spaces of the Hilbert complexes as the solution and test spaces. We discretize the Hilbert complexes and introduce a new class of mixed finite element methods for nonlinear elasticity by using the underlying finite element spaces of the discrete Hilbert complexes. This automatically enforces the trial spaces of the displacement gradient to satisfy the classical Hadamard jump condition, which is a necessary condition for the compatibility of non-smooth displacement gradients. The underlying finite element spaces of CSFEMs are the tensorial analogues of the standard Nédélec and Raviart-Thomas elements of vector fields. These spaces respect the global topologies of the domains in the sense that they can reproduce certain topological properties of the bodies regardless of the refinement level of meshes. By solving several numerical examples, we demostrate that CSFEMs have a good performance for bending problems, in the near incompressible regime, and for bodies with complex geometries. CSFEMs are capable of accurately approximating stresses and perform well in problems that standard enhanced strain methods suffer from the hourglass instability. Moreover, CSFEMs provide a convenient framework for modeling inhomogeneities.
\end{abstract}

\section{Introduction}

Developing well-performing finite element methods for large deformations of solids is a challenging problem. It is well-known that in many important applications such as bending problems, domains with complex geometries, and in the near-incompressible regime, the standard single-field finite element methods for nonlinear elasticity written in terms of the displacement field have poor convergence behavior. It is also well-established that simple extensions of the well-performing methods for small deformations of solids to large deformations can lead to numerical schemes with poor performances due to the appearance of numerical artifacts and unphysical instabilities (see $[1,2,3]$ and references therein).

Numerous approaches have been proposed in the literature for obtaining better numerical methods for large deformations, some of which include: mesh-free methods [4, 5]; the numerical manifold method

\footnotetext{
${ }^{*}$ Department of Civil and Environmental Engineering, The George Washington University, Washington, D.C. 20052. E-mail: aangoshtari@gwu.edu

${ }^{\dagger}$ School of Civil and Environmental Engineering, Georgia Institute of Technology, Atlanta, GA 30332. E-mail: mfsh@gatech.edu

${ }^{\ddagger}$ School of Civil and Environmental Engineering \& The George W. Woodruff School of Mechanical Engineering, Georgia Institute of Technology, Atlanta, GA 30332. E-mail: arash.yavari@ce.gatech.edu.
} 
$[6,7]$; methods based on the enrichment of trial spaces including the partition of unity method $[8,9]$, the generalized finite element method $[10,11,12]$, and the extended finite element method $[13,14,15]$; methods using reduced integration and stabilization $[16,17,18,19]$; and mixed finite element methods $[20,21,22]$.

Mixed finite element methods are based on saddle-point variational principles. For nonlinear elasticity, there are various choices of saddle-point principles such as the two-field Hellinger-Reissner principle and the three-field $\mathrm{Hu}$-Washizu principle, e.g. see $[21, \S 1.5]$. Mixed methods such as enhanced strain methods have good convergence behavior for bending problems and also in the incompressible and near-incompressible regimes. Other features of mixed methods include good accuracy for coarse meshes, no sensitivity against mesh distortions, and simple implementation of nonlinear constitutive relations. Moreover, since the stress is usually considered as an independent variable in mixed methods, it can be computed with higher accuracy. On the other hand, mixed methods are more complicated than standard methods based on single-field formulations and one has to consider several degrees of freedom for each element. It is also well-known that the trial and test spaces of mixed methods need to satisfy certain compatibility conditions, e.g. the Ladyzhenskaya-Babuška-Brezzi condition [23, 24, 25]. Arbitrary selections of trial spaces can lead to numerical artifacts such as the checkerboard instability or the locking effect, e.g. see $[26, \S 4.2]$. Another unphysical instability observed in mixed methods is the hourglass instability of enhanced strain methods [1].

A novel approach for deriving mixed finite element methods for compressible linear elasticity was introduced by Arnold and Winther [27]. They obtained the first stable mixed finite element methods for the displacement-stress formulation of 2D linear elasticity by using the notion of differential complexes. Differential complexes are sequences of linear operators such that the image of each operator is a subset of the kernel of the next operator. The differential complex of linear elasticity introduced by Kröner [28] contains information about topological properties of bodies. Arnold and Winther [27] obtained compatible finite element spaces for the mixed formulation of linear elasticity by appropriately discretizing the linear elasticity complex such that the discrete complexes preserve all the topological information of the linear elasticity complex. By generalizing this approach, Arnold and his coworkers $[29,30]$ showed that it is also possible to obtain stable mixed methods for some linear operators associated to specific classes of differential complexes.

The main goal of this paper is to introduce a new class of mixed finite element methods for $2 \mathrm{D}$ compressible nonlinear elasticity by employing differential complexes. The differential complexes that are suitable for describing the kinematics and the kinetics of large deformations were recently introduced in $[31,32]$. These complexes are written in terms of the displacement, the displacement gradient, and the first Piola-Kirchhoff stress tensor and are closely related to a well-known complex in differential geometry - the de Rham complex. The nonlinear elasticity complexes contain information about topological properties of domains and can be discretized by using the framework of the finite element exterior calculus discussed in $[29,30]$. The resulting discrete complexes contain the same topological information as the original complexes regardless of the refinement level of meshes. Moreover, the underlying spaces of discrete complexes can be generated by the tensorial analogues of some classical edge and face finite elements for vector fields introduced by Raviart and Thomas [33], Brezzi et al. [34], and Nédélec [35].

We consider a Hu-Washizu-type mixed formulation with the displacement, the displacement gradient, and the first Piola-Kirchhoff stress tensor as independent unknowns. We discretize the nonlinear elasticity complexes by using a triangulation of a given body. A new class of mixed finite element methods are then introduced by using the underlying spaces of discrete complexes. We call the resulting methods compatible-strain mixed finite element methods (CSFEM) as displacement gradients are sought in spaces that satisfy the classical Hadamard jump condition for the compatibility of nonsmooth strains. Note that Arnold et al. [29] considered a two-field mixed formulation for compressible 
linear elasticity as it is straightforward to eliminate the linear strains by using the linear constitutive relations. We study the performance of CSFEMs by solving several numerical examples. In particular, we observe that these methods preform well for bending problems, in the near incompressible regime, and for bodies with complex geometries. CSFEMs are also capable of accurately approximating stresses and perform well in problems that standard enhanced strain methods suffer from the hourglass instability. We also show that CSFEMs provide a novel framework for modeling inhomogeneities.

The main difference between CSFEMs and the enhanced strain methods [20] is that the Hadamard jump condition is explicitly imposed on the displacement gradient in CSFEMs. In enhanced strain methods, the displacement gradient is implicitly assumed to be of $L^{2}$-class and consequently, it will not necessarily satisfy the Hadamard jump condition. In this regard, the enhanced displacement gradient can be considered as a way to implicitly impose the Hadamard jump condition.

This paper is organized as follows. In $\S 2$, we introduce the mixed formulation associated to CSFEMs. We first review the Hilbert complexes that describe the kinematics and the kinetics of $2 \mathrm{D}$ nonlinear elasticity in $\S 2.1$. In $\S 2.2$, by using the strong form of the boundary-value problem of nonlinear elastostatics in terms of the displacement, the displacement gradient, and the first Piola-Kirchhoff stress tensor and Green's formula, we write a mixed formulation for 2D nonlinear elastostatics. The solution and the test spaces of this mixed formulation are the underlying spaces of the Hilbert complexes of 2D nonlinear elasticity. In $\S 3$, we discuss the finite element approximations of the above mixed formulation. In $\S 3.1$, we use the finite element exterior calculus to discretize the Hilbert complex of nonlinear elasticity. Consequently, the underlying spaces of the discrete complexes are finite element spaces. We provide a systematic approach for writing these finite element spaces of an arbitrary order. In $\S 3.2$, we use these finite element spaces to introduce CSFEMs. The implementation of CSFEMs is the subject of $\S 3.3$. To study the performance of CSFEMs, we consider several numerical examples in $\S 4$. Finally, some concluding remarks are given in $\S 5$.

\section{The Mixed Formulation for 2D Nonlinear Elasticity}

In this section, we introduce a mixed formulation for 2D nonlinear elasticity, which will be used for obtaining mixed finite element methods. Assume that the reference configuration $\mathcal{B} \subset \mathbb{R}^{2}$ is a bounded domain with the boundary $\partial \mathcal{B}$ and let $\boldsymbol{N}$ be the unit outward normal vector field of $\partial \mathcal{B}$. We assume that $\partial \mathcal{B}$ is (the closure of) the union of disjoint subsets $\Gamma_{d}$ and $\Gamma_{t}$. Let $\left\{\mathrm{X}^{I}\right\}, I=1,2$, be the Cartesian coordinates of $\mathbb{R}^{2}$. For any vector field $\boldsymbol{U}$ and any $\left(\begin{array}{l}2 \\ 0\end{array}\right)$-tensor field $\boldsymbol{T}, \operatorname{grad} \boldsymbol{U}$ and $\operatorname{div} \boldsymbol{T}$ are, respectively, a $\left(\begin{array}{l}2 \\ 0\end{array}\right)$-tensor field and a vector field defined as

$$
(\operatorname{grad} U)^{I J}=U_{, J}^{I}, \text { and }(\operatorname{div} \boldsymbol{T})^{I}=T^{I J}{ }_{, J},
$$

where ", $J$ " denotes $\partial / \partial \mathrm{X}^{J}$ and we use the summation convention on repeated indices. We also have the operators $\mathbf{c}$ and $\mathbf{s}$, with $\mathbf{c}(\boldsymbol{T})$ being a vector field (the 2 D curl operator) and $\mathbf{s}(\boldsymbol{U})$ being a $\left(\begin{array}{l}2 \\ 0\end{array}\right)$-tensor field given by

$$
(\mathbf{c}(\boldsymbol{T}))^{I}=T_{, 1}^{I 2}-T_{, 2}^{I 1}, \text { and }(\mathbf{s}(\boldsymbol{U}))^{I J}=\delta^{1 J} U_{, 2}^{I}-\delta^{2 J} U^{I}{ }_{, 1},
$$

where $\delta^{I J}$ is the Kronecker delta. The above operators describe the kinematics and the kinetics of a motion $\varphi: \mathcal{B} \rightarrow \mathbb{R}^{2}$. More specifically, let $\boldsymbol{U}(\mathbf{X}):=\varphi(\mathbf{X})-\mathbf{X}, \mathbf{X} \in \mathcal{B}$, be the displacement field associated with $\varphi$. Then, $\boldsymbol{K}:=\operatorname{grad} \boldsymbol{U}$ is the displacement gradient and $\mathbf{c}(\boldsymbol{K})=\mathbf{0}$ is the necessary condition for the compatibility of $\boldsymbol{K}$. On the other hand, $\operatorname{div} \boldsymbol{P}=-\boldsymbol{B}$ expresses the static equilibrium equation in terms of the first Piola-Kirchhoff stress tensor $\boldsymbol{P}$ in the presence of the body force $\boldsymbol{B}$. If $\boldsymbol{B}=\mathbf{0}$, then the equilibrium equation $\operatorname{div} \boldsymbol{P}=\mathbf{0}$ is also the necessary condition for the existence of a stress function $\Psi$ such that $\boldsymbol{P}=\mathbf{s}(\mathbf{\Psi})[31]$. As we discuss in the remainder of this section, the 
domains of some weak extensions of the above operators provide suitable spaces for writing a mixed formulation for nonlinear elasticity.

\subsection{Hilbert Complexes for 2D Nonlinear Elasticity}

For any unit vector $\mathbf{Y}, \boldsymbol{T}(\mathbf{Y}):=T^{I J} Y^{J} \mathbf{E}_{I}$ is called the traction vector of $\boldsymbol{T}$ on a surface with unit normal $\mathbf{Y}$, where $\left\{\mathbf{E}_{1}, \mathbf{E}_{2}\right\}$ is the standard orthonormal basis of $\mathbb{R}^{2}$. We write $\boldsymbol{T} \perp \Gamma_{d}$ if $\boldsymbol{T}(\mathbf{Y})=\mathbf{0}$, for any vector $\mathbf{Y} \| \Gamma_{d}$. We write $\boldsymbol{T} \| \Gamma_{t}$ if $\boldsymbol{T}$ has zero traction vector on $\Gamma_{t}$, that is, $\boldsymbol{T}(\mathbf{Y})=0$, where $\mathbf{Y}$ is the unit normal vector field of $\Gamma_{t}$. Let $H^{1}\left(T \mathcal{B}, \Gamma_{d}\right)$ be the space of $H^{1}$ vector fields that vanish on $\Gamma_{d}$. Also consider the following spaces:

$$
\begin{aligned}
& H^{\mathbf{c}}\left(\mathcal{B}, \Gamma_{d}\right):=\left\{\boldsymbol{T} \in L^{2}: \mathbf{c}(\boldsymbol{T}) \in L^{2} \text { and } \boldsymbol{T} \perp \Gamma_{d}\right\}, \\
& H^{\mathbf{d}}\left(\mathcal{B}, \Gamma_{t}\right):=\left\{\boldsymbol{T} \in L^{2}: \operatorname{div} \boldsymbol{T} \in L^{2} \text { and } \boldsymbol{T} \| \Gamma_{t}\right\}
\end{aligned}
$$

We have $H^{\mathbf{c}}\left(\mathcal{B}, \Gamma_{d}\right) \subset H^{\mathbf{c}}(\mathcal{B}):=H^{\mathbf{c}}(\mathcal{B}, \varnothing)$, and $H^{\mathbf{d}}\left(\mathcal{B}, \Gamma_{d}\right) \subset H^{\mathbf{d}}(\mathcal{B}):=H^{\mathbf{d}}(\mathcal{B}, \varnothing)$. The standard $H^{1}$ Sobolev space is a subset of both $H^{\mathbf{c}}$ and $H^{\mathrm{d}}$. The operators grad and $\mathbf{c}$ can be extended to the mappings

$$
\operatorname{grad}: H^{1}\left(T \mathcal{B}, \Gamma_{d}\right) \rightarrow H^{\mathbf{c}}\left(\mathcal{B}, \Gamma_{d}\right) \text {, and } \mathbf{c}: H^{\mathbf{c}}\left(\mathcal{B}, \Gamma_{d}\right) \rightarrow L^{2}(T \mathcal{B}),
$$

where $L^{2}(T \mathcal{B})$ is the space of $L^{2}$ vector fields. It is straightforward to show that $\mathbf{c}(\operatorname{grad} \boldsymbol{Y})=\mathbf{0}$. These facts are more concisely expressed by writing the following Hilbert complex:

$$
0 \longrightarrow H^{1}\left(T \mathcal{B}, \Gamma_{d}\right) \stackrel{\text { grad }}{\longrightarrow} H^{\mathbf{c}}\left(\mathcal{B}, \Gamma_{d}\right) \stackrel{\mathbf{c}}{\longrightarrow} L^{2}(T \mathcal{B}) \longrightarrow 0
$$

where the first arrow on the left indicates a trivial operator that sends zero to zero and the last arrow on the right indicates the zero operator sending the $L^{2}$-space to zero. Similarly, we have $\operatorname{div}(\mathbf{s}(\boldsymbol{Y}))=\mathbf{0}$, and one can also write the following Hilbert complex

$$
0 \longrightarrow H^{1}\left(T \mathcal{B}, \Gamma_{t}\right) \stackrel{\text { s }}{\longrightarrow} H^{\mathbf{d}}\left(\mathcal{B}, \Gamma_{t}\right) \stackrel{- \text { div }}{\longrightarrow} L^{2}(T \mathcal{B}) \longrightarrow 0
$$

The reason for considering - div instead of $\mathbf{d i v}$ in the second Hilbert complex is that (2.2) is written to be the dual complex of $(2.1)$ in the sense discussed in $[32, \S 3.1]$. The importance of these Hilbert complexes is that they contain information about the topological properties of $\mathcal{B}, \Gamma_{d}$, and $\Gamma_{t}[31,32] .{ }^{1}$ Consequently, these complexes provide a deep connection between topological properties of domains and solutions of certain PDEs. Note that the Hilbert complex (2.1) is related to the kinematics of motion, while the Hilbert complex (2.2) is related to the kinetics of motion.

\subsection{The Mixed Formulation of the Governing Equations}

Let $\rho_{0}, \boldsymbol{U}, \boldsymbol{K}$, and $\boldsymbol{P}$ be the mass density of $\mathcal{B}$, the displacement, the displacement gradient, and the first Piola-Kirchhoff stress tensor, respectively. Also suppose that the constitutive relation can be expressed as $\boldsymbol{P}=\widetilde{\boldsymbol{P}}(\boldsymbol{K})$. The boundary-value problem of nonlinear elastostatics can be stated as follows:

\footnotetext{
${ }^{1}$ More precisely, homological properties of these complexes depend on some topological properties of $\mathcal{B}, \Gamma_{d}$, and $\Gamma_{t}$.
} 
Given a body force $\boldsymbol{B}$, a boundary displacement $\overline{\boldsymbol{U}}$ on $\Gamma_{d}$, and a traction vector field $\overline{\boldsymbol{T}}$ on $\Gamma_{t}$, find $(\boldsymbol{U}, \boldsymbol{K}, \boldsymbol{P})$ such that

$$
\begin{aligned}
& \left.\begin{array}{l}
\operatorname{div} \boldsymbol{P}=-\rho_{0} \boldsymbol{B}, \\
\boldsymbol{P}-\widetilde{\boldsymbol{P}}(\boldsymbol{K})=\mathbf{0}, \\
\boldsymbol{K}-\operatorname{grad} \boldsymbol{U}=\mathbf{0},
\end{array}\right\} \quad \text { on } \mathcal{B}, \\
& \boldsymbol{U}=\overline{\boldsymbol{U}}, \quad \text { on } \Gamma_{d}, \\
& \boldsymbol{P}(\boldsymbol{N})=\overline{\boldsymbol{T}}, \quad \text { on } \Gamma_{t} \text {. }
\end{aligned}
$$

Let $\langle\langle\rangle$,$\rangle be the standard inner product of \mathbb{R}^{2}$ and let $\langle\langle,\rangle\rangle_{L^{2}}$ denote the $L^{2}$-inner products of vector and tensor fields, that is, $\left\langle\langle\boldsymbol{Y}, \boldsymbol{Z}\rangle_{L^{2}}:=\int_{\mathcal{B}} Y^{I} Z^{I} d A\right.$ for vector fields and $\langle\boldsymbol{S}, \boldsymbol{T}\rangle_{L^{2}}:=\int_{\mathcal{B}} S^{I J} T^{I J} d A$ for $\left(\begin{array}{l}2 \\ 0\end{array}\right)$-tensor fields. Suppose $\boldsymbol{P} \in H^{\mathbf{d}}(\mathcal{B})$ and $\boldsymbol{B}$ and $\overline{\boldsymbol{T}}$ are of $L^{2}$-class. Green's formula allows one to write

$$
\left\langle\langle\operatorname{div} \boldsymbol{P}, \mathbf{\Upsilon}\rangle_{L^{2}}=-\left\langle\langle\boldsymbol{P}, \operatorname{grad} \mathbf{\Upsilon}\rangle_{L^{2}}+\int_{\partial \mathcal{B}}\langle\langle\boldsymbol{P}(\boldsymbol{N}), \mathbf{\Upsilon}\rangle\rangle d s,\right.\right.
$$

where $\Upsilon$ is an arbitrary $H^{1}$ vector field. Note that $H^{1} \subset H^{\mathbf{d}} \subset L^{2}$, and therefore, the restriction $\left.\boldsymbol{P}\right|_{\Gamma_{t}}$ is not well-defined for an arbitrary $\boldsymbol{P} \in H^{\mathrm{d}}(\mathcal{B})$, in general. Nonetheless, the following statement of the traction boundary condition is still meaningful in $H^{\mathrm{d}}$ :

$$
\int_{\partial \mathcal{B}}\left\langle\langle\boldsymbol{P}(\boldsymbol{N}), \mathbf{\Upsilon}\rangle d s=\int_{\Gamma_{t}}\langle\overline{\boldsymbol{T}}, \mathbf{\Upsilon}\rangle d s, \forall \mathbf{\Upsilon} \in H^{1}\left(T \mathcal{B}, \Gamma_{d}\right)\right.
$$

where $\left.\int_{\partial \mathcal{B}}\langle\boldsymbol{P}(\boldsymbol{N}), \mathbf{\Upsilon}\rangle\right\rangle d s$ is interpreted as the duality pairing between the spaces $H^{-1 / 2}$ and $H^{1 / 2}$, e.g. see [36, page 298] or [37, Theorem 3.4]. Since $H^{1}\left(T \mathcal{B}, \Gamma_{d}\right)$ is dense in the space of $L^{2}$ vector fields, we conclude that (2.3a) holds if and only if



By using (2.4) and the traction boundary condition (2.5), the above condition can be written as

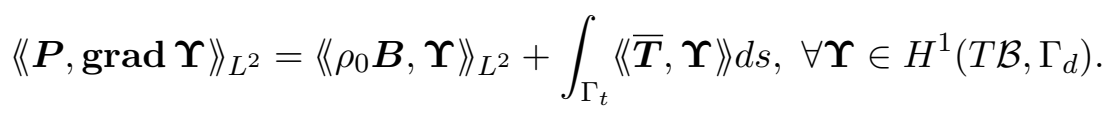

Conversely, Green's formula and the $L^{2}$-density of $H^{1}(T \mathcal{B}, \partial \mathcal{B})$ imply that the above relation gives $(2.3 \mathrm{a})$ and $(2.5)$. On the other hand, since $H^{\mathbf{c}}(\mathcal{B})$ is also dense in the $L^{2}$-space, $(2.3 \mathrm{~b})$ is equivalent to

$$
\left\langle\langle\widetilde{P}(\boldsymbol{K})-\boldsymbol{P}, \boldsymbol{\kappa}\rangle_{L^{2}}=0, \forall \boldsymbol{\kappa} \in H^{\mathbf{c}}(\mathcal{B}) .\right.
$$

Finally, suppose that $\boldsymbol{U} \in H^{1}(T \mathcal{B}), \boldsymbol{K} \in H^{\mathbf{c}}(\mathcal{B})$, and $\overline{\boldsymbol{U}}$ is of $H^{1 / 2}$-class. Note that the condition $\mathbf{c}(\boldsymbol{K})=\mathbf{0}$, is the necessary condition for the compatibility of a displacement gradient $\boldsymbol{K}$. Since $H^{\mathbf{c}}(\mathcal{B})$ is the domain of the operator $\mathbf{c}$ in the $L^{2}$-setting, the assumption $\boldsymbol{K} \in H^{\mathbf{c}}(\mathcal{B})$ is a direct consequence of the compatibility equation for $\boldsymbol{K}$. By using the fact that $H^{\mathbf{d}}\left(\mathcal{B}, \Gamma_{t}\right)$ is $L^{2}$-dense, one concludes that $(2.3 \mathrm{c})$ is equivalent to

$$
\left\langle\langle\boldsymbol{K}-\operatorname{grad} \boldsymbol{U}, \boldsymbol{\pi}\rangle_{L^{2}}=0, \forall \boldsymbol{\pi} \in H^{\mathrm{d}}\left(\mathcal{B}, \Gamma_{t}\right) .\right.
$$

Green's formula suggests that (2.6) and the displacement boundary condition on $\Gamma_{d}$ are equivalent to

$$
\langle\langle\boldsymbol{U}, \operatorname{div} \boldsymbol{\pi}\rangle\rangle_{L^{2}}+\langle\langle\boldsymbol{K}, \boldsymbol{\pi}\rangle\rangle_{L^{2}}=\int_{\Gamma_{d}}\langle\langle\overline{\boldsymbol{U}}, \boldsymbol{\pi}(\boldsymbol{N})\rangle\rangle d s, \forall \boldsymbol{\pi} \in H^{\mathbf{d}}\left(\mathcal{B}, \Gamma_{t}\right) .
$$


Consequently, we consider the following weak formulation of the boundary-value problem (2.3):

Given a body force $\boldsymbol{B}$ of $L^{2}$-class, a boundary displacement $\overline{\boldsymbol{U}}$ on $\Gamma_{d}$ of $H^{1 / 2}$-class, and a boundary traction $\overline{\boldsymbol{T}}$ on $\Gamma_{t}$ of $L^{2}$-class, find $(\boldsymbol{U}, \boldsymbol{K}, \boldsymbol{P}) \in H^{1}(T \mathcal{B}) \times H^{\mathbf{c}}(\mathcal{B}) \times H^{\mathbf{d}}(\mathcal{B})$ such that

$$
\begin{aligned}
\langle\boldsymbol{P}, \operatorname{grad} \boldsymbol{\Upsilon}\rangle_{L^{2}} & =\left\langle\left\langle\rho_{0} \boldsymbol{B}, \boldsymbol{\Upsilon}\right\rangle\right\rangle_{L^{2}}+\int_{\Gamma_{t}}\langle\langle\overline{\boldsymbol{T}}, \boldsymbol{\Upsilon}\rangle\rangle d s, & & \forall \boldsymbol{\Upsilon} \in H^{1}\left(T \mathcal{B}, \Gamma_{d}\right), \\
\langle\widetilde{\boldsymbol{P}}(\boldsymbol{K}), \boldsymbol{\kappa}\rangle\rangle_{L^{2}}-\langle\langle\boldsymbol{P}, \boldsymbol{\kappa}\rangle\rangle_{L^{2}} & =\mathbf{0}, & & \forall \boldsymbol{\kappa} \in H^{\mathbf{c}}(\mathcal{B}), \\
\langle\langle\boldsymbol{U}, \operatorname{div} \boldsymbol{\pi}\rangle\rangle_{L^{2}}+\langle\langle\boldsymbol{K}, \boldsymbol{\pi}\rangle\rangle_{L^{2}} & =\int_{\Gamma_{d}}\langle\langle\overline{\boldsymbol{U}}, \boldsymbol{\pi}(\boldsymbol{N})\rangle\rangle d s, & & \forall \boldsymbol{\pi} \in H^{\mathbf{d}}\left(\mathcal{B}, \Gamma_{t}\right) .
\end{aligned}
$$

Note that the displacement and the traction boundary conditions in the above problem are imposed as natural boundary conditions, that is, they are imposed through the weak formulation and not directly by the solution spaces. The zero displacement boundary condition $\overline{\boldsymbol{U}}=\mathbf{0}$, can be imposed as an essential boundary condition by seeking a solution $(\boldsymbol{U}, \boldsymbol{K}, \boldsymbol{P}) \in H^{1}\left(T \mathcal{B}, \Gamma_{d}\right) \times H^{\mathbf{c}}\left(\mathcal{B}, \Gamma_{d}\right) \times H^{\mathbf{d}}(\mathcal{B})$ with $(\boldsymbol{\Upsilon}, \boldsymbol{\kappa}, \boldsymbol{\pi}) \in H^{1}\left(T \mathcal{B}, \Gamma_{d}\right) \times H^{\mathbf{c}}\left(\mathcal{B}, \Gamma_{d}\right) \times H^{\mathbf{d}}\left(\mathcal{B}, \Gamma_{t}\right)$. In this case, the fact that $\boldsymbol{K} \in H^{\mathbf{c}}\left(\mathcal{B}, \Gamma_{d}\right)$ is a direct consequence of the Hilbert complex (2.1) and the $L^{2}$-density of $H^{\mathbf{c}}\left(\mathcal{B}, \Gamma_{d}\right)$ suggests that one can assume that $\boldsymbol{\kappa} \in H^{\mathbf{c}}\left(\mathcal{B}, \Gamma_{d}\right)$. The zero traction boundary condition $\overline{\boldsymbol{T}}=\mathbf{0}$ can also be treated as an essential boundary condition by assuming $\boldsymbol{P} \in H^{\mathbf{d}}\left(\mathcal{B}, \Gamma_{t}\right)$. In particular, note that for problems with zero displacement and zero traction boundary conditions such as deflection of a cantilever beam under its own weight, the solution and the test spaces are both $H^{1}\left(T \mathcal{B}, \Gamma_{d}\right) \times H^{\mathbf{c}}\left(\mathcal{B}, \Gamma_{d}\right) \times H^{\mathbf{d}}\left(\mathcal{B}, \Gamma_{t}\right)$.

Remark 1. Let $\mathcal{D}$ be the space of $H^{1}$ vector fields satisfying the displacement boundary condition (2.3d) and let $\mathcal{A}$ be the space of $H^{\mathrm{d}}\left(\begin{array}{l}2 \\ 0\end{array}\right)$-tensor fields that satisfy the traction boundary condition (2.5). The weak formulation (2.8) corresponds to a saddle point of the Hu-Washizu-type functional $\mathrm{J}: \mathcal{D} \times H^{\mathbf{c}}(\mathcal{B}) \times \mathcal{A} \rightarrow \mathbb{R}$ defined as

$$
\mathrm{J}(\boldsymbol{\Upsilon}, \boldsymbol{\kappa}, \boldsymbol{\pi})=\int_{\mathcal{B}} \widetilde{W}(\boldsymbol{\kappa}) d A-\langle\langle\boldsymbol{\kappa}, \boldsymbol{\pi}\rangle\rangle_{L^{2}}+\left\langle\langle\boldsymbol{\pi}, \operatorname{grad} \mathbf{\Upsilon}\rangle_{L^{2}}-\left\langle\left\langle\rho_{0} \boldsymbol{B}, \mathbf{\Upsilon}\right\rangle_{L^{2}}-\int_{\Gamma_{t}}\langle\overline{\boldsymbol{T}}, \mathbf{\Upsilon}\rangle d s\right.\right.
$$

where $\widetilde{W}$ is the stored energy function expressed as a function of the displacement gradient. To see this, let $(\boldsymbol{U}, \boldsymbol{K}, \boldsymbol{P}) \in \mathcal{D} \times H^{\mathbf{c}}(\mathcal{B}) \times \mathcal{A}$ and note that any element of $\mathcal{D} \times H^{\mathbf{c}}(\mathcal{B}) \times \mathcal{A}$ can be written as $(\boldsymbol{U}+\alpha \boldsymbol{\Upsilon}, \boldsymbol{K}+\beta \boldsymbol{\kappa}, \boldsymbol{P}+\gamma \boldsymbol{\pi})$, where $\alpha, \beta, \gamma \in \mathbb{R}$ and $(\boldsymbol{\Upsilon}, \boldsymbol{\kappa}, \boldsymbol{\pi}) \in H^{1}\left(T \mathcal{B}, \Gamma_{d}\right) \times H^{\mathbf{c}}(\mathcal{B}) \times H^{\mathrm{d}}\left(\mathcal{B}, \Gamma_{t}\right)$. Next, let

$$
\mathrm{I}(\alpha, \beta, \gamma):=\mathrm{J}(\boldsymbol{U}+\alpha \boldsymbol{\Upsilon}, \boldsymbol{K}+\beta \boldsymbol{\kappa}, \boldsymbol{P}+\gamma \boldsymbol{\pi}) .
$$

It is straightforward to show that $\frac{\partial \mathrm{I}}{\partial \alpha}, \frac{\partial \mathrm{I}}{\partial \beta}$, and $\frac{\partial \mathrm{I}}{\partial \gamma}$ are zero at $(\alpha, \beta, \gamma)=(0,0,0)$ if and only if the relations (2.8) hold. The solution $(\boldsymbol{U}, \boldsymbol{K}, \boldsymbol{P})$ of (2.8) is a saddle point of the functional J, since the sign of $\left.\frac{\partial^{2} \mathrm{I}}{\partial \beta \partial \gamma}\right|_{(0,0,0)}=-\langle\langle\boldsymbol{\kappa}, \boldsymbol{\pi}\rangle\rangle_{L^{2}}$, depends on the way that one approaches $(\boldsymbol{U}, \boldsymbol{K}, \boldsymbol{P})$. Therefore, (2.8) is a mixed formulation for nonlinear elastostatics.

\section{$3 \quad$ Finite Element Approximations}

Our goal in this section is to write mixed finite element methods for approximating the mixed formulation (2.8) by using the Hilbert complexes introduced in $\S 2.1$. We assume that $\overline{\mathcal{B}}$ is a bounded polygonal domain with a triangulation $\mathcal{B}_{h}$, where $\mathcal{B}_{h}$ consists of arbitrary triangles $\mathcal{T}$, with

$$
h:=\max _{\mathcal{T} \in \mathcal{B}_{h}} \operatorname{diam} \mathcal{T},
$$


being the discretization parameter. The intersection of any two distinct triangles is either empty or a common edge/vertex of each triangle. Thus, $\overline{\mathcal{B}}$ is the polytope of the simplicial complex associated to $\mathcal{B}_{h}$. The set of all vertices and edges of $\mathcal{T}$ are denoted by $\Delta_{0}(\mathcal{T})$ and $\Delta_{1}(\mathcal{T})$, respectively. Similarly, $\Delta_{0}\left(\mathcal{B}_{h}\right), \Delta_{1}\left(\mathcal{B}_{h}\right)$, and $\Delta_{2}\left(\mathcal{B}_{h}\right)$ denote the sets of all vertices, edges, and triangles of $\mathcal{B}_{h}$. Let $\Delta_{0}^{\partial}\left(\mathcal{B}_{h}\right)$ and $\Delta_{1}^{\partial}\left(\mathcal{B}_{h}\right)$ be the sets of all vertices and edges of $\mathcal{B}_{h}$, which lie on $\partial \mathcal{B}$. The set of all interior edges are denoted by $\Delta_{1}^{\mathrm{i}}\left(\mathcal{B}_{h}\right):=\Delta_{1}\left(\mathcal{B}_{h}\right) \backslash \Delta_{1}^{\partial}\left(\mathcal{B}_{h}\right)$. We assume that $\mathcal{B}_{h}$ is compatible with $\Gamma_{d}$ and $\Gamma_{t}$ in the sense that $\Delta_{1}^{\partial}\left(\mathcal{B}_{h}\right)=\mathcal{T}_{d}^{h} \cup \mathcal{T}_{t}^{h}$, where the disjoint sets $\mathcal{T}_{d}^{h}$ and $\mathcal{T}_{t}^{h}$ are meshes for $\Gamma_{d}$ and $\Gamma_{t}$.

\subsection{Finite Element Spaces and Discrete Hilbert Complexes}

Next, we use appropriate piecewise polynomial vector and tensor fields on $\mathcal{B}_{h}$ for discretizing the Hilbert complexes (2.1) and (2.2) and define suitable finite element spaces for discretizing (2.8). We begin our discussion by introducing some polynomial fields in $\mathbb{R}^{2}$, which will be used for defining the piecewise polynomial fields.

\subsubsection{Polynomial Tensor Fields}

Let $\mathcal{P}_{r}\left(\mathbb{R}^{2}\right)$ be the space of $\mathbb{R}$-valued polynomials in two variables $\left\{\mathrm{X}^{1}, \mathrm{X}^{2}\right\}$ of degree at most $r \geq 0$ and let $\mathcal{H}_{r}\left(\mathbb{R}^{2}\right) \subset \mathcal{P}_{r}\left(\mathbb{R}^{2}\right)$ be the space of homogeneous polynomials of degree $r$, i.e. all terms of members of $\mathcal{H}_{r}\left(\mathbb{R}^{2}\right)$ are of degree $r$. These spaces are assumed to be the zero space if $r<0$. The spaces of polynomial vector and $\left(\begin{array}{l}2 \\ 0\end{array}\right)$-tensor fields in $\mathbb{R}^{2}$ with Cartesian components in $\mathcal{P}_{r}\left(\mathbb{R}^{2}\right)$ are denoted by $\mathcal{P}_{r}\left(T \mathbb{R}^{2}\right)$ and $\mathcal{P}_{r}\left(\otimes^{2} T \mathbb{R}^{2}\right)$, respectively. The spaces of homogeneous polynomial fields $\mathcal{H}_{r}\left(T \mathbb{R}^{2}\right)$ and $\mathcal{H}_{r}\left(\otimes^{2} T \mathbb{R}^{2}\right)$ are defined analogously. It is straightforward to show that

$$
\begin{aligned}
& \operatorname{dim} \mathcal{P}_{r}\left(\otimes^{2} T \mathbb{R}^{2}\right)=2 \operatorname{dim} \mathcal{P}_{r}\left(T \mathbb{R}^{2}\right)=2(r+1)(r+2), \\
& \operatorname{dim} \mathcal{H}_{r}\left(\otimes^{2} T \mathbb{R}^{2}\right)=2 \operatorname{dim} \mathcal{H}_{r}\left(T \mathbb{R}^{2}\right)=4(r+1) .
\end{aligned}
$$

One can use $\mathcal{P}_{r}$ fields to write the following polynomial complexes

$$
\begin{aligned}
& 0 \longrightarrow \mathcal{P}_{r+2}\left(T \mathbb{R}^{2}\right) \stackrel{\text { grad }}{\longrightarrow} \mathcal{P}_{r+1}\left(\otimes^{2} T \mathbb{R}^{2}\right) \stackrel{\text { c }}{\longrightarrow} \mathcal{P}_{r}\left(T \mathbb{R}^{2}\right) \longrightarrow 0 \\
& 0 \longrightarrow \mathcal{P}_{r+2}\left(T \mathbb{R}^{2}\right) \stackrel{\text { s }}{\longrightarrow} \mathcal{P}_{r+1}\left(\otimes^{2} T \mathbb{R}^{2}\right) \stackrel{\text { div }}{\longrightarrow} \mathcal{P}_{r}\left(T \mathbb{R}^{2}\right) \longrightarrow 0
\end{aligned}
$$

Next, we introduce two subspaces of $\mathcal{P}_{r}\left(\otimes^{2} T \mathbb{R}^{2}\right)$. Suppose $Y^{I}\left(\mathrm{X}^{1}, \mathrm{X}^{2}\right)$ and $T^{I J}\left(\mathrm{X}^{1}, \mathrm{X}^{2}\right), I, J=1,2$, are the Cartesian components of a vector field $\boldsymbol{Y}$ and a $\left(\begin{array}{l}2 \\ 0\end{array}\right)$-tensor field $\boldsymbol{T}$. We define the operators $\mathbf{K}_{1}, \widetilde{\mathbf{K}}_{1}: \mathcal{P}_{r}\left(\otimes^{2} T \mathbb{R}^{2}\right) \rightarrow \mathcal{P}_{r+1}\left(T \mathbb{R}^{2}\right)$ and $\mathbf{K}_{2}, \widetilde{\mathbf{K}}_{2}: \mathcal{P}_{r}\left(T \mathbb{R}^{2}\right) \rightarrow \mathcal{P}_{r+1}\left(\otimes^{2} T \mathbb{R}^{2}\right)$ as

$$
\begin{aligned}
\mathbf{K}_{1}(\boldsymbol{T}):=\left[\begin{array}{ll}
\mathrm{X}^{1} T^{11}+\mathrm{X}^{2} T^{12} \\
\mathrm{X}^{1} T^{21}+\mathrm{X}^{2} T^{22}
\end{array}\right], & \widetilde{\mathbf{K}}_{1}(\boldsymbol{T}):=\left[\begin{array}{ll}
-\mathrm{X}^{1} T^{12}+\mathrm{X}^{2} T^{11} \\
-\mathrm{X}^{1} T^{22}+\mathrm{X}^{2} T^{21}
\end{array}\right], \\
\mathbf{K}_{2}(\boldsymbol{Y}):=\left[\begin{array}{ll}
-\mathrm{X}^{2} Y^{1} & \mathrm{X}^{1} Y^{1} \\
-\mathrm{X}^{2} Y^{2} & \mathrm{X}^{1} Y^{2}
\end{array}\right], & \widetilde{\mathbf{K}}_{2}(\boldsymbol{Y}):=\left[\begin{array}{ll}
\mathrm{X}^{1} Y^{1} & \mathrm{X}^{2} Y^{1} \\
\mathrm{X}^{1} Y^{2} & \mathrm{X}^{2} Y^{2}
\end{array}\right] .
\end{aligned}
$$

It is straightforward to show that $\mathbf{K}_{1} \circ \mathbf{K}_{2}=\mathbf{0}$, and $\widetilde{\mathbf{K}}_{1} \circ \widetilde{\mathbf{K}}_{2}=\mathbf{0}$, and therefore, these operators give rise to the following polynomial complexes

$$
\begin{aligned}
& 0 \longrightarrow \mathcal{P}_{r}\left(T \mathbb{R}^{2}\right) \stackrel{\mathbf{K}_{2}}{\longrightarrow} \mathcal{P}_{r+1}\left(\otimes^{2} T \mathbb{R}^{2}\right) \stackrel{\mathbf{K}_{1}}{\longrightarrow} \mathcal{P}_{r+2}\left(T \mathbb{R}^{2}\right) \longrightarrow 0, \\
& 0 \longrightarrow \mathcal{P}_{r}\left(T \mathbb{R}^{2}\right) \stackrel{\widetilde{\mathbf{K}}_{2}}{\longrightarrow} \mathcal{P}_{r+1}\left(\otimes^{2} T \mathbb{R}^{2}\right) \stackrel{\widetilde{\mathbf{K}}_{1}}{\longrightarrow} \mathcal{P}_{r+2}\left(T \mathbb{R}^{2}\right) \longrightarrow 0
\end{aligned}
$$


It turns out that any $\boldsymbol{T} \in \mathcal{H}_{r}\left(\otimes^{2} T \mathbb{R}^{2}\right)$ can be decomposed as

$$
\boldsymbol{T}=\mathbf{K}_{2}\left(\boldsymbol{W}_{\boldsymbol{T}}\right)+\operatorname{grad}\left(\boldsymbol{Y}_{\boldsymbol{T}}\right)=\widetilde{\mathbf{K}}_{2}\left(\widetilde{\boldsymbol{W}}_{\boldsymbol{T}}\right)+\mathbf{s}\left(\widetilde{\boldsymbol{Y}}_{\boldsymbol{T}}\right),
$$

where $\boldsymbol{W}_{\boldsymbol{T}}, \widetilde{\boldsymbol{W}}_{\boldsymbol{T}} \in \mathcal{H}_{r-1}\left(T \mathbb{R}^{2}\right)$ and $\boldsymbol{Y}_{\boldsymbol{T}}, \widetilde{\boldsymbol{Y}}_{\boldsymbol{T}} \in \mathcal{H}_{r+1}\left(T \mathbb{R}^{2}\right)$. This result can be stated as follows.

Theorem 2. The following decompositions hold:

$$
\begin{aligned}
& \mathcal{H}_{r}\left(\otimes^{2} T \mathbb{R}^{2}\right)=\mathbf{K}_{2}\left(\mathcal{H}_{r-1}\left(T \mathbb{R}^{2}\right)\right) \oplus \operatorname{grad}\left(\mathcal{H}_{r+1}\left(T \mathbb{R}^{2}\right)\right) \\
& \mathcal{H}_{r}\left(\otimes^{2} T \mathbb{R}^{2}\right)=\widetilde{\mathbf{K}}_{2}\left(\mathcal{H}_{r-1}\left(T \mathbb{R}^{2}\right)\right) \oplus \mathbf{s}\left(\mathcal{H}_{r+1}\left(T \mathbb{R}^{2}\right)\right)
\end{aligned}
$$

where

$$
\begin{aligned}
& \operatorname{dim} \mathbf{K}_{2}\left(\mathcal{H}_{r-1}\left(T \mathbb{R}^{2}\right)\right)=\operatorname{dim} \widetilde{\mathbf{K}}_{2}\left(\mathcal{H}_{r-1}\left(T \mathbb{R}^{2}\right)\right)=2 r, \\
& \operatorname{dim} \operatorname{grad}\left(\mathcal{H}_{r+1}\left(T \mathbb{R}^{2}\right)\right)=\operatorname{dim} \mathbf{s}\left(\mathcal{H}_{r+1}\left(T \mathbb{R}^{2}\right)\right)=2(r+2) .
\end{aligned}
$$

Proof. As discussed in $[32, \S 3.1]$, there is an isomorphism between the Hilbert complexes (2.1) and (2.2) and the de Rham complex. One can show that this isomorphism induces an isomorphism between the complexes (3.2) and (3.3) and a standard complex in differential geometry called the Koszul complex (for more details on this complex see $[30, \S 5.1 .2]$ or $[38, \S 3.4 .6]$ ). Consequently, the above decompositions directly follow from the decomposition of polynomial 1-forms induced by the exterior derivative and the Koszul differential, which is introduced by Arnold et al. [29, page 32].

Since $\mathcal{P}_{r}\left(\otimes^{2} T \mathbb{R}^{2}\right)=\mathcal{P}_{r-1}\left(\otimes^{2} T \mathbb{R}^{2}\right) \oplus \mathcal{H}_{r}\left(\otimes^{2} T \mathbb{R}^{2}\right)$, the decompositions introduced in the above theorem allow one to define the following subspaces of $\mathcal{P}_{r}\left(\otimes^{2} T \mathbb{R}^{n}\right)$ :

$$
\begin{aligned}
& \mathcal{P}_{r}^{-}\left(\otimes^{2} T \mathbb{R}^{2}\right):=\mathcal{P}_{r-1}\left(\otimes^{2} T \mathbb{R}^{2}\right) \oplus \mathbf{K}_{2}\left(\mathcal{H}_{r-1}\left(T \mathbb{R}^{2}\right)\right), \\
& \mathcal{P}_{r}^{\ominus}\left(\otimes^{2} T \mathbb{R}^{2}\right):=\mathcal{P}_{r-1}\left(\otimes^{2} T \mathbb{R}^{2}\right) \oplus \widetilde{\mathbf{K}}_{2}\left(\mathcal{H}_{r-1}\left(T \mathbb{R}^{2}\right)\right),
\end{aligned}
$$

where $\operatorname{dim} \mathcal{P}_{r}^{-}\left(\otimes^{2} T \mathbb{R}^{2}\right)=\operatorname{dim} \mathcal{P}_{r}^{\ominus}\left(\otimes^{2} T \mathbb{R}^{2}\right)=2 r(r+2)$.

Example 3. Let $(X, Y)=\left(\mathrm{X}^{1}, \mathrm{X}^{2}\right)$. For $r=1$, the 12 -dimensional space $\mathcal{P}_{1}\left(\otimes^{2} T \mathbb{R}^{2}\right)$ can be written as

$$
\mathcal{P}_{1}\left(\otimes^{2} T \mathbb{R}^{2}\right)=\left\{\mathbf{A}_{0}+X \cdot \mathbf{A}_{1}+Y \cdot \mathbf{A}_{2}, \forall \mathbf{A}_{i} \in \mathbb{R}^{2 \times 2}\right\}
$$

where $\mathbb{R}^{2 \times 2}$ is the space of $2 \times 2$ matrices. The subspaces $\mathcal{P}_{1}^{-}\left(\otimes^{2} T \mathbb{R}^{2}\right)$ and $\mathcal{P}_{1}^{\ominus}\left(\otimes^{2} T \mathbb{R}^{2}\right)$ are 6 dimensional and are given by

$$
\begin{aligned}
& \mathcal{P}_{1}^{-}\left(\otimes^{2} T \mathbb{R}^{2}\right)=\left\{\mathbf{A}+\left[\begin{array}{ll}
-a Y & a X \\
-b Y & b X
\end{array}\right], \forall a, b \in \mathbb{R}, \forall \mathbf{A} \in \mathbb{R}^{2 \times 2}\right\}, \\
& \mathcal{P}_{1}^{\ominus}\left(\otimes^{2} T \mathbb{R}^{2}\right)=\left\{\mathbf{A}+\left[\begin{array}{ll}
a X & a Y \\
b X & b Y
\end{array}\right], \forall a, b \in \mathbb{R}, \forall \mathbf{A} \in \mathbb{R}^{2 \times 2}\right\} .
\end{aligned}
$$

For $r=2$, we have $\operatorname{dim} \mathcal{P}_{2}\left(\otimes^{2} T \mathbb{R}^{2}\right)=24$, and $\operatorname{dim} \mathcal{P}_{2}^{-}\left(\otimes^{2} T \mathbb{R}^{2}\right)=\operatorname{dim} \mathcal{P}_{2}^{\ominus}\left(\otimes^{2} T \mathbb{R}^{2}\right)=16$, where

$$
\begin{aligned}
\mathcal{P}_{2}\left(\otimes^{2} T \mathbb{R}^{2}\right)= & \left\{\mathbf{A}_{0}+X \cdot \mathbf{A}_{1}+Y \cdot \mathbf{A}_{2}+X^{2} \cdot \mathbf{A}_{3}+X Y \cdot \mathbf{A}_{4}+Y^{2} \cdot \mathbf{A}_{5}, \forall \mathbf{A}_{i} \in \mathbb{R}^{2 \times 2}\right\}, \\
\mathcal{P}_{2}^{-}\left(\otimes^{2} T \mathbb{R}^{2}\right)= & \left\{\mathbf{A}_{0}+X \cdot \mathbf{A}_{1}+Y \cdot \mathbf{A}_{2}\right. \\
& \left.+\left[\begin{array}{ll}
-a_{1} X Y-a_{2} Y^{2} & a_{1} X^{2}+a_{2} X Y \\
-b_{1} X Y-b_{2} Y^{2} & b_{1} X^{2}+b_{2} X Y
\end{array}\right], \forall a_{i}, b_{j} \in \mathbb{R}, \forall \mathbf{A}_{k} \in \mathbb{R}^{2 \times 2}\right\},
\end{aligned}
$$




$$
\begin{aligned}
& \mathcal{P}_{2}^{\ominus}\left(\otimes^{2} T \mathbb{R}^{2}\right)=\left\{\mathbf{A}_{0}+X \cdot \mathbf{A}_{1}+Y \cdot \mathbf{A}_{2}\right. \\
& \left.+\left[\begin{array}{ll}
a_{1} X^{2}+a_{2} X Y & a_{1} X Y+a_{2} Y^{2} \\
b_{1} X^{2}+b_{2} X Y & b_{1} X Y+b_{2} Y^{2}
\end{array}\right], \forall a_{i}, b_{j} \in \mathbb{R}, \forall \mathbf{A}_{k} \in \mathbb{R}^{2 \times 2}\right\} .
\end{aligned}
$$

The subspaces defined in (3.4) give rise to the following polynomial complexes:

$$
\begin{aligned}
& 0 \longrightarrow \mathcal{P}_{r+1}\left(T \mathbb{R}^{2}\right) \stackrel{\text { grad }}{\longrightarrow} \mathcal{P}_{r+1}^{-}\left(\otimes^{2} T \mathbb{R}^{2}\right) \stackrel{\text { c }}{\longrightarrow} \mathcal{P}_{r}\left(T \mathbb{R}^{2}\right) \longrightarrow 0 \\
& 0 \longrightarrow \mathcal{P}_{r+1}\left(T \mathbb{R}^{2}\right) \stackrel{\text { s }}{\longrightarrow} \mathcal{P}_{r+1}^{\ominus}\left(\otimes^{2} T \mathbb{R}^{2}\right) \stackrel{- \text { div }}{\longrightarrow} \mathcal{P}_{r}\left(T \mathbb{R}^{2}\right) \longrightarrow 0
\end{aligned}
$$

Remark 4. By using a subspace of the space of polynomial 1-forms introduced in [29, §3.3], one can write the following subspaces of $\mathcal{P}_{r}\left(T \mathbb{R}^{2}\right)$ :

$$
\begin{aligned}
& \mathcal{P}_{r}^{-}\left(T \mathbb{R}^{2}\right):=\mathcal{P}_{r-1}\left(T \mathbb{R}^{2}\right) \oplus \mathrm{K}_{2}\left(\mathcal{H}_{r-1}\left(\mathbb{R}^{2}\right)\right), \\
& \mathcal{P}_{r}^{\ominus}\left(T \mathbb{R}^{2}\right):=\mathcal{P}_{r-1}\left(T \mathbb{R}^{2}\right) \oplus \widetilde{\mathrm{K}}_{2}\left(\mathcal{H}_{r-1}\left(\mathbb{R}^{2}\right)\right),
\end{aligned}
$$

where

$$
\mathrm{K}_{2}(f):=\left[\begin{array}{c}
-\mathrm{X}^{2} f \\
\mathrm{X}^{1} f
\end{array}\right], \quad \widetilde{\mathrm{K}}_{2}(f):=\left[\begin{array}{l}
\mathrm{X}^{1} f \\
\mathrm{X}^{2} f
\end{array}\right] .
$$

Clearly, the spaces defined in (3.4) are the tensorial analogues of (3.6) that are obtained by identifying $\left(\begin{array}{l}2 \\ 0\end{array}\right)$-tensors with the two vector fields associated to their rows.

\subsubsection{Finite Element Spaces}

We are now ready to introduce appropriate finite element spaces for discretizing (2.8). Suppose $\mathcal{P}_{r}\left(T \mathcal{B}_{h}\right)$ is the space of piecewise polynomial vector fields of order $r$ on the triangulation $\mathcal{B}_{h}$ and let $\mathcal{P}_{r}^{1}\left(T \mathcal{B}_{h}\right)$ be the standard Lagrange finite element space of vector fields of degree $r$ on $\mathcal{B}_{h}$, that is, $\mathcal{P}_{r}^{1}\left(T \mathcal{B}_{h}\right)$ is the space of piecewise polynomial vector fields of degree $r$, which are continuous along all edges. Also let $\mathcal{P}_{r}^{1}\left(T \mathcal{B}_{h}, \Gamma_{d}\right):=\left\{\boldsymbol{Y} \in \mathcal{P}_{r}^{1}\left(T \mathcal{B}_{h}\right):\left.\boldsymbol{Y}\right|_{\Gamma_{d}}=\mathbf{0}\right\}$. Moreover, suppose $\mathcal{P}_{r}\left(\otimes^{2} T \mathcal{B}_{h}\right)$ is the space of piecewise polynomial $\left(\begin{array}{l}2 \\ 0\end{array}\right)$-tensor fields of order $r$ on $\mathcal{B}_{h}$ and let $\mathcal{P}_{r}^{-}\left(\otimes^{2} T \mathcal{B}_{h}\right)\left(\mathcal{P}_{r}^{\ominus}\left(\otimes^{2} T \mathcal{B}_{h}\right)\right)$ be the space of all $\boldsymbol{T} \in \mathcal{P}_{r}\left(\otimes^{2} T \mathcal{B}_{h}\right)$ such that $\left.\boldsymbol{T}\right|_{\mathcal{T}}, \forall \mathcal{T} \in \mathcal{B}_{h}$, is a $\mathcal{P}_{r}^{-}\left(\mathcal{P}_{r}^{\ominus}\right)$ polynomial.

In order to define suitable $H^{\mathbf{c}}$ and $H^{\mathbf{d}}$-conformal finite element spaces, we use the following notions of the jump of tangent and normal tractions of a $\left(\begin{array}{l}2 \\ 0\end{array}\right)$-tensor across an edge. Let $\mathcal{E} \in \Delta_{1}^{\mathrm{i}}\left(\mathcal{B}_{h}\right)$ be a common edge of $\mathcal{T}, \mathcal{T}^{\prime} \in \mathcal{B}_{h}$ and let $\mathbf{t}(\mathbf{n})$ be a unit vector parallel (normal) to $\mathcal{E}$. The jump of the tangent traction of $\boldsymbol{T} \in \mathcal{P}_{r}\left(\otimes^{2} T \mathbb{R}^{2}\right)$ across $\mathcal{E}$ is defined as

$$
\llbracket \mathrm{t} \boldsymbol{T} \rrbracket_{\mathcal{E}}:=\boldsymbol{T}_{\mathcal{T}}(\mathbf{t})-\boldsymbol{T}_{\mathcal{T}^{\prime}}(\mathbf{t}),
$$

where $\boldsymbol{T}_{\mathcal{T}}:=\left.\boldsymbol{T}\right|_{\mathcal{T}}$. Similarly, the jump of the normal traction of $\boldsymbol{T}$ across $\mathcal{E}$ is defined as

$$
\llbracket \mathrm{n} \boldsymbol{T} \rrbracket_{\mathcal{E}}:=\boldsymbol{T}_{\mathcal{T}}(\mathbf{n})-\boldsymbol{T}_{\mathcal{T}^{\prime}}(\mathbf{n}) .
$$

Next, we define the following spaces of piecewise polynomial $\left(\begin{array}{l}2 \\ 0\end{array}\right)$-tensors with zero tangent traction jumps:

$$
\begin{aligned}
& \mathcal{P}_{r}^{\mathbf{c}}\left(\mathcal{B}_{h}\right):=\left\{\boldsymbol{T} \in \mathcal{P}_{r}\left(\otimes^{2} T \mathcal{B}_{h}\right): \llbracket \mathrm{t} \boldsymbol{T} \rrbracket_{\mathcal{E}}=0, \forall \mathcal{E} \in \Delta_{1}^{\mathrm{i}}\left(\mathcal{B}_{h}\right)\right\}, \\
& \mathcal{P}_{r}^{\mathbf{c}-}\left(\mathcal{B}_{h}\right):=\left\{\boldsymbol{T} \in \mathcal{P}_{r}^{-}\left(\otimes^{2} T \mathcal{B}_{h}\right): \llbracket \mathrm{t} \boldsymbol{T} \rrbracket_{\mathcal{E}}=0, \forall \mathcal{E} \in \Delta_{1}^{\mathrm{i}}\left(\mathcal{B}_{h}\right)\right\} .
\end{aligned}
$$

By $\mathcal{P}_{r}^{\mathbf{c}}\left(\mathcal{B}_{h}, \Gamma_{d}\right)\left(\mathcal{P}_{r}^{\mathbf{c}-}\left(\mathcal{B}_{h}, \Gamma_{d}\right)\right)$ we denote the space of $\mathcal{P}_{r}^{\mathbf{c}}\left(\mathcal{P}_{r}^{\mathbf{c}-}\right)$ tensors, which are normal to $\Gamma_{d}$ (see 
$\S 2.1)$. Similarly, one can define the following spaces

$$
\begin{aligned}
& \mathcal{P}_{r}^{\mathbf{d}}\left(\mathcal{B}_{h}\right):=\left\{\boldsymbol{T} \in \mathcal{P}_{r}\left(\otimes^{2} T \mathcal{B}_{h}\right): \llbracket \mathrm{n} \boldsymbol{T} \rrbracket \mathcal{E}=0, \forall \mathcal{E} \in \Delta_{1}^{\mathrm{i}}\left(\mathcal{B}_{h}\right)\right\}, \\
& \mathcal{P}_{r}^{\mathbf{d}-}\left(\mathcal{B}_{h}\right):=\left\{\boldsymbol{T} \in \mathcal{P}_{r}^{\ominus}\left(\otimes^{2} T \mathcal{B}_{h}\right): \llbracket \mathrm{n} \boldsymbol{T} \rrbracket \mathcal{E}=0, \forall \mathcal{E} \in \Delta_{1}^{\mathrm{i}}\left(\mathcal{B}_{h}\right)\right\} .
\end{aligned}
$$

The space of $\mathcal{P}_{r}^{\mathbf{d}}\left(\mathcal{P}_{r}^{\mathbf{d}-}\right)$ tensors with zero traction on $\Gamma_{t}$ is denoted by $\mathcal{P}_{r}^{\mathbf{d}}\left(\mathcal{B}_{h}, \Gamma_{t}\right)\left(\mathcal{P}_{r}^{\mathbf{d}-}\left(\mathcal{B}_{h}, \Gamma_{t}\right)\right)$.

Remark 5. Suppose $\boldsymbol{K} \in \mathcal{P}_{r}\left(\otimes^{2} T \mathcal{B}_{h}\right)$ represents a displacement gradient on $\mathcal{B}_{h}$. Then, if we consider the internal edges as interfaces, the zero jump condition in (3.7) is simply the Hadamard jump condition for the strain compatibility along edges, e.g. see [39, page 14]. The Hadamard jump condition is a necessary compatibility condition for the existence of an $H^{1}$-displacement field with the displacement gradient $\boldsymbol{K} .^{2}$ On the other hand, if $\boldsymbol{P} \in \mathcal{P}_{r}\left(\otimes^{2} T \mathcal{B}_{h}\right)$ represents a stress tensor, the zero jump condition in (3.8) implies that traction vectors of $\boldsymbol{P}$ along all the internal interfaces are opposite to each other.

The above remark provides a physical justification for the following result.

Theorem 6. One has $\mathcal{P}_{r}^{\mathbf{c}}\left(\mathcal{B}_{h}\right), \mathcal{P}_{r}^{\mathbf{c}-}\left(\mathcal{B}_{h}\right) \subset H^{\mathbf{c}}(\mathcal{B})$ and $\mathcal{P}_{r}^{\mathbf{d}}\left(\mathcal{B}_{h}\right), \mathcal{P}_{r}^{\mathbf{d}-}\left(\mathcal{B}_{h}\right) \subset H^{\mathbf{d}}(\mathcal{B})$

Proof. These results are direct consequences of the definition of the operators c and div (in the weak sense) together with Green's formula (2.4) and the following Green's formula:

$$
\left\langle\langle\mathbf{c}(\boldsymbol{T}), \boldsymbol{Y}\rangle_{L^{2}}=\langle\langle\boldsymbol{T}, \mathbf{s}(\boldsymbol{Y})\rangle\rangle_{L^{2}}+\int_{\partial \mathcal{B}}\left\langle\boldsymbol{T}\left(\boldsymbol{t}_{\partial}\right), \boldsymbol{Y}\right\rangle\right\rangle d s
$$

where $\boldsymbol{t}_{\partial}$ is the oriented unit tangent vector field of $\partial \mathcal{B}$. Since the steps of the proof are similar to those for proving the $H^{1}$-conformity of Lagrange finite element spaces (e.g. see [26, Proposition 1.74]), we do not present the details of the proof here.

Our next goal is to show that piecewise polynomial spaces defined in (3.7) and (3.8) are actually finite element spaces, i.e. they can be generated through the finite element assembly process by using appropriate finite elements. Recall that a finite element on $\mathcal{T} \in \mathcal{B}_{h}$ is a triplet $(\mathcal{T}, \mathrm{P}, \Sigma)$, where $\mathrm{P}$ is an $m_{\mathrm{sh}}$-dimensional linear space of some polynomial functions on $\mathcal{T}$ and $\Sigma=\left\{\varsigma_{1}, \ldots, \varsigma_{m_{\mathrm{sh}}}\right\}$ is the set of local degrees of freedom [41]. We need to determine local degrees of freedom that can impose the zero jump conditions of (3.7) and (3.8). Let $\mathcal{P}_{r}\left(\otimes^{2} T \mathcal{T}\right), \mathcal{P}_{r}^{-}\left(\otimes^{2} T \mathcal{T}\right)$, and $\mathcal{P}_{r}^{\ominus}\left(\otimes^{2} T \mathcal{T}\right)$ be, respectively, the spaces of $\mathcal{P}_{r}, \mathcal{P}_{r}^{-}$, and $\mathcal{P}_{r}^{\ominus}$ polynomial $\left(\begin{array}{l}2 \\ 0\end{array}\right)$-tensors on $\mathcal{T}$ and suppose that $\mathcal{P}_{r}\left(\otimes^{2} T \mathcal{T}\right)^{*}, \mathcal{P}_{r}^{-}\left(\otimes^{2} T \mathcal{T}\right)^{*}$, and $\mathcal{P}_{r}^{\ominus}\left(\otimes^{2} T \mathcal{T}\right)^{*}$ are their dual spaces. It turns out that the following geometric decompositions of these dual spaces determine the interelement continuities of (3.7) and (3.8), in the sense that any dual basis $\Sigma$, which is consistent with these decompositions will impose the corresponding zero jump condition.

Theorem 7. Let $r>0$ be an integer and suppose the vector field $\overrightarrow{\boldsymbol{T}}_{I}:=T^{I J} \mathbf{E}_{J}, I=1,2$, is the $I$-th row of a $\left(\begin{array}{l}2 \\ 0\end{array}\right)$-tensor $\boldsymbol{T}$. Also for any $\mathcal{T} \in \mathcal{B}_{h}$ and each $\mathcal{E} \in \Delta_{1}(\mathcal{T})$, suppose that $\mathbf{t}_{\mathcal{E}}$ is the oriented unit tangent vector of $\mathcal{E}$ and $\mathbf{n}_{\mathcal{E}}$ is the unit outward vector normal to $\mathcal{E}$. Consider the following spaces:

$$
\begin{aligned}
& \left.W_{I, r}^{\mathbf{c}}(\mathcal{T}, \mathcal{E}):=\left\{\phi \in \mathcal{P}_{r}\left(\otimes^{2} T \mathcal{T}\right)^{*}: \exists f \in \mathcal{P}_{r}\left(\mathbb{R}^{2}\right) \text { such that } \phi(\boldsymbol{T})=\int_{\mathcal{E}} f\left\langle\overrightarrow{\boldsymbol{T}}_{I}, \mathbf{t}_{\mathcal{E}}\right\rangle\right\rangle d s\right\}, \\
& \left.W_{I, r}^{\mathbf{c}-}(\mathcal{T}, \mathcal{E}):=\left\{\phi \in \mathcal{P}_{r}^{-}\left(\otimes^{2} T \mathcal{T}\right)^{*}: \exists f \in \mathcal{P}_{r-1}\left(\mathbb{R}^{2}\right) \text { such that } \phi(\boldsymbol{T})=\int_{\mathcal{E}} f\left\langle\overrightarrow{\boldsymbol{T}}_{I}, \mathbf{t}_{\mathcal{E}}\right\rangle\right\rangle d s\right\}, \\
& W_{I, r}^{\mathbf{d}}(\mathcal{T}, \mathcal{E}):=\left\{\phi \in \mathcal{P}_{r}\left(\otimes^{2} T \mathcal{T}\right)^{*}: \exists f \in \mathcal{P}_{r}\left(\mathbb{R}^{2}\right) \text { such that } \phi(\boldsymbol{T})=\int_{\mathcal{E}} f\left\langle\overrightarrow{\boldsymbol{T}}_{I}, \mathbf{n}_{\mathcal{E}}\right\rangle d s\right\},
\end{aligned}
$$

\footnotetext{
${ }^{2}$ As discussed in [40], the Hadamard jump condition is not sufficient for the compatibility of displacement gradients if $\mathcal{B}_{h}$ is not simply-connected.
} 


$$
\begin{aligned}
W_{I, r}^{\mathbf{d}-}(\mathcal{T}, \mathcal{E}): & =\left\{\phi \in \mathcal{P}_{r}^{\ominus}\left(\otimes^{2} T \mathcal{T}\right)^{*}: \exists f \in \mathcal{P}_{r-1}\left(\mathbb{R}^{2}\right) \text { such that } \phi(\boldsymbol{T})=\int_{\mathcal{E}} f\left\langle\overrightarrow{\boldsymbol{T}}_{I}, \mathbf{n}_{\mathcal{E}}\right\rangle d s\right\}, \\
W_{I, r}^{\mathbf{c}}(\mathcal{T}, \mathcal{T}): & =\left\{\phi \in \mathcal{P}_{r}\left(\otimes^{2} T \mathcal{T}\right)^{*}: \exists \boldsymbol{Z} \in \mathcal{P}_{r-1}^{\ominus}\left(T \mathbb{R}^{2}\right) \text { such that } \phi(\boldsymbol{T})=\int_{\mathcal{T}}\left\langle\overrightarrow{\boldsymbol{T}}_{I}, \boldsymbol{Z}\right\rangle d A\right\}, \\
W_{I, r}^{\mathbf{c}-}(\mathcal{T}, \mathcal{T}): & =\left\{\phi \in \mathcal{P}_{r}^{-}\left(\otimes^{2} T \mathcal{T}\right)^{*}: \exists \boldsymbol{Z} \in \mathcal{P}_{r-2}\left(T \mathbb{R}^{2}\right) \text { such that } \phi(\boldsymbol{T})=\int_{\mathcal{T}}\left\langle\overrightarrow{\boldsymbol{T}}_{I}, \boldsymbol{Z}\right\rangle d A\right\}, \\
W_{I, r}^{\mathbf{d}}(\mathcal{T}, \mathcal{T}): & \left.=\left\{\phi \in \mathcal{P}_{r}\left(\otimes^{2} T \mathcal{T}\right)^{*}: \exists \boldsymbol{Z} \in \mathcal{P}_{r-1}^{-}\left(T \mathbb{R}^{2}\right) \text { such that } \phi(\boldsymbol{T})=\int_{\mathcal{T}}\left\langle\overrightarrow{\boldsymbol{T}}_{I}, \boldsymbol{Z}\right\rangle\right\rangle d A\right\}, \\
W_{I, r}^{\mathbf{d}-}(\mathcal{T}, \mathcal{T}): & =\left\{\phi \in \mathcal{P}_{r}^{\ominus}\left(\otimes^{2} T \mathcal{T}\right)^{*}: \exists \boldsymbol{Z} \in \mathcal{P}_{r-2}\left(T \mathbb{R}^{2}\right) \text { such that } \phi(\boldsymbol{T})=\int_{\mathcal{T}}\left\langle\left\langle\overrightarrow{\boldsymbol{T}}_{I}, \boldsymbol{Z}\right\rangle\right\rangle d A\right\},
\end{aligned}
$$

where

$$
\begin{aligned}
& \operatorname{dim} W_{I, r}^{\mathbf{c}}(\mathcal{T}, \mathcal{E})=\operatorname{dim} W_{I, r}^{\mathbf{d}}(\mathcal{T}, \mathcal{E})=r+1, \quad \operatorname{dim} W_{I, r}^{\mathbf{c}-}(\mathcal{T}, \mathcal{E})=\operatorname{dim} W_{I, r}^{\mathbf{d}-}(\mathcal{T}, \mathcal{E})=r, \\
& \operatorname{dim} W_{I, r}^{\mathbf{c}}(\mathcal{T}, \mathcal{T})=\operatorname{dim} W_{I, r}^{\mathbf{d}}(\mathcal{T}, \mathcal{T})=r^{2}-1, \quad \operatorname{dim} W_{I, r}^{\mathbf{c}-}(\mathcal{T}, \mathcal{T})=\operatorname{dim} W_{I, r}^{\mathbf{d}-}(\mathcal{T}, \mathcal{T})=r^{2}-r .
\end{aligned}
$$

Then, the following decompositions hold:

$$
\begin{aligned}
\mathcal{P}_{r}\left(\otimes^{2} T \mathcal{T}\right)^{*} & =\bigoplus_{\substack{\mathcal{E} \in \Delta_{1}(\mathcal{T}) \\
I=1,2}} W_{I, r}^{\mathbf{c}}(\mathcal{T}, \mathcal{E}) \oplus \bigoplus_{I=1,2} W_{I, r}^{\mathbf{c}}(\mathcal{T}, \mathcal{T}), \\
\mathcal{P}_{r}^{-}\left(\otimes^{2} T \mathcal{T}\right)^{*} & =\bigoplus_{\substack{\mathcal{E} \in \Delta_{1}(\mathcal{T}) \\
I=1,2}} W_{I, r}^{\mathbf{c}-}(\mathcal{T}, \mathcal{E}) \oplus \bigoplus_{I=1,2} W_{I, r}^{\mathbf{c}-}(\mathcal{T}, \mathcal{T}), \\
\mathcal{P}_{r}\left(\otimes^{2} T \mathcal{T}\right)^{*} & =\bigoplus_{\substack{\mathcal{E} \in \Delta_{1}(\mathcal{T}) \\
I=1,2}} W_{I, r}^{\mathbf{d}}(\mathcal{T}, \mathcal{E}) \oplus \bigoplus_{I=1,2} W_{I, r}^{\mathbf{d}}(\mathcal{T}, \mathcal{T}), \\
\mathcal{P}_{r}^{\ominus}\left(\otimes^{2} T \mathcal{T}\right)^{*}= & \bigoplus_{\substack{\mathcal{E} \in \Delta_{1}(\mathcal{T}) \\
I=1,2}} W_{I, r}^{\mathbf{d}-}(\mathcal{T}, \mathcal{E}) \oplus \bigoplus_{I=1,2} W_{I, r}^{\mathbf{d}-}(\mathcal{T}, \mathcal{T}) .
\end{aligned}
$$

Proof. The isomorphisms mentioned in the proof of Theorem 2 also induce isomorphisms between the dual spaces of polynomial tensors and the dual space of polynomial 1-forms and consequently, any decomposition for 1-forms induces a corresponding decomposition for tensors. In particular, the decompositions (3.9) directly follow from the geometric decomposition for polynomial differential forms introduced in [30, Theorem 5.5].

One can use the decompositions (3.9) to obtain the dual bases. For example, let us write a basis for $\mathcal{P}_{r}\left(\otimes^{2} T \mathcal{T}\right)^{*}$ that is consistent with (3.9a). For $I=1,2$, and any $\mathcal{E} \in \Delta_{1}(\mathcal{T})$, one chooses an arbitrary basis $\left\{f_{i}\right\}_{1 \leq i \leq r+1}$ for $\mathcal{P}_{r}\left(\mathbb{R}^{2}\right)$, such that $\left\{\phi_{I, i}^{\mathcal{T}, \mathcal{E}}\right\}_{1 \leq i \leq r+1}$ is a basis for $W_{I, r}^{\mathrm{c}}(\mathcal{T}, \mathcal{E})$, where $\phi_{I, i}^{\mathcal{T}, \mathcal{E}}(\boldsymbol{T})=\int_{\mathcal{E}} f_{i}\left\langle\left\langle\overrightarrow{\boldsymbol{T}}_{I}, \mathbf{t}_{\mathcal{E}}\right\rangle d s\right.$. Similarly, by choosing a basis $\left\{\boldsymbol{W}_{j}\right\}_{1 \leq j \leq r^{2}-1}$ for $\in \mathcal{P}_{r-1}^{\ominus}\left(T \mathbb{R}^{2}\right)$, one obtains a basis $\left\{\phi_{I, j}^{\mathcal{T}, \mathcal{T}}\right\}_{1 \leq j \leq r^{2}-1}$ for $W_{I, r}^{\mathbf{c}}(\mathcal{T}, \mathcal{T})$, with $\phi_{I, j}^{\mathcal{T}, \mathcal{T}}(\boldsymbol{T})=\int_{\mathcal{T}}\left\langle\left\langle\overrightarrow{\boldsymbol{T}}_{I}, \boldsymbol{W}_{j}\right\rangle d A\right.$. Then, one can write the following basis for $\mathcal{P}_{r}\left(\otimes^{2} T \mathcal{T}\right)^{*}$ :

$$
\Sigma^{\mathcal{T}, \mathbf{c}}:=\left\{\phi_{I, 1}^{\mathcal{T}, \mathcal{E}}, \ldots, \phi_{I, r+1}^{\mathcal{T}, \mathcal{E}}, \phi_{I, 1}^{\mathcal{T}, \mathcal{T}}, \ldots, \phi_{I, r^{2}-1}^{\mathcal{T}, \mathcal{T}}\right\}_{\substack{\mathcal{E} \in \Delta_{1}(\mathcal{T}) \\ I=1,2}}
$$


Table 1: Numbers of degrees of freedom (DOF) for tensorial finite elements.

\begin{tabular}{c|c|c|c}
\hline \multirow{2}{*}{ DOF } & \multicolumn{3}{|c}{ \# of DOF } \\
\cline { 2 - 4 } & total & for each edge & for $\mathcal{T}$ \\
\hline$\Sigma^{\mathcal{T}, \mathbf{c}}, \Sigma^{\mathcal{T}, \mathbf{d}}$ & $2(r+1)(r+2)$ & $2(\mathrm{r}+1)$ & $2\left(r^{2}-1\right)$ \\
$\Sigma^{\mathcal{T}, \mathbf{c}-}, \Sigma^{\mathcal{T}, \mathbf{d}-}$ & $2 r(r+2)$ & $2 \mathrm{r}$ & $2\left(r^{2}-r\right)$ \\
\hline
\end{tabular}

Note that by choosing different auxiliary polynomials, one obtains different bases for $\mathcal{P}_{r}\left(\otimes^{2} T \mathcal{T}\right)^{*}$, and therefore, bases consistent with (3.9a) are not unique. Similarly, one can obtain bases $\Sigma^{\mathcal{T}, \mathbf{c}-}, \Sigma^{\mathcal{T}, \mathbf{d}}$, and $\Sigma^{\mathcal{T}, \mathbf{d}-}$, which are consistent with $(3.9 \mathrm{~b}),(3.9 \mathrm{c})$, and $(3.9 \mathrm{~d})$, respectively.

Next, we consider the following finite elements:

$$
\begin{array}{ll}
\left(\mathcal{T}, \mathcal{P}_{r}\left(\otimes^{2} T \mathcal{T}\right), \Sigma^{\mathcal{T}, \mathbf{c}}\right), & \left(\mathcal{T}, \mathcal{P}_{r}^{-}\left(\otimes^{2} T \mathcal{T}\right), \Sigma^{\mathcal{T}, \mathbf{c}-}\right), \\
\left(\mathcal{T}, \mathcal{P}_{r}\left(\otimes^{2} T \mathcal{T}\right), \Sigma^{\mathcal{T}, \mathbf{d}}\right), & \left(\mathcal{T}, \mathcal{P}_{r}^{\ominus}\left(\otimes^{2} T \mathcal{T}\right), \Sigma^{\mathcal{T}, \mathbf{d}-}\right) .
\end{array}
$$

By choosing piecewise polynomial tensors that for degrees of freedom corresponding to the same edge, the same $I$, and the same auxiliary polynomial give the same values, one can construct the corresponding finite element spaces through the finite element assembly process. As a corollary to the discussions of $[29, \S 5.1]$, one concludes that the degrees of freedom of the above finite elements impose the interelement continuities of (3.7) and (3.8). The upshot is the following theorem.

Theorem 8. The spaces $\mathcal{P}_{r}^{\mathbf{c}}\left(\mathcal{B}_{h}\right)$ and $\mathcal{P}_{r}^{\mathbf{c}-}\left(\mathcal{B}_{h}\right)$ are generated by the finite elements $\left(\mathcal{T}, \mathcal{P}_{r}\left(\otimes^{2} T \mathcal{T}\right), \Sigma^{\mathcal{T}, \mathbf{c}}\right)$ and $\left(\mathcal{T}, \mathcal{P}_{r}^{-}\left(\otimes^{2} T \mathcal{T}\right), \Sigma^{\mathcal{T}, \mathbf{c}-}\right)$, respectively. Similarly, the spaces $\mathcal{P}_{r}^{\mathbf{d}}\left(\mathcal{B}_{h}\right)$ and $\mathcal{P}_{r}^{\mathbf{d}-}\left(\mathcal{B}_{h}\right)$ are generated by $\left(\mathcal{T}, \mathcal{P}_{r}\left(\otimes^{2} T \mathcal{T}\right), \Sigma^{\mathcal{T}, \mathbf{d}}\right)$ and $\left(\mathcal{T}, \mathcal{P}_{r}^{\ominus}\left(\otimes^{2} T \mathcal{T}\right), \Sigma^{\mathcal{T}, \mathbf{d}-}\right)$, respectively

As was mentioned earlier, bases consistent with the decompositions (3.9) are not unique. The above theorem suggests that the consistency of bases determines the interelement continuity rather than the specific choices of degrees of freedom. Table 1 depicts the numbers of local degrees of freedom of the finite elements (3.10).

Example 9. In the following, we write the local and global shape functions associated with the $H^{\mathbf{d}}$-conformal finite element spaces $\mathcal{P}_{r}^{\mathbf{d}}\left(\mathcal{B}_{h}\right)$ and $\mathcal{P}_{r}^{\mathbf{d}-}\left(\mathcal{B}_{h}\right)$, for $r=1,2$.

- $\mathcal{P}_{1}^{\mathbf{d}}\left(\mathcal{B}_{h}\right)$ : We have $\operatorname{dim} \mathcal{P}_{1}\left(\otimes^{2} T \mathcal{T}\right)=12$, and the dimensions of the components of the decomposition $(3.9 \mathrm{c})$ are $\operatorname{dim} W_{I, 1}^{\mathrm{d}}(\mathcal{T}, \mathcal{E})=2$ and $\operatorname{dim} W_{I, 1}^{\mathrm{d}}(\mathcal{T}, \mathcal{T})=0$. One can write the consistent basis

$$
\Sigma^{\mathcal{T}, \mathbf{d}}=\left\{\psi_{I, J}^{\mathcal{T}, \mathcal{E}}\right\}_{\substack{\mathcal{E} \in \Delta_{1}(\mathcal{T}) \\ I, J=1,2}}
$$

where

$$
\psi_{I, J}^{\mathcal{T}, \mathcal{E}}(\boldsymbol{T})=\int_{\mathcal{E}} s^{J-1}\left\langle\left\langle\overrightarrow{\boldsymbol{T}}_{I}, \mathbf{n}_{\mathcal{E}}\right\rangle d s\right.
$$

Local shape functions associated to $\Sigma^{\mathcal{T}, \mathbf{d}}$ are the members of the dual basis

$$
\left\{\boldsymbol{s}_{I, 1}^{\mathcal{T}, \mathcal{E}}, \boldsymbol{s}_{I, 2}^{\mathcal{T}, \mathcal{E}}\right\}_{\substack{\mathcal{E} \in \Delta_{1}(\mathcal{T}) \\ I=1,2}}
$$

which satisfy

$$
\psi_{K, L}^{\mathcal{T}, \mathcal{E}^{\prime}}\left(\boldsymbol{s}_{I, J}^{\mathcal{T}, \mathcal{E}}\right)= \begin{cases}1, & \text { if } \mathcal{E}=\mathcal{E}^{\prime} \text { and } I=K \text { and } J=L \\ 0, & \text { otherwise. }\end{cases}
$$




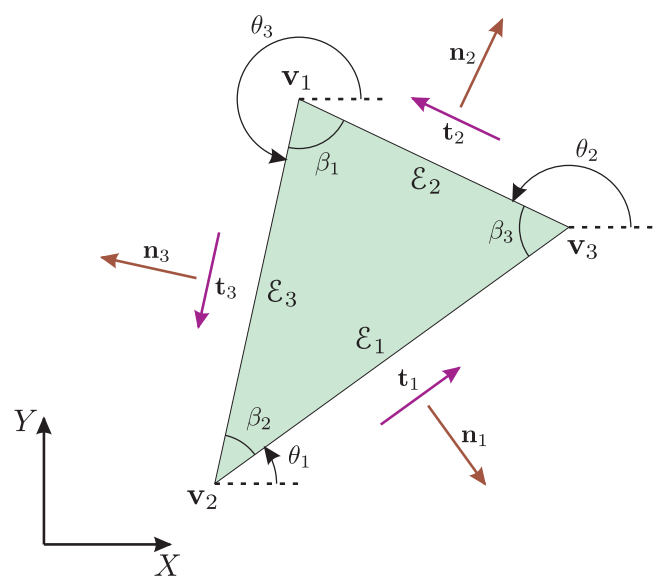

Figure 1: A triangle with vertices $\mathbf{v}_{i}$ and edges $\mathcal{E}_{i}, i=1,2,3$. The oriented unit tangent vector and the unit outward normal vector of $\mathcal{E}_{i}$ are denoted by $\mathbf{t}_{i}$ and $\mathbf{n}_{i}$, respectively.

For writing the explicit forms of the local shape functions, consider a triangle $\mathcal{T}$ with vertices $\mathbf{v}_{i}$ and edges $\varepsilon_{i}, i=1,2,3$, as shown in Figure 1 . Let $\ell_{i}$ and $A_{\mathcal{T}}$ denote the length of $\mathcal{E}_{i}$ and the area of $\mathcal{T}$, respectively. Also let $(X, Y)=\left(\mathrm{X}^{1}, \mathrm{X}^{2}\right)$ and suppose that $\left(v_{i}^{x}, v_{i}^{y}\right)$ are the Cartesian coordinates of the vertex $\mathbf{v}_{i}$. For each edge $\mathcal{E}_{i}$, there are four local shape functions $\boldsymbol{s}_{I, J}^{\mathcal{T}, \mathcal{E}_{i}}, I, J=1,2$. Using $(3.13)$, one can show that

$s_{1, J}^{\mathcal{T}, \mathcal{E}_{i}}(X, Y)=$

$\frac{1}{A_{\mathcal{T}}}\left[\begin{array}{cc}a_{X, J}^{\mathcal{T}, i} \cdot\left(X-v_{i+1}^{x}\right)+a_{Y, J}^{\mathcal{T}, i} \cdot\left(Y-v_{i+1}^{y}\right)+a_{J}^{\mathcal{T}, i} & b_{X, J}^{\mathcal{T}, i} \cdot\left(X-v_{i+1}^{x}\right)+b_{Y, J}^{\mathcal{T}, i} \cdot\left(Y-v_{i+1}^{y}\right)+b_{J}^{\mathcal{T}, i} \\ 0 & 0\end{array}\right]$,

with

$$
\begin{array}{llll}
a_{X, 1}^{\mathcal{T}, i}=\frac{1-\omega_{i}}{2}, & a_{Y, 1}^{\mathcal{T}, i}=\frac{3\left(-\cot \beta_{i}+\zeta_{i}\right)}{2}, & b_{X, 1}^{\mathcal{T}, i}=\frac{3\left(\cot \beta_{i}+\zeta_{i}\right)}{2}, & b_{Y, 1}^{\mathcal{T}, i}=\frac{1+\omega_{i}}{2}, \\
a_{X, 2}^{\mathcal{T}, i}=\frac{\omega_{i}}{\ell_{i}}, & a_{Y, 2}^{\mathcal{T}, i}=\frac{3\left(\cot \beta_{i}-\zeta_{i}\right)}{\ell_{i}}, & b_{X, 2}^{\mathcal{T}, i}=-\frac{3\left(\cot \beta_{i}+\zeta_{i}\right)}{\ell_{i}}, & b_{Y, 2}^{\mathcal{T}, i}=-\frac{\omega_{i}}{\ell_{i}},
\end{array}
$$

and

$$
a_{1}^{\mathcal{T}, i}=-2 \ell_{i+2} \cos \left(\beta_{i+1}+\theta_{i}\right), \quad b_{1}^{\mathcal{T}, i}=-2 \ell_{i+2} \sin \left(\beta_{i+1}+\theta_{i}\right), \quad a_{2}^{\mathcal{T}, i}=-\frac{3 a_{1}^{\mathcal{T}, i}}{2 \ell_{i}}, \quad b_{2}^{\mathcal{T}, i}=-\frac{3 b_{1}^{\mathcal{T}, i}}{2 \ell_{i}} .
$$

The angles $\beta_{i}$ and $\theta_{i}$ are shown in Figure 1, and $\omega_{i}$ and $\zeta_{i}$ are given by

$$
\omega_{i}=\frac{3 \sin \left(\beta_{i+1}-\beta_{i+2}+2 \theta_{i}\right)}{\sin \beta_{i}}, \quad \zeta_{i}=\frac{\cos \left(\beta_{i+1}-\beta_{i+2}+2 \theta_{i}\right)}{\sin \beta_{i}},
$$

and we assume that quantities associated with $i$ are identical to those associated with $i \bmod 3$, e.g. $\beta_{4}=\beta_{1}, \ell_{5}=\ell_{2}$, etc. The local shape functions $\boldsymbol{s}_{2,1}^{\mathcal{T}, \mathcal{E}_{i}}$ and $\boldsymbol{s}_{2,2}^{\mathcal{T}, \mathcal{E}_{i}}$ are obtained by interchanging the first and the second rows of $\boldsymbol{s}_{1,1}^{\mathcal{T}, \mathcal{E}_{i}}$ and $\boldsymbol{s}_{1,2}^{\mathcal{T}, \mathcal{E}_{i}}$, respectively. In Figure 2, we have plotted the non-zero row of $s_{I, J}^{\mathcal{T}, \mathcal{E}}$ for all edges of a triangle. Note that the non-zero row of the local shape 


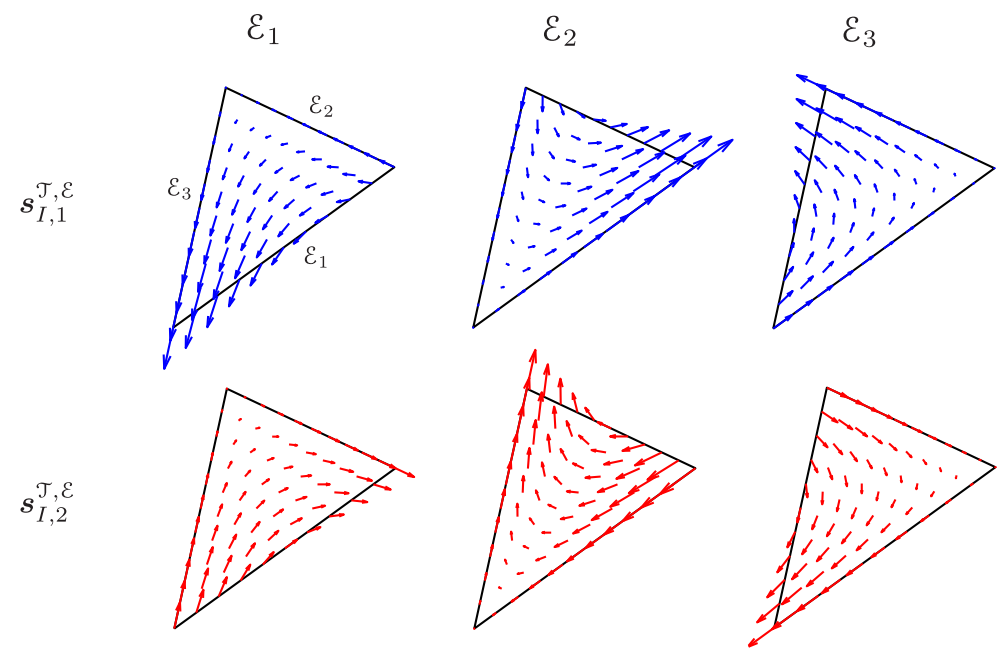

Figure 2: The nonzero row of $\boldsymbol{s}_{I, 1}^{\mathcal{T}, \mathcal{E}}$ (upper row) and $\boldsymbol{s}_{I, 2}^{\mathcal{T}, \mathcal{E}}$ (lower row) for all edges of a triangle.
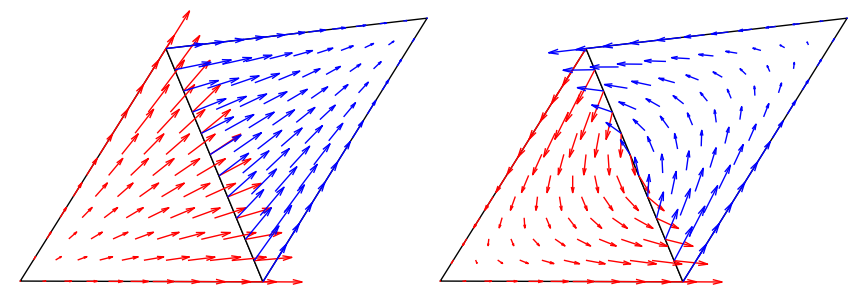

Figure 3: The nonzero rows of the global shape functions $\boldsymbol{S}_{I, 1}^{\mathcal{E}}$ (left) and $\boldsymbol{S}_{I, 2}^{\varepsilon}$ (right) associated to the common edge of two triangles. The components of fields normal to the common edge are single-valued.

functions associated to any edge is parallel to all the other edges. This simply follows from the duality relation (3.13) and is crucial for obtaining locally supported global shape functions.

For any $\boldsymbol{T} \in \mathcal{P}_{1}^{\mathbf{d}}\left(\mathcal{B}_{h}\right)$ and $\mathcal{E} \in \Delta_{1}\left(\mathcal{B}_{h}\right)$, the traction $\boldsymbol{T}\left(\mathbf{n}_{\mathcal{E}}\right)$ is a linear function of $s$ and $\left.\boldsymbol{T}\right|_{\mathcal{T}}$ is determined by the unique values of $\boldsymbol{T}\left(\mathbf{n}_{\mathcal{E}}\right), \forall \mathcal{E} \in \Delta_{1}(\mathcal{T})$. Therefore, one concludes that $\operatorname{dim} \mathcal{P}_{1}^{\mathbf{d}}\left(\mathcal{B}_{h}\right)=4 m_{\mathcal{E}}$, where $m_{\mathcal{E}}$ is the number of edges of $\mathcal{B}_{h}$. For obtaining global shape functions for $\mathcal{P}_{1}^{\mathbf{d}}\left(\mathcal{B}_{h}\right)$, we proceed as follows. Instead of the local shape functions $\boldsymbol{s}_{I, J}^{\mathcal{T}, \mathcal{E}}$, we consider the following local shape functions:

$$
\overline{\boldsymbol{s}}_{I, 1}^{\mathcal{T}, \mathcal{E}}:=\boldsymbol{s}_{I, 1}^{\mathcal{T}, \mathcal{E}}+\frac{\ell_{\mathcal{E}}}{2} \boldsymbol{s}_{I, 2}^{\mathcal{T}, \mathcal{E}}, \quad \text { and } \quad \bar{s}_{I, 2}^{\mathcal{T}, \mathcal{E}}:=-\frac{\ell_{\mathcal{E}}}{6} \boldsymbol{s}_{I, 2}^{\mathcal{T}, \mathcal{E}} .
$$

The basis dual to $\left\{\overline{\boldsymbol{s}}_{I, J}^{\mathcal{T}, \mathcal{E}}\right\}$ is denoted by $\left\{\bar{\psi}_{I, J}^{\mathcal{T}, \mathcal{E}}\right\}$, see (3.13). It is straightforward to show that

$$
\left\langle\left\langle\left.\left({\overrightarrow{\left(\bar{s}_{I, 1}^{\mathcal{T}, \mathcal{E}}\right.}}_{I}, \mathbf{n}_{\mathcal{E}}\right\rangle\right|_{\mathcal{E}}=\frac{1}{\ell_{\mathcal{E}}}, \quad\left\langle\left.\left\langle\left(\overrightarrow{\boldsymbol{s}}_{I, 2}^{\mathcal{T}, \mathcal{E}}\right)_{I}, \mathbf{n}_{\mathcal{E}}\right\rangle\right|_{\mathcal{E}}=-\frac{2}{\ell_{\varepsilon}^{2}} s+\frac{1}{\ell_{\varepsilon}} .\right.\right.\right.
$$

Next, we associate unit normal vectors $\hat{\mathbf{n}}_{\mathcal{E}}$ to all $\mathcal{E} \in \Delta_{1}\left(\mathcal{B}_{h}\right)$. Suppose that $\mathcal{E}$ is a common edge of $\mathcal{T}_{1}$ and $\mathcal{T}_{2}$ and let $\mathbf{n}_{\mathcal{E}}^{i}, i=1,2$, be the unit outward normal vector of $\mathcal{E}$ in $\mathcal{T}_{i}$. Then, one can define the global shape functions $\boldsymbol{S}_{I, J}^{\mathcal{E}}$ as $\left.\boldsymbol{S}_{I, J}^{\mathcal{E}}\right|_{\mathcal{T}}=\mathbf{0}$, if $\mathcal{T} \neq \mathcal{T}_{1}, \mathcal{T}_{2}$, and $\left.\boldsymbol{S}_{I, J}^{\mathcal{E}}\right|_{\mathcal{T}_{i}}=c_{J}^{i} \overline{\boldsymbol{s}}_{I, J}^{\mathcal{T}_{i}, \mathcal{E}}$, where $c_{1}^{i}=\left\langle\left\langle\hat{\mathbf{n}}_{\mathcal{E}}, \mathbf{n}_{\mathcal{E}}^{i}\right\rangle\right.$, and $c_{2}^{i}=1$. If $\mathcal{E}$ belongs only to a single triangle $\mathcal{T}$, then $\boldsymbol{S}_{I, J}^{\mathcal{E}}$ is nonzero only 
on $\mathcal{T}$ with $\left.\boldsymbol{S}_{I, J}^{\mathcal{E}}\right|_{\mathcal{T}}=\overline{\boldsymbol{s}}_{I, J}^{\mathcal{T}, \mathcal{E}}$. Figure 3 depicts the two global shape functions that are associated to the common edge of two triangles of a simple triangulation. Note that the nonzero rows of the global shape functions have single-valued normal components on the common edge. According to Theorem 6, this guarantees that the global shape functions are of $H^{\mathrm{d}}$-class.

We should also mention that global shape functions can be constructed with the local shape functions $\boldsymbol{s}_{I, J}^{\mathcal{T}, \mathcal{E}}$ as well. We have chosen the modified shape functions $\overline{\boldsymbol{s}}_{I, J}^{\mathcal{T}, \mathcal{E}}$ because they induce simple traction vectors on edges. For linear boundary traction vector fields, this makes enforcing the traction boundary conditions easier.

- $\mathcal{P}_{1}^{\mathbf{d}-}\left(\mathcal{B}_{h}\right)$ : Note that $\operatorname{dim} \mathcal{P}_{1}^{\ominus}\left(\otimes^{2} T \mathcal{T}\right)=6$, and one can write the consistent basis

$$
\Sigma^{\mathcal{T}, \mathbf{d}-}=\left\{\psi_{I}^{\mathcal{T}, \mathcal{E}}\right\}_{\substack{\mathcal{E} \in \Delta_{1}(\mathcal{T}) \\ I=1,2}}
$$

where $\psi_{I}^{\mathcal{T}, \mathcal{E}}=\psi_{I, 1}^{\mathcal{T}, \mathcal{E}}$, and $\psi_{I, 1}^{\mathcal{T}, \mathcal{E}}$ is defined in (3.11). Using the notation introduced in Figure 1, the local shape functions that constitute the dual basis of $\Sigma^{\mathcal{T}, \mathbf{d}-}$ can be written as

$$
\boldsymbol{s}_{1}^{\mathcal{T}, \mathcal{E}_{i}}(X, Y)=\frac{1}{2 A_{\mathcal{T}}}\left[\begin{array}{cc}
X-v_{i}^{x} & Y-v_{i}^{y} \\
0 & 0
\end{array}\right]
$$

and $\boldsymbol{s}_{2}^{\mathcal{T}, \mathcal{E}_{i}}$ is obtained by interchanging the rows of $\boldsymbol{s}_{1}^{\mathcal{T}, \mathcal{E}_{i}}$. It is straightforward to show that the normal traction of $\boldsymbol{s}_{1}^{\mathcal{T}, \mathcal{E}}$ on each edge is constant and satisfies $\boldsymbol{s}_{1}^{\mathcal{T}, \mathcal{E}}\left(\mathbf{n}_{\mathcal{E}}\right)=\frac{1}{\ell_{\mathcal{E}}}\left[\begin{array}{l}1 \\ 0\end{array}\right]$. In fact, it turns out that $\boldsymbol{s}_{I}^{\mathcal{T}, \mathcal{E}}=\overline{\boldsymbol{s}}_{I, 1}^{\mathcal{T}, \mathcal{E}}(\operatorname{see}(3.15))$. Consequently, $\operatorname{dim} \mathcal{P}_{1}^{\mathbf{d}-}\left(\mathcal{B}_{h}\right)=2 m_{\mathcal{E}}$, and the global shape functions associated to $\boldsymbol{s}_{I}^{\mathcal{T}, \mathcal{E}}$ are constructed similarly to $\boldsymbol{S}_{I, 1}^{\mathcal{E}}$ defined earlier for $\mathcal{P}_{1}^{\mathbf{d}}\left(\mathcal{B}_{h}\right)$.

- $\mathcal{P}_{2}^{\mathrm{d}}\left(\mathcal{B}_{h}\right)$ : Note that $\operatorname{dim} \mathcal{P}_{2}\left(\otimes^{2} T \mathcal{T}\right)=24$. One can write the consistent basis

$$
\Sigma^{\mathcal{T}, \mathbf{d}}=\left\{\psi_{I, J}^{\mathcal{T}, \mathcal{E}}, \psi_{I, J}^{\mathcal{T}, \mathcal{T}}\right\}_{\substack{\mathcal{E} \in \Delta_{1}(\mathcal{T}) \\ I=1,2, J=1,2,3}}
$$

where

$$
\psi_{I, J}^{\mathcal{T}, \mathcal{E}}(\boldsymbol{T})=\int_{\mathcal{E}} s^{J-1}\left\langle\left\langle\overrightarrow{\boldsymbol{T}}_{I}, \mathbf{n}_{\mathcal{E}}\right\rangle\right\rangle d s, \quad \psi_{I, J}^{\mathcal{T}, \mathcal{T}}(\boldsymbol{T})=\int_{\mathcal{T}}\left\langle\left\langle\overrightarrow{\boldsymbol{T}}_{I}, \boldsymbol{W}_{J}\right\rangle\right\rangle d A,
$$

with $\boldsymbol{W}_{1}=\left[\begin{array}{l}1 \\ 0\end{array}\right], \boldsymbol{W}_{2}=\left[\begin{array}{l}0 \\ 1\end{array}\right]$, and $\boldsymbol{W}_{3}=\left[\begin{array}{c}-Y \\ X\end{array}\right]$. In contrast to the first-order elements, it is not easy to explicitly write the associated dual local shape functions. In practice, it is easier to numerically calculate the local shape functions by using the duality relations similar to (3.13). Suppose that

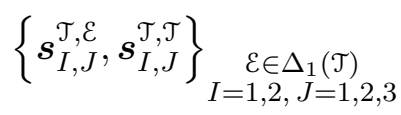

are the dual local shape functions and consider the following basis

$$
\left\{\overline{\boldsymbol{s}}_{I, J}^{\mathcal{T}, \mathcal{E}}, \boldsymbol{s}_{I, J}^{\mathcal{T}, \mathcal{T}}\right\} \underset{\substack{\mathcal{E} \in \Delta_{1}(\mathcal{T}) \\ I=1,2, J=1,2,3}}{ }
$$

where

$$
\bar{s}_{I, 1}^{\mathcal{T}, \mathcal{E}}:=s_{I, 1}^{\mathcal{T}, \mathcal{E}}+\frac{\ell_{\mathcal{E}}}{2} \boldsymbol{s}_{I, 2}^{\mathcal{T}, \mathcal{E}}+\frac{\ell_{\mathcal{E}}^{2}}{3} \boldsymbol{s}_{I, 3}^{\mathcal{T}, \mathcal{E}}, \quad \bar{s}_{I, 2}^{\mathcal{T}, \mathcal{E}}:=-\frac{\ell_{\mathcal{E}}}{6} \boldsymbol{s}_{I, 2}^{\mathcal{T}, \mathcal{E}}-\frac{\ell_{\mathcal{E}}^{2}}{6} \boldsymbol{s}_{I, 3}^{\mathcal{T}, \mathcal{E}},
$$




$$
\bar{s}_{I, 3}^{\mathcal{T}, \mathcal{E}}:=\frac{10}{3} s_{I, 1}^{\mathcal{T}, \mathcal{E}}+\frac{5 \ell_{\mathcal{E}}}{3} s_{I, 2}^{\mathcal{T}, \mathcal{E}}+\frac{17 \ell_{\mathcal{E}}^{2}}{15} \boldsymbol{s}_{I, 3}^{\mathcal{T}, \mathcal{E}}
$$

It is straightforward to show that

$$
\begin{aligned}
& \left.\left.\left\langle\overrightarrow{\left(\bar{s}_{I, 1}^{\mathcal{T}, \mathcal{E}}\right)_{I}}, \mathbf{n}_{\mathcal{E}}\right\rangle\right|_{\mathcal{E}}=\frac{1}{\ell_{\mathcal{E}}}, \quad \quad\left\langle\overrightarrow{\left(\overrightarrow{\boldsymbol{s}}_{I, 2}^{\mathcal{T}, \mathcal{E}}\right)_{I}}, \mathbf{n}_{\mathcal{E}}\right\rangle\right\rangle\left.\right|_{\mathcal{E}}=-\frac{2}{\ell_{\varepsilon}^{2}} s+\frac{1}{\ell_{\mathcal{E}}},
\end{aligned}
$$

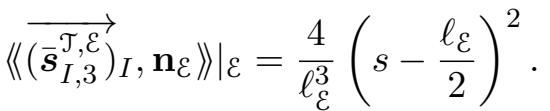

Consider the global shape functions $\boldsymbol{S}_{I, J}^{\mathcal{E}}, J=1,2$, introduced for $\mathcal{P}_{1}^{\mathbf{d}}\left(\mathcal{B}_{h}\right)$. The global shape functions associated to $\overline{\boldsymbol{s}}_{I, 1}^{\mathcal{T}, \mathcal{E}}$ and $\overline{\boldsymbol{s}}_{I, 3}^{\mathcal{T}, \mathcal{E}}$ are constructed similarly to $\boldsymbol{S}_{I, 1}^{\mathcal{E}}$, and those associated to $\overline{\boldsymbol{s}}_{I, 2}^{\mathcal{T}, \mathcal{E}}$ are obtained similarly to $\boldsymbol{S}_{I, 2}^{\mathcal{E}}$. Global shape functions $\boldsymbol{S}_{I, J}^{\mathcal{T}}$ associated to $\boldsymbol{s}_{I, J}^{\mathcal{T}, \mathcal{T}}$ are nonzero only on $\mathcal{T}$ with $\left.\boldsymbol{S}_{I, J}^{\mathcal{T}}\right|_{\mathcal{T}}=\boldsymbol{s}_{I, J}^{\mathcal{T}, \mathcal{T}}$.

- $\mathcal{P}_{2}^{\mathbf{d}-}\left(\mathcal{B}_{h}\right)$ : We have $\operatorname{dim} \mathcal{P}_{2}^{\ominus}\left(\otimes^{2} T \mathcal{T}\right)=16$, and one can write the following consistent basis

$$
\Sigma^{\mathcal{T}, \mathbf{d}-}=\left\{\psi_{I, J}^{\mathcal{T}, \mathcal{E}}, \psi_{I, J}^{\mathcal{T}, \mathcal{T}}\right\}_{\substack{\mathcal{E} \in \Delta_{1}(\mathcal{T}) \\ I, J=1,2}}
$$

where $\psi_{I, J}^{\mathcal{T}, \mathcal{E}}$ and $\psi_{I, J}^{\mathcal{T}, \mathcal{T}}$ are defined in (3.16). In practice, it is easier to numerically calculate the associated dual local shape functions

$$
\left\{s_{I, J}^{\mathcal{T}, \mathcal{E}}, s_{I, J}^{\mathcal{T}, \mathcal{T}}\right\}_{\substack{\mathcal{E} \in \Delta_{1}(\mathcal{T}) \\ I, J=1,2}}
$$

For calculating the global shape functions, one can use the basis

$$
\left\{\bar{s}_{I, J, \mathcal{T}, \mathcal{E}}^{\mathcal{T}, \mathcal{T}, \mathcal{T}}\right\}_{\substack{\mathcal{E} \in \Delta_{1}(\mathcal{T}) \\ I, J=1,2}}
$$

where

$$
\overline{\boldsymbol{s}}_{I, 1}^{\mathcal{T}, \mathcal{E}}:=\boldsymbol{s}_{I, 1}^{\mathcal{T}, \mathcal{E}}+\frac{\ell_{\mathcal{E}}}{2} \boldsymbol{s}_{I, 2}^{\mathcal{T}, \mathcal{E}}, \quad \overline{\boldsymbol{s}}_{I, 2}^{\mathcal{T}, \mathcal{E}}:=-\frac{\ell_{\mathcal{E}}}{6} \boldsymbol{s}_{I, 2}^{\mathcal{T}, \mathcal{E}}
$$

One can show that

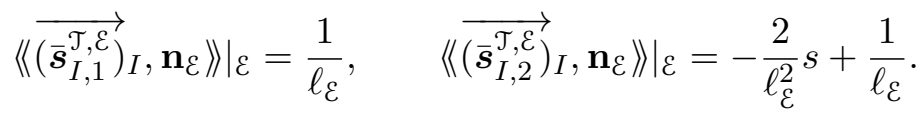

The global shape functions associated to $\overline{\boldsymbol{s}}_{I, 1}^{\mathcal{T}, \mathcal{E}}\left(\overline{\boldsymbol{s}}_{I, 2}^{\mathcal{T}, \mathcal{E}}\right)$ are constructed similarly to the global shape functions $\boldsymbol{S}_{I, 1}^{\mathcal{E}}\left(\boldsymbol{S}_{I, 2}^{\mathcal{E}}\right)$ introduced for $\mathcal{P}_{1}^{\mathrm{d}}\left(\mathcal{B}_{h}\right)$. Global shape functions $\boldsymbol{S}_{I, J}^{\mathcal{T}}$ associated to $\boldsymbol{s}_{I, J}^{\mathcal{T}, \mathcal{T}}$ are nonzero only on $\mathcal{T}$ with $\left.\boldsymbol{S}_{I, J}^{\mathcal{T}}\right|_{\mathcal{T}}=\boldsymbol{s}_{I, J}^{\mathcal{T}, \mathcal{T}}$.

Example 10. In this example, we calculate the local and global shape functions for the $H^{\mathbf{c}}$-conformal finite element spaces $\mathcal{P}_{r}^{\mathbf{c}}\left(\mathcal{B}_{h}\right)$ and $\mathcal{P}_{r}^{\mathbf{c}-}\left(\mathcal{B}_{h}\right)$, with $r=1,2$.

- $\mathcal{P}_{1}^{\mathbf{c}}\left(\mathcal{B}_{h}\right)$ : One can write the consistent basis

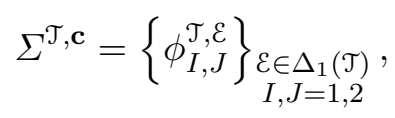




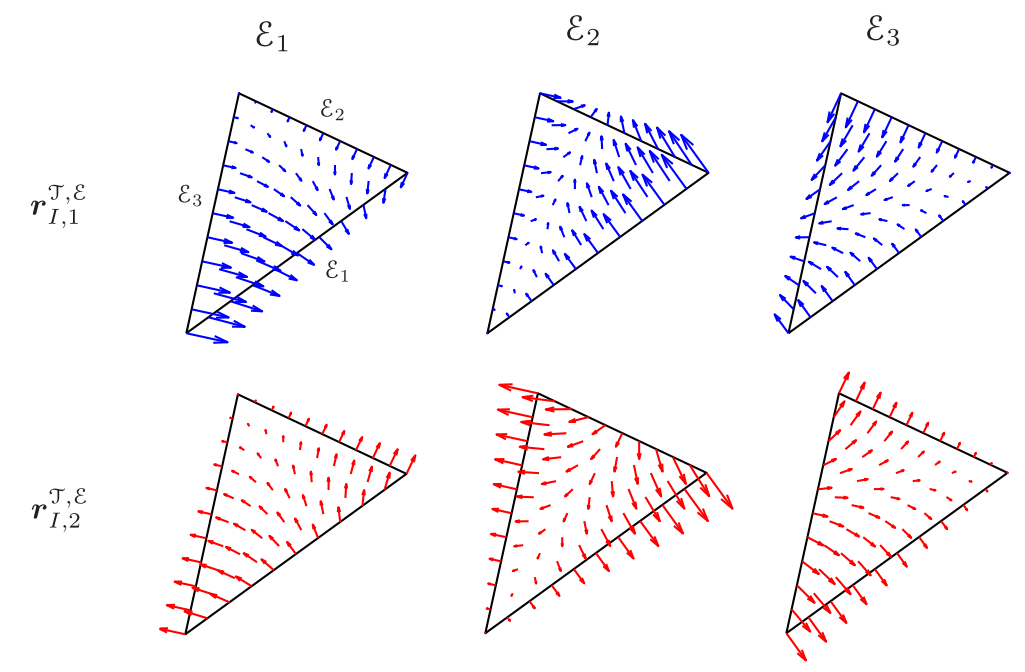

Figure 4: The nonzero row of $\boldsymbol{r}_{I, 1}^{\mathcal{T}, \mathcal{E}}$ (upper row) and $\boldsymbol{r}_{I, 2}^{\mathcal{T}, \mathcal{E}}$ (lower row) for all edges of a triangle.

where

$$
\phi_{I, J}^{\mathcal{T}, \mathcal{E}}(\boldsymbol{T})=\int_{\mathcal{E}} s^{J-1}\left\langle\left\langle\overrightarrow{\boldsymbol{T}}_{I}, \mathbf{t}_{\mathcal{E}}\right\rangle\right\rangle d s
$$

Using the notation of Figure 1, the dual local shape functions $\boldsymbol{r}_{I, J}^{\mathcal{T}, \mathcal{E}}$ can be written as

$\boldsymbol{r}_{1, J}^{\mathcal{T}, \mathcal{E}_{i}}(X, Y)=$
$\frac{1}{A_{\mathcal{T}}}\left[\begin{array}{cc}-b_{X, J}^{\mathcal{T}, i} \cdot\left(X-v_{i+1}^{x}\right)-b_{Y, J}^{\mathcal{T}, i} \cdot\left(Y-v_{i+1}^{y}\right)-b_{J}^{\mathcal{T}, i} & a_{X, J}^{\mathcal{T}, i} \cdot\left(X-v_{i+1}^{x}\right)+a_{Y, J}^{\mathcal{T}, i} \cdot\left(Y-v_{i+1}^{y}\right)+a_{J}^{\mathcal{T}, i} \\ 0 & 0\end{array}\right]$,

where the coefficients are defined in (3.14). The local shape functions $\boldsymbol{r}_{2, J}^{\mathcal{T}, \mathcal{E}_{i}}$ are obtained by interchanging the rows of $\boldsymbol{r}_{1, J}^{\mathcal{T}, \mathcal{E}_{i}}$. Note that for any $I$ and $J$, the non-zero row of $\boldsymbol{s}_{I, J}^{\mathcal{T}, \mathcal{E}_{i}}$ introduced in (3.12) is normal to that of $\boldsymbol{r}_{I, J}^{\mathcal{T}, \mathcal{E}_{i}}$. Figure 4 depicts the non-zero row of $\boldsymbol{r}_{I, J}^{\mathcal{T}, \mathcal{E}}$ for all edges of a triangle. The non-zero row of the local shape functions associated to any edge is normal to all the other edges.

Since any $\boldsymbol{T} \in \mathcal{P}_{1}^{\mathbf{c}}\left(\mathcal{B}_{h}\right)$ is determined by its linear tractions $\boldsymbol{T}\left(\mathbf{t}_{\varepsilon}\right)$ along edges, one concludes that $\operatorname{dim} \mathcal{P}_{1}^{\mathbf{c}}\left(\mathcal{B}_{h}\right)=4 m_{\varepsilon}$. For writing global shape functions, similar to $\mathcal{P}_{1}^{\mathbf{d}}\left(\mathcal{B}_{h}\right)$, we consider the following local shape functions:

$$
\overline{\boldsymbol{r}}_{I, 1}^{\mathcal{T}, \mathcal{E}}:=\boldsymbol{r}_{I, 1}^{\mathcal{T}, \mathcal{E}}+\frac{\ell_{\mathcal{\varepsilon}}}{2} \boldsymbol{r}_{I, 2}^{\mathcal{T}, \mathcal{E}}, \quad \text { and } \quad \overline{\boldsymbol{r}}_{I, 2}^{\mathcal{T}, \mathcal{E}}:=-\frac{\ell_{\mathcal{\varepsilon}}}{6} \boldsymbol{r}_{I, 2}^{\mathcal{T}, \mathcal{E}},
$$

that satisfy

$$
\left\langle\left.\left\langle\overrightarrow{\left(\overline{\boldsymbol{r}}_{I, 1}^{\mathcal{T}, \varepsilon}\right)_{I}}, \mathbf{t}_{\mathcal{\varepsilon}}\right\rangle\right|_{\mathcal{E}}=\frac{1}{\ell_{\varepsilon}}, \quad\left\langle\left.\left\langle\left(\overrightarrow{\left(\overline{\boldsymbol{r}}_{I, 2}^{\mathcal{T}, \varepsilon}\right.}\right)_{I}, \mathbf{t}_{\mathcal{\varepsilon}}\right\rangle\right|_{\mathcal{E}}=-\frac{2}{\ell_{\varepsilon}^{2}} s+\frac{1}{\ell_{\varepsilon}} .\right.\right.
$$

The basis dual to $\left\{\overline{\boldsymbol{r}}_{I, J}^{\mathcal{T}, \mathcal{E}}\right\}$ is denoted by $\left\{\bar{\phi}_{I, J}^{\mathcal{T}, \mathcal{E}}\right\}$. To obtain the global shape functions $\boldsymbol{R}_{I, J}^{\mathcal{E}}$, one associates unit tangent vectors $\hat{\mathbf{t}}_{\varepsilon}$ to all $\mathcal{E} \in \Delta_{1}\left(\mathcal{B}_{h}\right)$. Suppose that $\mathcal{E}$ is a common edge of $\mathcal{T}_{1}$ and $\mathcal{T}_{2}$ and suppose that $\mathbf{t}_{\mathcal{E}}^{i}, i=1,2$, is the oriented unit tangent vector of $\mathcal{E}$ in $\mathcal{T}_{i}$. Then, the nonzero restrictions of the global shape functions $\boldsymbol{R}_{I, J}^{\mathcal{E}}$ are given by $\boldsymbol{R}_{I, J}^{\mathcal{E}} \mid \mathcal{T}_{i}=e_{J}^{i} \overline{\boldsymbol{r}}_{I, J}^{\mathcal{T}_{i}, \mathcal{E}}$, 


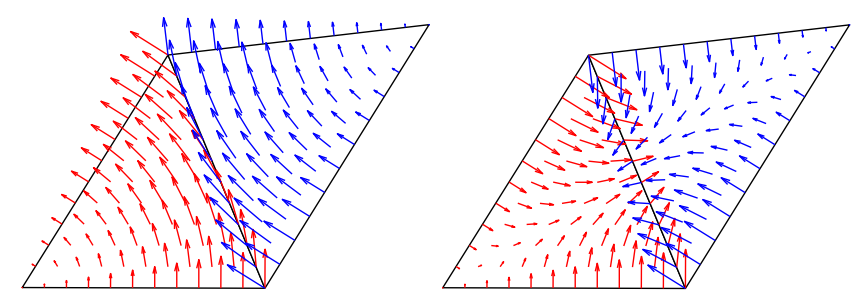

Figure 5: The nonzero rows of the global shape functions $\boldsymbol{R}_{I, 1}^{\mathcal{E}}$ (left) and $\boldsymbol{R}_{I, 2}^{\mathcal{E}}$ (right) associated to the common edge of two triangles. The components of fields tangent to the common edge are single-valued.

where $e_{1}^{i}=\left\langle\left\langle\hat{\mathbf{t}}_{\mathcal{E}}, \mathbf{t}_{\mathcal{E}}^{i}\right\rangle\right\rangle$, and $e_{2}^{i}=1$. If $\mathcal{E}$ belongs to a single triangle $\mathcal{T}$, then the only nonzero restriction is given by $\left.\boldsymbol{R}_{I, J}^{\mathcal{E}}\right|_{\mathcal{T}}=\overline{\boldsymbol{r}}_{I, J}^{\mathcal{T}, \mathcal{E}}$. In Figure 5, we have plotted the nonzero rows of the global shape functions $\boldsymbol{R}_{I, J}^{\mathcal{E}}$ associated to the common edge of two triangles. Note that the tangent components of vector fields along edges are single-valued.

- $\mathcal{P}_{1}^{\mathbf{c}-}\left(\mathcal{B}_{h}\right)$ : Note that $\operatorname{dim} \mathcal{P}_{1}^{-}\left(\otimes^{2} T \mathcal{T}\right)=6$, and one obtains the consistent basis

$$
\Sigma^{\mathcal{T}, \mathbf{c}-}=\left\{\phi_{I}^{\mathcal{T}, \mathcal{E}}\right\}_{\substack{\mathcal{E} \in \Delta_{1}(\mathcal{T}) \\ I=1,2}}
$$

where $\phi_{I}^{\mathcal{T}, \mathcal{E}}=\phi_{I, 1}^{\mathcal{T}, \mathcal{E}}($ see $(3.17))$. By using the notation of Figure 1, one can write the dual local shape functions as

$$
\boldsymbol{r}_{1}^{\mathcal{T}, \mathcal{E}_{i}}(X, Y)=\frac{1}{2 A_{\mathcal{T}}}\left[\begin{array}{cc}
-\left(Y-v_{i}^{y}\right) & X-v_{i}^{x} \\
0 & 0
\end{array}\right]
$$

and $\boldsymbol{r}_{2}^{\mathcal{T}, \mathcal{E}_{i}}$ is obtained by interchanging the rows of $\boldsymbol{r}_{1}^{\mathcal{T}, \mathcal{E}_{i}}$. One can show that $\boldsymbol{r}_{1}^{\mathcal{T}, \mathcal{E}}\left(\mathbf{t}_{\varepsilon}\right)=\frac{1}{\ell_{\varepsilon}}\left[\begin{array}{l}1 \\ 0\end{array}\right]$, and it turns out that $\boldsymbol{r}_{I}^{\mathcal{T}, \mathcal{E}}=\overline{\boldsymbol{r}}_{I, 1}^{\mathcal{T}, \mathcal{E}}($ see $(3.18))$. Hence, $\operatorname{dim} \mathcal{P}_{1}^{\mathbf{c}-}\left(\mathcal{B}_{h}\right)=2 m_{\mathcal{E}}$, and the global shape functions $\boldsymbol{R}_{I, 1}^{\mathcal{E}}$ introduced for $\mathcal{P}_{1}^{\mathbf{c}}\left(\mathcal{B}_{h}\right)$ are locally supported global shape functions for $\mathcal{P}_{1}^{\mathbf{c}-}\left(\mathcal{B}_{h}\right)$.

- $\mathcal{P}_{2}^{\mathbf{c}}\left(\mathcal{B}_{h}\right)$ : We can write the following consistent basis

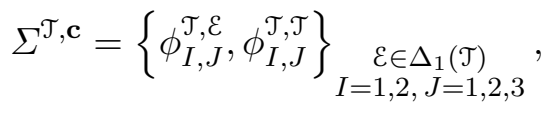

where

$$
\phi_{I, J}^{\mathcal{T}, \mathcal{E}}(\boldsymbol{T})=\int_{\mathcal{E}} s^{J-1}\left\langle\left\langle\overrightarrow{\boldsymbol{T}}_{I}, \mathbf{t}_{\mathcal{E}}\right\rangle d s, \quad \phi_{I, J}^{\mathcal{T}, \mathcal{T}}(\boldsymbol{T})=\int_{\mathcal{T}}\left\langle\left\langle\overrightarrow{\boldsymbol{T}}_{I}, \boldsymbol{W}_{J}\right\rangle d A,\right.\right.
$$

with $\boldsymbol{W}_{1}=\left[\begin{array}{l}1 \\ 0\end{array}\right], \boldsymbol{W}_{2}=\left[\begin{array}{l}0 \\ 1\end{array}\right]$, and $\boldsymbol{W}_{3}=\left[\begin{array}{c}X \\ Y\end{array}\right]$. It is not straightforward to write the explicit form of the associated dual shape functions. In practice, one can use the duality relations similar to (3.13) to numerically calculate the shape functions. Let

$$
\left\{\boldsymbol{r}_{I, J}^{\mathcal{T}, \mathcal{E}}, \boldsymbol{r}_{I, J}^{\mathcal{T}, \mathcal{T}}\right\} \underset{\substack{\mathcal{E} \in \Delta_{1}(\mathcal{T}) \\ I=1,2, J=1,2,3}}{ }
$$

be the dual local shape functions. To obtain the global shape functions, we consider the following basis

$$
\left\{\overline{\boldsymbol{r}}_{I, J}^{\mathcal{T}, \mathcal{E}}, \boldsymbol{r}_{I, J}^{\mathcal{T}, \mathcal{T}}\right\} \underset{\substack{\mathcal{E} \in \Delta_{1}(\mathcal{T}) \\ I=1,2, J=1,2,3}}{ }
$$


where

$$
\begin{aligned}
& \overline{\boldsymbol{r}}_{I, 1}^{\mathcal{T}, \mathcal{E}}:=\boldsymbol{r}_{I, 1}^{\mathcal{T}, \mathcal{E}}+\frac{\ell_{\mathcal{E}}}{2} \boldsymbol{r}_{I, 2}^{\mathcal{T}, \mathcal{E}}+\frac{\ell_{\mathcal{E}}^{2}}{3} \boldsymbol{r}_{I, 3}^{\mathcal{T}, \mathcal{E}}, \quad \overline{\boldsymbol{r}}_{I, 2}^{\mathcal{T}, \mathcal{E}}:=-\frac{\ell_{\mathcal{E}}}{6} \boldsymbol{r}_{I, 2}^{\mathcal{T}, \mathcal{E}}-\frac{\ell_{\mathcal{E}}^{2}}{6} \boldsymbol{r}_{I, 3}^{\mathcal{T}, \mathcal{E}}, \\
& \overline{\boldsymbol{r}}_{I, 3}^{\mathcal{T}, \mathcal{E}}:=\frac{10}{3} \boldsymbol{r}_{I, 1}^{\mathcal{T}, \mathcal{E}}+\frac{5 \ell_{\mathcal{E}}}{3} \boldsymbol{r}_{I, 2}^{\mathcal{T}, \mathcal{E}}+\frac{17 \ell_{\mathcal{E}}^{2}}{15} \boldsymbol{r}_{I, 3}^{\mathcal{T}, \mathcal{E}} .
\end{aligned}
$$

One can show that

$$
\begin{aligned}
& \left\langle\left.\left\langle\left(\overline{\boldsymbol{r}}_{I, 1}^{\mathcal{T}, \mathcal{E}}\right)_{I}, \mathbf{t}_{\mathcal{E}}\right\rangle\right|_{\mathcal{E}}=\frac{1}{\ell_{\varepsilon}},\right. \\
& \left.\left\langle\left(\overrightarrow{\boldsymbol{r}}_{I, 2}^{\mathcal{T}, \mathcal{E}}\right)_{I}, \mathbf{t}_{\mathcal{E}}\right\rangle\right|_{\mathcal{E}}=-\frac{2}{\ell_{\varepsilon}^{2}} s+\frac{1}{\ell_{\varepsilon}}, \\
& \left\langle\left.\left\langle\left(\overrightarrow{\boldsymbol{r}}_{I, 3}^{\mathcal{T}, \mathcal{E}}\right)_{I}, \mathbf{t}_{\mathcal{E}}\right\rangle\right|_{\mathcal{E}}=\frac{4}{\ell_{\mathcal{E}}^{3}}\left(s-\frac{\ell_{\mathcal{E}}}{2}\right)^{2} .\right.
\end{aligned}
$$

The global shape functions can be calculated by using the basis (3.20) as follows. Consider the global shape functions $\boldsymbol{R}_{I, J}^{\mathcal{E}}, J=1,2$, introduced for $\mathcal{P}_{1}^{\mathbf{c}}\left(\mathcal{B}_{h}\right)$. The global shape functions associated to $\overline{\boldsymbol{r}}_{I, 1}^{\mathcal{T}, \mathcal{E}}$ and $\overline{\boldsymbol{r}}_{I, 3}^{\mathcal{T}, \mathcal{E}}$ are constructed similarly to $\boldsymbol{R}_{I, 1}^{\mathcal{E}}$, and those associated to $\overline{\boldsymbol{r}}_{I, 2}^{\mathcal{T}, \mathcal{E}}$ are obtained similarly to $\boldsymbol{R}_{I, 2}^{\mathcal{E}}$. The global shape functions $\boldsymbol{R}_{I, J}^{\mathcal{T}}$ associated to $\boldsymbol{r}_{I, J}^{\mathcal{T}, \mathcal{T}}$ are nonzero only on $\mathcal{T}$ with $\left.\boldsymbol{R}_{I, J}^{\mathcal{T}}\right|_{\mathcal{T}}=\boldsymbol{r}_{I, J}^{\mathcal{T}, \mathcal{T}}$.

- $\mathcal{P}_{2}^{\mathbf{c}-}\left(\mathcal{B}_{h}\right)$ : Note that $\operatorname{dim} \mathcal{P}_{2}^{-}\left(\otimes^{2} T \mathcal{T}\right)=16$, and one can write the consistent basis

$$
\Sigma^{\mathcal{T}, \mathbf{c}-}=\left\{\phi_{I, J}^{\mathcal{T}, \mathcal{E}}, \phi_{I, J}^{\mathcal{T}, \mathcal{T}}\right\}_{\substack{\mathcal{E} \in \Delta_{1}(\mathcal{T}) \\ I, J=1,2}},
$$

where $\phi_{I, J}^{\mathcal{T}, \mathcal{E}}$ and $\phi_{I, J}^{\mathcal{T}, \mathcal{T}}$ are defined in (3.19). It is easier to numerically calculate the associated dual local shape functions

$$
\left\{\boldsymbol{r}_{I, J}^{\mathcal{T}, \mathcal{E}}, \boldsymbol{r}_{I, J}^{\mathcal{T}, \mathcal{T}}\right\}_{\substack{\mathcal{E} \in \Delta_{1}(\mathcal{T}) \\ I, J=1,2}}
$$

For calculating the global shape functions, one can use the following basis

$$
\left\{\overline{\boldsymbol{r}}_{I, J}^{\mathcal{T}, \mathcal{E}}, \boldsymbol{r}_{I, J}^{\mathcal{T}, \mathcal{T}}\right\}_{\substack{\mathcal{E} \in \Delta_{1}(\mathcal{T}) \\ I, J=1,2}}
$$

with

$$
\overline{\boldsymbol{r}}_{I, 1}^{\mathcal{T}, \mathcal{E}}:=\boldsymbol{r}_{I, 1}^{\mathcal{T}, \mathcal{E}}+\frac{\ell_{\mathcal{\varepsilon}}}{2} \boldsymbol{r}_{I, 2}^{\mathcal{T}, \mathcal{E}}, \quad \overline{\boldsymbol{r}}_{I, 2}^{\mathcal{T}, \mathcal{E}}:=-\frac{\ell_{\mathcal{\varepsilon}}}{6} \boldsymbol{r}_{I, 2}^{\mathcal{T}, \mathcal{E}}
$$

It is straightforward to show that

$$
\left\langle\left.\left\langle\overrightarrow{\left(\overline{\boldsymbol{r}}_{I, 1}^{\mathcal{T}, \mathcal{E}}\right)_{I}}, \mathbf{t}_{\mathcal{E}}\right\rangle\right|_{\mathcal{E}}=\frac{1}{\ell_{\varepsilon}},\left.\quad\left\langle\overrightarrow{\left(\overline{\boldsymbol{r}}_{I, 2}^{\mathcal{T}, \mathcal{E}}\right)_{I}}, \mathbf{t}_{\mathcal{\varepsilon}}\right\rangle\right|_{\mathcal{E}}=-\frac{2}{\ell_{\varepsilon}^{2}} s+\frac{1}{\ell_{\varepsilon}} .\right.
$$

The global shape functions associated to $\overline{\boldsymbol{r}}_{I, 1}^{\mathcal{T}, \mathcal{E}}\left(\overline{\boldsymbol{r}}_{I, 2}^{\mathcal{T}, \mathcal{E}}\right)$ are constructed similarly to the global shape functions $\boldsymbol{R}_{I, 1}^{\mathcal{E}}\left(\boldsymbol{R}_{I, 2}^{\mathcal{E}}\right)$ introduced for $\mathcal{P}_{1}^{\mathbf{c}}\left(\mathcal{B}_{h}\right)$. Global shape functions $\boldsymbol{R}_{I, J}^{\mathcal{T}}$ associated to $\boldsymbol{r}_{I, J}^{\mathcal{T}, \mathcal{T}}$ are nonzero only on $\mathcal{T}$ with $\left.\boldsymbol{R}_{I, J}^{\mathcal{T}}\right|_{\mathcal{T}}=\boldsymbol{r}_{I, J}^{\mathcal{T}, \mathcal{T}}$.

Remark 11. Local degrees of freedom derived in the previous examples are similar to those of some classical edge and face finite elements for vector fields. This means that the above finite elements are the tensorial analogues of some vectorial finite elements, which are obtained by identifying a secondorder tensor field with two vector fields associated to its rows. Table 2 summarizes the correspondence 
Table 2: Tensorial analogues of some classical finite elements for vector fields.

\begin{tabular}{ll}
\hline \multicolumn{1}{c}{ Vector Fields } & \multicolumn{1}{c}{ Second-Order Tensors } \\
\hline Nédélec $2^{\text {nd }-k i n d ~} H($ curl $)$ element $[35]$ & $\left(\mathcal{T}, \mathcal{P}_{r}\left(\otimes^{2} T \mathcal{T}\right), \Sigma^{\mathcal{T}, \mathbf{c}}\right)$ \\
Nédélec $1^{\text {st }-k i n d ~} H($ curl $)$ element $[35]$ & $\left(\mathcal{T}, \mathcal{P}_{r}^{-}\left(\otimes^{2} T \mathcal{T}\right), \Sigma^{\mathcal{T}, \mathbf{c}-}\right)$ \\
Brezzi-Douglas-Marini $H($ div $)$ element $[34]$ & $\left(\mathcal{T}, \mathcal{P}_{r}\left(\otimes^{2} T \mathcal{T}\right), \Sigma^{\mathcal{T}, \mathbf{d}}\right)$ \\
Raviart-Thomas $H($ div $)$ element $[33]$ & $\left(\mathcal{T}, \mathcal{P}_{r}^{\ominus}\left(\otimes^{2} T \mathcal{T}\right), \Sigma^{\mathcal{T}, \mathbf{d}-}\right)$ \\
\hline
\end{tabular}

between the finite elements (3.10) and some classical vectorial finite elements. The procedure for determining degrees of freedom discussed in this section follows from the finite element exterior calculus introduced in $[29,30]$. This approach provides a systematic way for writing finite elements of an arbitrary order.

\subsubsection{Discrete Hilbert Complexes}

Using the finite element spaces introduced in the previous section, one can discretize the Hilbert complexes (2.1) and (2.2). More specifically, one can discretize (2.1) as follows:

$$
\begin{aligned}
& 0 \longrightarrow \mathcal{P}_{r+2}^{1}\left(T \mathcal{B}_{h}, \Gamma_{d}\right) \stackrel{\text { grad }}{\longrightarrow} \mathcal{P}_{r+1}^{\mathbf{c}}\left(\mathcal{B}_{h}, \Gamma_{d}\right) \stackrel{\text { c }}{\longrightarrow} \mathcal{P}_{r}\left(T \mathcal{B}_{h}\right) \longrightarrow 0, \\
& 0 \longrightarrow \mathcal{P}_{r+1}^{1}\left(T \mathcal{B}_{h}, \Gamma_{d}\right) \stackrel{\text { grad }}{\longrightarrow} \mathcal{P}_{r+1}^{\mathbf{c}-}\left(\mathcal{B}_{h}, \Gamma_{d}\right) \stackrel{\text { c }}{\longrightarrow} \mathcal{P}_{r}\left(T \mathcal{B}_{h}\right) \longrightarrow 0 .
\end{aligned}
$$

Analogously, one can also write the following discrete complexes for (2.2):

$$
\begin{aligned}
& 0 \longrightarrow \mathcal{P}_{r+2}^{1}\left(T \mathcal{B}_{h}, \Gamma_{t}\right) \stackrel{\text { s }}{\longrightarrow} \mathcal{P}_{r+1}^{\mathbf{d}}\left(\mathcal{B}_{h}, \Gamma_{t}\right) \stackrel{- \text { div }}{\longrightarrow} \mathcal{P}_{r}\left(T \mathcal{B}_{h}\right) \longrightarrow 0, \\
& 0 \longrightarrow \mathcal{P}_{r+1}^{1}\left(T \mathcal{B}_{h}, \Gamma_{t}\right) \stackrel{\text { s }}{\longrightarrow} \mathcal{P}_{r+1}^{\mathbf{d}-}\left(\mathcal{B}_{h}, \Gamma_{t}\right) \stackrel{- \text { div }}{\longrightarrow} \mathcal{P}_{r}\left(T \mathcal{B}_{h}\right) \longrightarrow 0 .
\end{aligned}
$$

The above complexes are the analogues of the discrete complexes (3.1) and (3.5) for the triangulation $\mathcal{B}_{h}$. By using the bounded cochain projections between differential forms and piecewise polynomial differential forms discussed in [29, 42], one can obtain bounded cochain projections between the Hilbert complexes (2.1) and (2.2) and the above discrete complexes. As an implication of these projections, one can apply the general approximation theory of Hilbert complexes introduced by Arnold et al. [30] to approximate (2.1) and (2.2) by means of the above discrete complexes. In particular, one can derive stable mixed finite element methods for approximating the tensor Laplacian

$$
\mathscr{L}(T):=\mathrm{s} \circ \mathrm{c}(\boldsymbol{T})-\operatorname{grad} \circ \operatorname{div}(T)
$$

subject to suitable boundary conditions. Moreover, one can show that regardless of the refinement level of the mesh $\mathcal{B}_{h}$, the discrete complexes (3.21) and (3.22) inherit all the information regarding the topological properties of $\mathcal{B}_{h}, \Gamma_{d}$, and $\Gamma_{t}$, which is contained in (2.1) and (2.2). ${ }^{3}$

\subsection{Compatible-Strain Mixed Finite Element Methods}

We are now in a position to introduce suitable mixed finite element methods for approximating the 2D nonlinear elasticity problem (2.8) by means of finite element spaces introduced earlier. More

\footnotetext{
${ }^{3}$ Strictly speaking, the above cochain projections induce isomorphisms between cohomology groups of (2.1) and (2.2) and those of their discrete counterparts.
} 


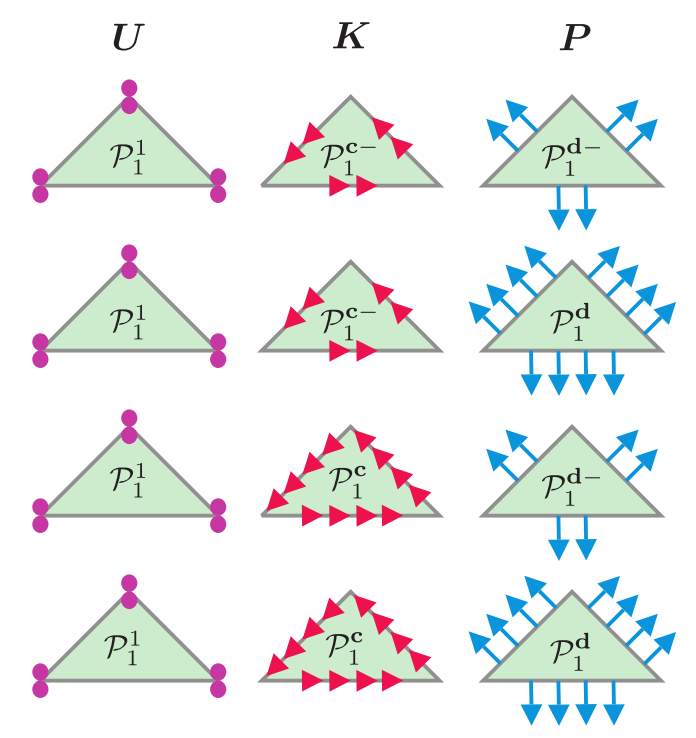

Figure 6: The conventional finite element diagrams for all the first-order finite elements for $(\boldsymbol{U}, \boldsymbol{K}, \boldsymbol{P})$. Each row gives a first-order finite element method for approximating (2.8). In the numerical examples that are considered in this work, the combination of the third row has the best performance among all the first-order combinations. Note that each arrow on (normal to) an edge represents a degree of freedom of the corresponding finite element associated to that edge, see Examples 9 and 10 .

specifically, suppose $p, q, r>0$ are integers, let $V_{p}^{\mathbf{c}}$ be either $\mathcal{P}_{p}^{\mathbf{c}}\left(\mathcal{B}_{h}\right)$ or $\mathcal{P}_{p}^{\mathbf{c}-}\left(\mathcal{B}_{h}\right)$, and let $V_{q}^{\mathbf{d}}$ be either $\mathcal{P}_{q}^{\mathrm{d}}\left(\mathcal{B}_{h}\right)$ or $\mathcal{P}_{q}^{\mathrm{d}-}\left(\mathcal{B}_{h}\right)$. Also let $V_{q}^{\mathrm{d}}\left(\Gamma_{t}\right):=V_{q}^{\mathrm{d}} \cap H^{\mathrm{d}}\left(\mathcal{B}, \Gamma_{t}\right)$. We write the following mixed finite element methods for $(2.8)$.

Given a body force $\boldsymbol{B}$ of $L^{2}$-class, a boundary displacement $\overline{\boldsymbol{U}}$ on $\Gamma_{d}$ of $H^{1 / 2}$-class, and a boundary traction $\overline{\boldsymbol{T}}$ on $\Gamma_{t}$ of $L^{2}$-class, find $\left(\boldsymbol{U}_{h}, \boldsymbol{K}_{h}, \boldsymbol{P}_{h}\right) \in \mathcal{P}_{r}^{1}\left(T \mathcal{B}_{h}\right) \times V_{p}^{\mathbf{c}} \times V_{q}^{\mathbf{d}}$ such that

$$
\begin{aligned}
\left\langle\left\langle\boldsymbol{P}_{h}, \operatorname{grad} \boldsymbol{\Upsilon}\right\rangle\right\rangle_{L^{2}} & =\left\langle\left\langle\rho_{0} \boldsymbol{B}, \boldsymbol{\Upsilon}\right\rangle\right\rangle_{L^{2}}+\int_{\Gamma_{t}}\langle\overline{\boldsymbol{T}}, \boldsymbol{\Upsilon}\rangle d s, & & \forall \boldsymbol{\Upsilon} \in \mathcal{P}_{r}^{1}\left(T \mathcal{B}_{h}, \Gamma_{d}\right), \\
\left\langle\left\langle\widetilde{\boldsymbol{P}}\left(\boldsymbol{K}_{h}\right), \boldsymbol{\kappa}\right\rangle\right\rangle_{L^{2}}-\left\langle\left\langle\boldsymbol{P}_{h}, \boldsymbol{\kappa}\right\rangle\right\rangle_{L^{2}} & =\mathbf{0}, & & \forall \boldsymbol{\kappa} \in V_{p}^{\mathbf{c}}, \\
\left\langle\left\langle\boldsymbol{U}_{h}, \operatorname{div} \boldsymbol{\pi}\right\rangle\right\rangle_{L^{2}}+\left\langle\left\langle\boldsymbol{K}_{h}, \boldsymbol{\pi}\right\rangle\right\rangle_{L^{2}} & \left.=\int_{\Gamma_{d}}\langle\overline{\boldsymbol{U}}, \boldsymbol{\pi}(\boldsymbol{N})\rangle\right\rangle d s, & & \forall \boldsymbol{\pi} \in V_{q}^{\mathbf{d}}\left(\Gamma_{t}\right) .
\end{aligned}
$$

The choice of $H^{\mathbf{c}}$-conformal spaces for displacement gradients follows from the Hilbert complex (2.1). This choice can also be considered as a consequence of the weak compatibility equations for $L^{2}$ displacement gradients which are implicitly given by (2.1) (also see the discussions in [40]). Therefore, we call finite element methods introduced in (3.24) the compatible-strain mixed finite element methods (CSFEM).

It is well-known that consistent discretizations of a well-posed mixed formulation may not necessarily lead to stable mixed finite element methods as solution and test spaces need to satisfy certain compatibility conditions, e.g. see the Ladyzhenskaya-Babuška-Brezzi condition [23, 24, 25]. The general discussions in $[30, \S 3]$ regarding approximations of the abstract Hodge Laplacian suggest that one can obtain stable mixed finite element methods for the tensor Laplacian (3.23) by using the discrete Laplacians associated to the discrete complexes (3.21) and (3.22). This means that one can obtain compatible finite element spaces for the tensor Laplacian by choosing the underlying finite element 


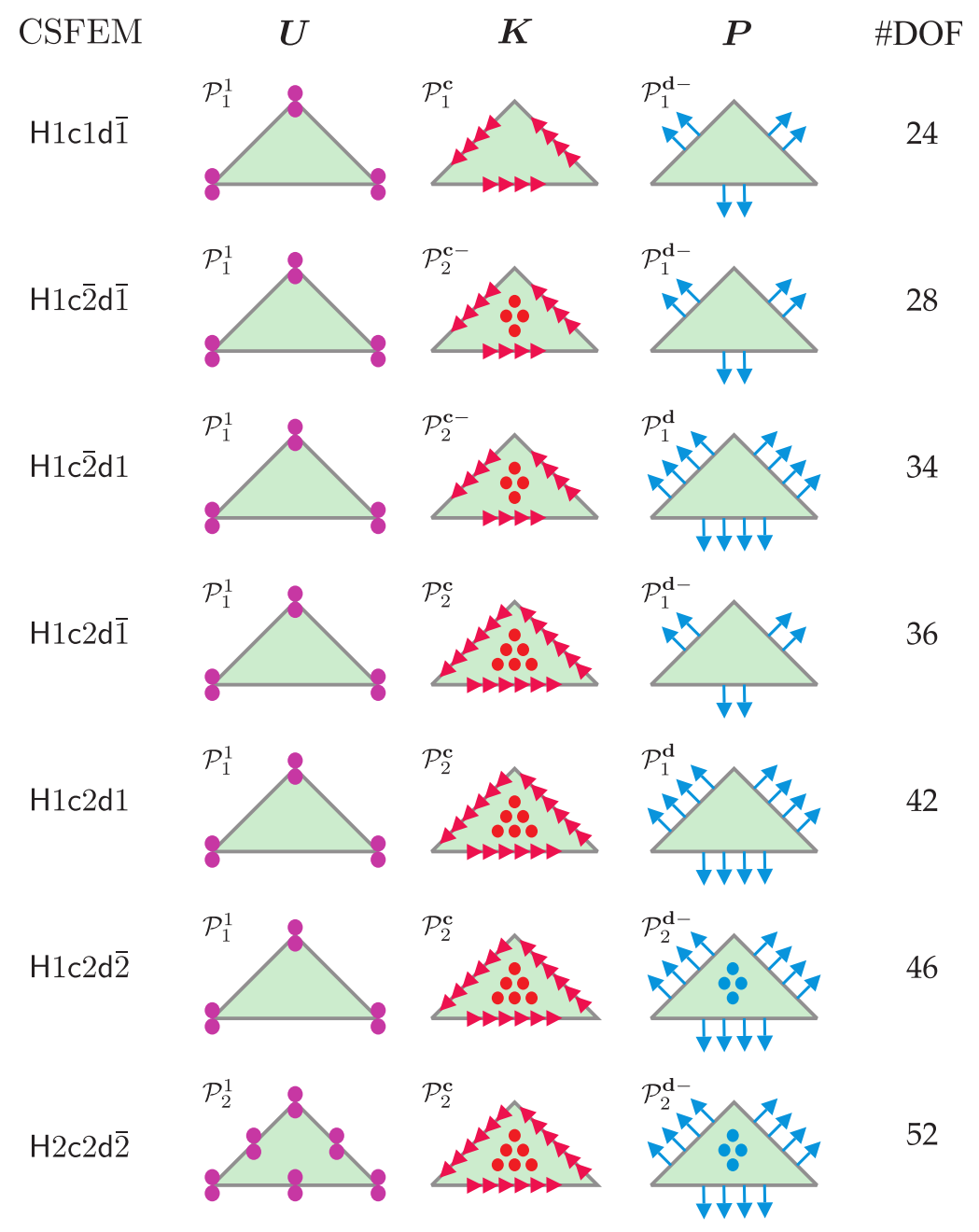

Figure 7: The conventional finite element diagrams for some first-order and second-order CSFEMs. Numerical examples suggest that these 7 combinations have a better performance compared to the other 25 possible first-order and second-order CSFEMs. For each CSFEM, DOF indicates the total number of degrees of freedom per each triangle.

spaces of (3.21) and (3.22) in the order that they appear in the discrete complexes. This is not the case for nonlinear elasticity. For example, Figure 6 shows all the possible choices for obtaining a first-order CSFEM. Our numerical examples suggest that the combination in the third row of Figure 6 that is denoted by $\mathrm{H} 1 \mathrm{c} 1 \mathrm{~d} \overline{1}$ has the best performance among all the first-order combinations. However, the choice of $\left(\boldsymbol{U}_{h}, \boldsymbol{K}_{h}\right) \in \mathcal{P}_{1}^{1}\left(T \mathcal{B}_{h}\right) \times \mathcal{P}_{1}^{\mathbf{c}}\left(\mathcal{B}_{h}\right)$ is not consistent with the order of appearance of the $H^{1}$ - and $H^{\mathbf{c}}$-conformal spaces in the discrete complexes (3.21a) and (3.21b).

Since (3.24) corresponds to a saddle point of a variational problem, the numerical implementation of (3.24) leads to the solution of linear systems with indefinite matrices. It is well-known that standard numerical methods for solving linear systems may have a poor performance when applied to such linear systems and one may need to use methods that are specifically developed for these linear systems, for example see $[43,44]$. Our numerical examples suggest that 7 out of the 32 possible CSFEMs associated to first-order and second-order elements lead to stiffness matrices that can be efficiently solved using standard non-iterative methods. These combinations are given in Figure 7. 

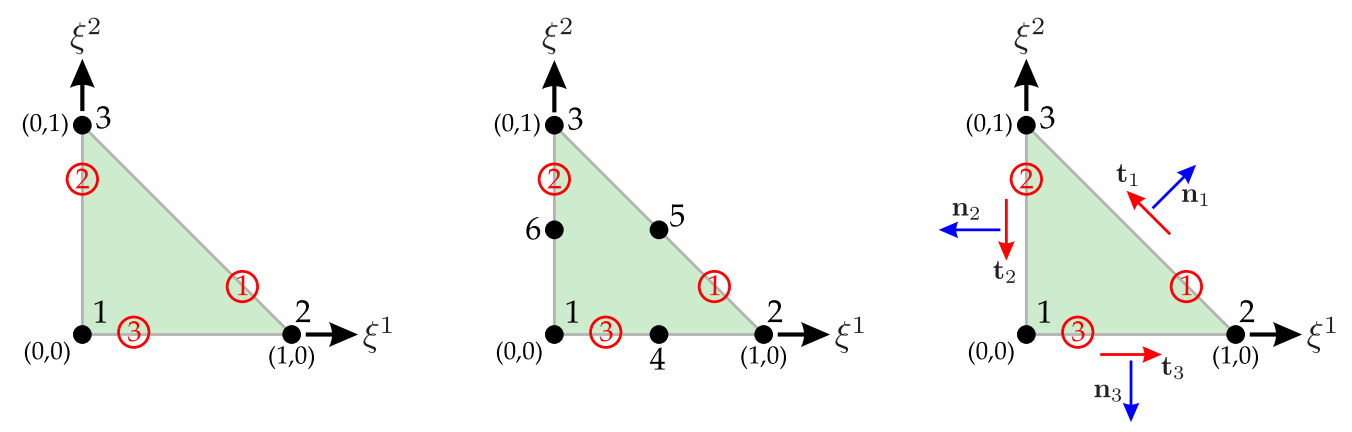

Figure 8: The three-node reference element (left), the six-node reference element (middle), and the reference directions for the unit normal and tangent vectors (right). The circled numbers are the numbering of edges in the reference elements.

\subsection{Implementation}

In this section, we discuss the implementation of CSFEMs. We first use affine transformations between the reference element and an arbitrary element of a mesh, interpolations of the field variables via shape functions, and the assembly process to write the matrix formulation of (3.24). Then, we discuss Newton's method that is used for solving the resulting nonlinear equations. Finally, we discuss the nonlinear constitutive equations used in our numerical examples.

\subsubsection{The Matrix Formulation}

For implementing CSFEMs, it is easier to work with the matrix formulation of the governing equations. Given a second-order tensor $\boldsymbol{T}$, we define its corresponding vector $\lceil\boldsymbol{T}\rceil$ by

$$
\lceil\boldsymbol{T}\rceil:=\left[\begin{array}{llll}
T^{11} & T^{12} & T^{21} & T^{22}
\end{array}\right]^{\top} .
$$

By using the $L^{2}$-inner product of tensor fields and the standard inner product $\langle$, $\left.\rangle\right\rangle$ of the Euclidean space, it is straightforward to show that $\left\langle\langle\boldsymbol{Y}, \boldsymbol{Z}\rangle_{L^{2}}=\left\langle\langle\lceil\boldsymbol{Y}\rceil,\lceil\boldsymbol{Z}\rceil\rangle=\int_{\mathcal{B}}\lceil\boldsymbol{Y}\rceil^{\top}\lceil\boldsymbol{Z}\rceil d A=\int_{\mathcal{B}}\lceil\boldsymbol{Z}\rceil^{\top}\lceil\boldsymbol{Y}\rceil d A\right.\right.$. Using the standard matrix multiplication, (3.24) can be written as

$$
\begin{array}{ll}
\int_{\mathcal{B}_{h}}\lceil\operatorname{grad} \boldsymbol{\Upsilon}\rceil^{\top}\left\lceil\boldsymbol{P}_{h}\right\rceil d A=\int_{\mathcal{B}_{h}} \rho_{0} \boldsymbol{\Upsilon}^{\top} \boldsymbol{B} d A+\int_{\Gamma_{t}} \boldsymbol{\Upsilon}^{\top} \overline{\boldsymbol{T}} d s, & \forall \boldsymbol{\Upsilon} \in \mathcal{P}_{r}^{1}\left(T \mathcal{B}_{h}, \Gamma_{d}\right), \\
\int_{\mathcal{B}_{h}}\lceil\boldsymbol{\kappa}\rceil^{\top}\left\lceil\widetilde{\boldsymbol{P}}\left(\left\lceil\boldsymbol{K}_{h}\right\rceil\right)\right\rceil d A-\int_{\mathcal{B}_{h}}\lceil\boldsymbol{\kappa}\rceil^{\top}\left\lceil\boldsymbol{P}_{h}\right\rceil d A=0, & \forall\lceil\boldsymbol{\kappa}\rceil \in\left\lceil V_{p}^{\mathbf{c}}\right\rceil, \\
\int_{\mathcal{B}_{h}}(\operatorname{div} \boldsymbol{\pi})^{\top} \boldsymbol{U}_{h} d A+\int_{\mathcal{B}_{h}}\lceil\boldsymbol{\pi}\rceil^{\top}\left\lceil\boldsymbol{K}_{h}\right\rceil d A=\int_{\Gamma_{d}}(\boldsymbol{\pi}(\boldsymbol{N}))^{\top} \overline{\boldsymbol{U}} d s, & \forall\lceil\boldsymbol{\pi}\rceil \in\left\lceil V_{q}^{\mathbf{d}}\left(\Gamma_{t}\right)\right\rceil,
\end{array}
$$

where $\left(\boldsymbol{U}_{h},\left\lceil\boldsymbol{K}_{h}\right\rceil,\left\lceil\boldsymbol{P}_{h}\right\rceil\right) \in \mathcal{P}_{r}^{1}\left(T \mathcal{B}_{h}\right) \times\left\lceil V_{p}^{\mathbf{c}}\right\rceil \times\left\lceil V_{q}^{\mathbf{d}}\right\rceil$, with $\left\lceil V_{p}^{\mathbf{c}}\right\rceil,\left\lceil V_{q}^{\mathbf{d}}\right\rceil$, and $\left\lceil V_{q}^{\mathbf{d}}\left(\Gamma_{t}\right)\right\rceil$ denote the corresponding spaces of vectors.

Let $\widehat{\mathcal{T}}$ denote the reference element with coordinates $\boldsymbol{\xi}=\left(\xi^{1}, \xi^{2}\right)$ as shown in Figure 8. Also suppose the hat $\widehat{\cdot}$ on any quantity denotes the corresponding quantity in the reference element. The reference element $\widehat{\mathcal{T}}$ can be mapped onto any $\mathcal{T} \in \mathcal{B}_{h}$ by an affine transformation $\mathbf{T}_{\mathcal{T}}$ given by

$$
\mathbf{T}_{\mathcal{T}}: \widehat{\mathcal{T}} \longrightarrow \mathcal{T}, \quad \mathbf{T}_{\mathcal{T}}(\boldsymbol{\xi}):=\mathbf{J}_{\mathcal{T}} \boldsymbol{\xi}+\mathbf{b}_{\mathcal{T}}
$$

with

$$
\mathbf{J}_{\mathcal{T}}:=\left[\begin{array}{ll}
v_{2}^{x}-v_{1}^{x} & v_{3}^{x}-v_{1}^{x} \\
v_{2}^{y}-v_{1}^{y} & v_{3}^{y}-v_{1}^{y}
\end{array}\right] \quad \text { and } \quad \mathbf{b}_{\mathcal{T}}:=\left[\begin{array}{c}
v_{1}^{x} \\
v_{1}^{y}
\end{array}\right]
$$


where $\left(v_{i}^{x}, v_{i}^{y}\right)$ denotes the Cartesian coordinates of the $i$-th vertex of each element. The mapping (3.26) is bijective and $\mathbf{J}_{\mathcal{T}}$ is invertible.

We use the standard Lagrange finite element for $\mathcal{P}_{r}^{1}\left(T \mathcal{B}_{h}\right)$. For the three-node reference element, the Lagrange shape functions read

$$
\mathrm{N}_{1}^{1}(\boldsymbol{\xi})=1-\xi^{1}-\xi^{2}, \quad \mathbf{N}_{2}^{1}(\boldsymbol{\xi})=\xi^{1}, \quad \mathbf{N}_{3}^{1}(\boldsymbol{\xi})=\xi^{2} .
$$

By using (3.27), the Lagrange shape functions for the six-node reference element can be written as

$$
\mathrm{N}_{i}^{2}(\boldsymbol{\xi})=\mathrm{N}_{i}^{1}(\boldsymbol{\xi})\left(2 \mathrm{~N}_{i}^{1}(\boldsymbol{\xi})-1\right), \quad \mathrm{N}_{3+i}^{2}(\boldsymbol{\xi})=4 \mathrm{~N}_{i}^{1}(\boldsymbol{\xi}) \mathrm{N}_{i+1}^{1}(\boldsymbol{\xi}),
$$

where $i=1,2,3$ and $\mathrm{N}_{4}^{1}=\mathrm{N}_{1}^{1}$. The affine transformation (3.26) implies that

$$
\operatorname{grad} \mathrm{N}_{i}^{r}(\boldsymbol{\xi})=\left(\mathbf{J}_{\mathcal{T}}^{-1}\right)^{\top} \widehat{\operatorname{grad}} \mathrm{N}_{i}^{r}(\boldsymbol{\xi})
$$

where $\widehat{\text { grad }}$ is gradient in the reference element coordinates $\boldsymbol{\xi}=\left(\xi^{1}, \xi^{2}\right)$.

The derivation of the local shape functions for $\mathcal{P}_{r}^{\mathbf{d}}\left(\mathcal{B}_{h}\right), \mathcal{P}_{r}^{\mathbf{d}-}\left(\mathcal{B}_{h}\right), \mathcal{P}_{r}^{\mathbf{c}}\left(\mathcal{B}_{h}\right)$, and $\mathcal{P}_{r}^{\mathbf{c}-}\left(\mathcal{B}_{h}\right), r=1,2$ was explained in Examples 9 and 10. For the implementation purposes, we use the pull-back of the local shape functions by the mapping (3.26). We define the following vector representations for these pull-backs:

$$
\begin{array}{ll}
\mathbf{N}_{\mathcal{T}, k}^{\mathbf{d}}(\boldsymbol{\xi}):=c_{J}^{\mathcal{T}, \varepsilon_{i}}\left\lceil\left(\overline{\boldsymbol{s}}_{I, J}^{\mathcal{T}, \mathcal{E}_{i}} \circ \mathbf{T}_{\mathcal{T}}\right)(\boldsymbol{\xi})\right], & \mathbf{N}_{\mathcal{T}, l}^{\mathbf{d}}(\boldsymbol{\xi}):=\left\lceil\left(s_{I, J}^{\mathcal{T}, \mathcal{T}} \circ \mathbf{T}_{\mathcal{T}}\right)(\boldsymbol{\xi})\right], \\
\mathbf{N}_{\mathcal{T}, k}^{\mathbf{c}}(\boldsymbol{\xi}):=e_{J}^{\mathcal{T}, \varepsilon_{i}}\left\lceil\left(\overline{\boldsymbol{r}}_{I, J}^{\mathcal{T}, \mathcal{E}_{i}} \circ \mathbf{T}_{\mathcal{T}}\right)(\boldsymbol{\xi})\right], & \mathbf{N}_{\mathcal{T}, l}^{\mathbf{c}}(\boldsymbol{\xi}):=\left\lceil\left(\boldsymbol{r}_{I, J}^{\mathcal{T}, \mathcal{T}} \circ \mathbf{T}_{\mathcal{T}}\right)(\boldsymbol{\xi})\right],
\end{array}
$$

where

$$
c_{J}^{\mathcal{T}, \mathcal{E}_{i}}=\left\{\begin{array}{ll}
\left\langle\mathbf{n}_{\mathcal{E}}, \mathbf{n}_{\mathcal{E}_{i}}^{\mathcal{T}}\right\rangle, & \text { if } J \text { is odd, } \\
1, & \text { if } J \text { is even, }
\end{array} \quad \text { and } \quad e_{J}^{\mathcal{T}, \mathcal{E}_{i}}= \begin{cases}\left\langle\mathbf{t}_{\mathcal{E}}, \mathbf{t}_{\mathcal{E}_{i}}^{\mathcal{T}}\right\rangle, & \text { if } J \text { is odd }, \\
1, & \text { if } J \text { is even. }\end{cases}\right.
$$

In the above relations, $\mathbf{n}_{\mathcal{E}}$ and $\mathbf{t}_{\mathcal{E}}$ are, respectively, the unit normal and tangent vectors, which are associated to all edges of all the elements in the mesh. For the edges $\mathcal{E}_{i}$ of an element $\mathcal{T}, \mathbf{n}_{\mathcal{E}_{i}}^{\mathcal{T}}$ is the unit outward normal vector and $\mathbf{t}_{\mathcal{E}_{i}}^{\mathcal{T}}$ is the unit tangent vector with counterclockwise orientation. The numbering and the directions of these local vectors must be compatible with the reference element $\widehat{\mathcal{T}}$ (see Figure 8). Note that the subscripts $\mathcal{E}$ and $\mathcal{E}_{i}$ indicate the global numbering and the local numbering of the same edge in the mesh, respectively. The subscripts $k$ and $l$ in (3.30) correspond to the degrees of freedom and the corresponding shape functions in each element. We define

$$
k:=I+2(J-1)+2(i-1) \max J, \quad l:=I+2(J-1)+\max k,
$$

where $i=1,2,3$, and $I=1,2$. For $\mathcal{P}_{1}^{\mathbf{d}-}\left(\mathcal{B}_{h}\right)$ and $\mathcal{P}_{1}^{\mathbf{c}-}\left(\mathcal{B}_{h}\right)$, we have $J=1$. For $\mathcal{P}_{1}^{\mathbf{d}}\left(\mathcal{B}_{h}\right), \mathcal{P}_{1}^{\mathbf{c}}\left(\mathcal{B}_{h}\right)$, $\mathcal{P}_{2}^{\mathbf{d}-}\left(\mathcal{B}_{h}\right)$, and $\mathcal{P}_{2}^{\mathbf{c}-}\left(\mathcal{B}_{h}\right)$, we have $J=1,2$, and $J=1,2,3$ for $\mathcal{P}_{2}^{\mathbf{d}}\left(\mathcal{B}_{h}\right)$ and $\mathcal{P}_{2}^{\mathbf{c}}\left(\mathcal{B}_{h}\right)$. Note that in (3.30), $\mathbf{N}_{\mathcal{T}, l}^{\mathrm{d}}$ and $\mathbf{N}_{\mathcal{T}, l}^{\mathbf{c}}$ are defined only for second-order finite element spaces.

Remark 12. We write the shape functions of $H^{\mathbf{d}}$ and $H^{\mathbf{c}}$ finite element spaces directly in the global coordinates $\boldsymbol{X}=(X, Y)$ (see the Examples 9 and 10). It is also possible to define these shape functions in the reference element and then map them to the global coordinates. For a standard Lagrange shape function, we have $\mathbf{N}(\boldsymbol{X})=\left(\mathbf{N} \circ \mathbf{T}_{\mathcal{T}}^{-1}\right)(\boldsymbol{\xi})$. However, this mapping is not valid for $H^{\mathbf{d}}$ and $H^{\mathbf{c}}$-spaces. In these cases, one should use mappings similar to the Piola mapping for Raviart-Thomas and Nédélec 
elements discussed in $[26, \S 1.4]$.

On each element $\mathcal{T}$ we have

$$
\begin{array}{lll}
\boldsymbol{U}_{\mathcal{T}}=\mathbf{B}^{1} \mathbf{q}_{\mathcal{T}}^{1}, & \left\lceil\boldsymbol{K}_{\mathcal{T}}\right\rceil=\mathbf{B}_{\mathcal{T}}^{\mathbf{c}} \mathbf{q}_{\mathcal{T}}^{\mathbf{c}}, & \left\lceil\boldsymbol{P}_{\mathcal{T}}\right\rceil=\mathbf{B}_{\mathcal{T}}^{\mathbf{d}} \mathbf{q}_{\mathcal{T}}^{\mathbf{d}}, \\
\boldsymbol{\Upsilon}_{\mathcal{T}}=\mathbf{B}^{1} \mathbf{t}_{\mathcal{T}}^{1}, & \left\lceil\boldsymbol{\kappa}_{\mathcal{T}}\right\rceil=\mathbf{B}_{\mathcal{T}}^{\mathbf{c}} \mathbf{t}_{\mathcal{T}}^{\mathbf{c}}, & \left\lceil\boldsymbol{\pi}_{\mathcal{T}}\right\rceil=\mathbf{B}_{\mathcal{T}}^{\mathbf{d}} \mathbf{t}_{\mathcal{T}}^{\mathbf{d}},
\end{array}
$$

where $\mathbf{q}_{\mathcal{T}}^{1}, \mathbf{q}_{\mathcal{T}}^{\mathbf{c}}$, and $\mathbf{q}_{\mathcal{T}}^{\mathbf{d}}$ denote the local vectors of unknown degrees of freedom corresponding to displacement, displacement gradient, and stress, respectively. These vectors are written as

$$
\begin{aligned}
& \mathbf{q}_{\mathcal{T}}^{1}=\left[\begin{array}{llll}
\mathbf{q}_{\mathcal{T}, 1}^{1} & \mathbf{q}_{\mathcal{T}, 2}^{1} & \cdots & \mathbf{q}_{\mathcal{T}, n^{1}}^{1}
\end{array}\right]^{\top}, \quad \mathbf{q}_{\mathcal{T}, m}^{1}=U_{h}^{J}\left(v_{i}^{x}, v_{i}^{y}\right), \\
& \mathbf{q}_{\mathcal{T}}^{\mathbf{c}}=\left[\begin{array}{llll}
\mathbf{q}_{\mathcal{T}, 1}^{\mathbf{c}} & \mathbf{q}_{\mathcal{T}, 2}^{\mathbf{c}} & \cdots & \mathbf{q}_{\mathcal{T}, n^{c}}^{\mathbf{c}}
\end{array}\right]^{\top}, \quad \mathbf{q}_{\mathcal{T}, k}^{\mathbf{c}}=\bar{\phi}_{I, J}^{\mathcal{T}, \mathcal{E}_{i}}, \quad \mathbf{q}_{\mathcal{T}, l}^{\mathbf{c}}=\phi_{I, J}^{\mathcal{T}, \mathcal{T}}, \\
& \mathbf{q}_{\mathcal{T}}^{\mathbf{d}}=\left[\begin{array}{llll}
\mathbf{q}_{\mathcal{T}, 1}^{\mathbf{d}} & \mathbf{q}_{\mathcal{T}, 2}^{\mathbf{d}} & \cdots & \mathbf{q}_{\mathcal{T}, n^{d}}^{\mathbf{d}}
\end{array}\right]^{\top}, \quad \mathbf{q}_{\mathcal{T}, k}^{\mathbf{d}}=\bar{\psi}_{I, J}^{\mathcal{T}, \varepsilon_{i}}, \quad \mathbf{q}_{\mathcal{T}, l}^{\mathbf{d}}=\psi_{I, J}^{\mathcal{T}, \mathcal{T}} .
\end{aligned}
$$

In (3.33a), $U_{h}^{J}\left(v_{i}^{x}, v_{i}^{y}\right), J=1,2$, is the value of the displacement at the $i$-th node of each element. We define $m:=J+2(i-1)$. In $(3.33 \mathrm{~b})$ and $(3.33 \mathrm{c}), \bar{\phi}_{I, J}^{\mathcal{T}, \mathcal{E}_{i}}, \phi_{I, J}^{\mathcal{T}, \mathcal{T}}$ and $\bar{\psi}_{I, J}^{\mathcal{T}, \mathcal{E}_{i}}, \psi_{I, J}^{\mathcal{T}, \mathcal{T}}$ have been given in Example 10 and 9, respectively. The subscripts $k$ and $l$ are defined in (3.31). The size of the vectors $\left(\mathbf{q}_{\mathcal{T}}^{1}, \mathbf{q}_{\mathcal{T}}^{\mathbf{c}}, \mathbf{q}_{\mathcal{T}}^{\mathbf{d}}\right)$ is denoted by $\left(n^{1}, n^{c}, n^{d}\right)$, which depends on the choice of CSFEMs. These values are given in Figure 7 for the first-order and the second-order CSFEMs. For example, for $\mathrm{H} 1 \mathrm{cld} \overline{1}$ we have $\left(n^{1}, n^{c}, n^{d}\right)=(6,12,6)$, and for $\mathrm{H} 2 \mathrm{c} 2 \mathrm{~d} 2$ we have $\left(n^{1}, n^{c}, n^{d}\right)=(12,24,16)$. The vectors $\mathbf{t}_{\mathcal{T}}^{1}$, $\mathbf{t}_{\mathcal{T}}^{\mathbf{c}}$, and $\mathbf{t}_{\mathcal{T}}^{\mathbf{d}}$ in (3.32) are arbitrary vectors associated with the corresponding test spaces. Moreover, by using (3.27), (3.28), (3.30), and (3.31), the sparse matrices $\mathbf{B}_{\mathcal{T}}^{1}$, $\mathbf{B}_{\mathcal{T}}^{\mathbf{c}}$, and $\mathbf{B}_{\mathcal{T}}^{\mathbf{d}}$ in (3.32) can be written as

$$
\begin{aligned}
\mathbf{B}^{1} & =\left[\begin{array}{ccccccc}
\mathbf{N}_{1}^{r} & 0 & \mathbf{N}_{2}^{r} & 0 & \cdots & \mathbf{N}_{n^{1}}^{r} & 0 \\
0 & \mathbf{N}_{1}^{r} & 0 & \mathbf{N}_{2}^{r} & \cdots & 0 & \mathbf{N}_{n^{1}}^{r}
\end{array}\right]_{2 \times n^{1}}, \\
\mathbf{B}_{\mathcal{T}}^{\mathbf{c}} & =\left[\begin{array}{lllll}
\mathbf{N}_{\mathcal{T}, 1}^{\mathbf{c}} & \mathbf{N}_{\mathcal{T}, 2}^{\mathbf{c}} & \cdots & \mathbf{N}_{\mathcal{T}, n^{c}}^{\mathbf{c}}
\end{array}\right]_{4 \times n^{c}}, \\
\mathbf{B}_{\mathcal{T}}^{\mathbf{d}} & =\left[\begin{array}{llll}
\mathbf{N}_{\mathcal{T}, 1}^{\mathbf{d}} & \mathbf{N}_{\mathcal{T}, 1}^{\mathbf{d}} & \cdots & \mathbf{N}_{\mathcal{T}, n^{d}}^{\mathbf{d}}
\end{array}\right]_{4 \times n^{d}} .
\end{aligned}
$$

The remaining terms of the left-hand sides of (3.25) are interpolated as

$$
\left\lceil\operatorname{grad} \Upsilon_{\mathcal{T}}\right\rceil=\mathbf{G}_{\mathcal{T}}^{1} \mathbf{t}_{\mathcal{T}}^{1}, \quad \text { and } \quad \operatorname{div} \boldsymbol{\pi}_{\mathcal{T}}=\mathbf{D}_{\mathcal{T}}^{\mathrm{d}} \mathbf{t}_{\mathcal{T}}^{\mathrm{d}},
$$

where the sparse matrices $\mathbf{G}_{\mathcal{T}}^{1}$ and $\mathbf{D}_{\mathcal{T}}^{\mathbf{d}}$ are given by

$$
\begin{aligned}
& \mathbf{G}_{\mathcal{T}}^{1}=\left[\begin{array}{ccccccc}
\operatorname{gradN} N_{1}^{r} & \mathbf{0} & \operatorname{gradN}_{2}^{r} & \mathbf{0} & \cdots & \operatorname{gradN}_{n^{1}}^{r} & \mathbf{0} \\
\mathbf{0} & \operatorname{gradN}_{1}^{r} & \mathbf{0} & \operatorname{gradN}_{2}^{r} & \cdots & \mathbf{0} & \operatorname{grad} N_{n^{1}}^{r}
\end{array}\right]_{4 \times n^{1}}, \\
& \mathbf{D}_{\mathcal{T}}^{\mathbf{d}}=\left[\begin{array}{lllll}
\operatorname{div} \mathbf{N}_{\mathcal{T}, 1}^{\mathbf{d}} & \operatorname{div} \mathbf{N}_{\mathcal{T}, 2}^{\mathbf{d}} & \cdots & \operatorname{div} \mathbf{N}_{\mathcal{T}, n^{d}}^{\mathbf{d}}
\end{array}\right]_{2 \times n^{d}} .
\end{aligned}
$$

We use (3.29) to obtain $\mathbf{G}_{\mathcal{T}}^{1}$. For calculating $\mathbf{D}_{\mathcal{T}}^{\mathbf{d}}$, we use the following relations using (3.30a) and (3.31):

$$
\operatorname{div} \mathbf{N}_{\mathcal{T}, k}^{\mathrm{d}}(\boldsymbol{\xi})=c_{J}^{\mathcal{T}, \mathcal{E}_{i}}\left\lceil\left(\operatorname{div} \boldsymbol{s}_{I, J}^{\mathcal{T}, \mathcal{E}_{i}} \circ \mathbf{T}_{\mathcal{T}}\right)(\boldsymbol{\xi})\right], \quad \operatorname{div} \mathbf{N}_{\mathcal{T}, l}^{\mathrm{d}}(\boldsymbol{\xi})=\left\lceil\left(\operatorname{div} \boldsymbol{s}_{I, J}^{\mathcal{T}, \mathcal{T}} \circ \mathbf{T}_{\mathcal{T}}\right)(\boldsymbol{\xi})\right\rceil
$$

The equations (3.25) together with (3.26), (3.34), and (3.36), allows one to define the following 
local stiffness matrices:

$$
\begin{array}{ll}
\mathbf{K}_{\mathcal{T}}^{1 \mathbf{d}}=\operatorname{det} \mathbf{J}_{\mathcal{T}} \int_{\widehat{\mathcal{T}}}\left(\mathbf{G}_{\mathcal{T}}^{1}\right)^{\top} \mathbf{B}_{\mathcal{T}}^{\mathbf{d}} d \widehat{A}, & \mathbf{K}_{\mathcal{T}}^{\mathbf{c d}}=-\operatorname{det} \mathbf{J}_{\mathcal{T}} \int_{\widehat{\mathcal{T}}}\left(\mathbf{B}_{\mathcal{T}}^{\mathbf{c}}\right)^{\top} \mathbf{B}_{\mathcal{T}}^{\mathbf{d}} d \widehat{A}, \\
\mathbf{K}_{\mathcal{T}}^{\mathbf{d} 1}=\operatorname{det} \mathbf{J}_{\mathcal{T}} \int_{\widehat{\mathcal{T}}}\left(\mathbf{D}_{\mathcal{T}}^{\mathbf{d}}\right)^{\top} \mathbf{B}^{1} d \widehat{A}, & \mathbf{K}_{\mathcal{T}}^{\mathbf{d c}}=\operatorname{det} \mathbf{J}_{\mathcal{T}} \int_{\widehat{\mathcal{T}}}\left(\mathbf{B}_{\mathcal{T}}^{\mathbf{d}}\right)^{\top} \mathbf{B}_{\mathcal{T}}^{\mathbf{c}} d \widehat{A} .
\end{array}
$$

Note that $\mathbf{K}_{\mathcal{T}}^{\text {dc }}=-\left(\mathbf{K}_{\mathcal{T}}^{\text {cd }}\right)^{\top}$. The nonlinear part of (3.25), i.e., the first term on the left-hand side of the second equation, which is associated with the constitutive relation, has the following matrix representation

$$
\mathbf{S}_{\mathcal{T}}^{\mathbf{c}}\left(\mathbf{q}_{\mathcal{T}}^{\mathbf{c}}\right)=\operatorname{det} \mathbf{J}_{\mathcal{T}} \int_{\widehat{\mathcal{T}}}\left(\mathbf{B}_{\mathcal{T}}^{\mathbf{c}}\right)^{\top}\left\lceil\widetilde{\boldsymbol{P}}\left(\mathbf{B}_{\mathcal{T}}^{\mathbf{c}} \mathbf{q}_{\mathcal{T}}^{\mathbf{c}}\right)\right\rceil d \widehat{A}
$$

The term containing the body force in (3.25) gives the following vector

$$
\mathbf{F}_{\mathcal{T}}^{1}=\rho_{0} \operatorname{det} \mathbf{J}_{\mathcal{T}} \int_{\widehat{\mathcal{T}}}\left(\mathbf{B}^{1}\right)^{\top}\left(\boldsymbol{B} \circ \mathbf{T}_{\mathcal{T}}\right) d \widehat{A}
$$

The terms in (3.25) that contain the boundary traction and the boundary displacement result in the following vectors:

$$
\mathbf{F}_{\varepsilon_{t}}^{1}=\ell_{\varepsilon_{t}} \int_{\widehat{\mathcal{\varepsilon}}}\left(\mathbf{B}_{\varepsilon_{t}}^{1}\right)^{\boldsymbol{T}}\left(\overline{\boldsymbol{T}} \circ \mathbf{T}_{\mathcal{T}}\right) d \widehat{s}, \quad \text { and } \quad \mathbf{F}_{\varepsilon_{d}}^{\mathbf{d}}=\ell_{\varepsilon_{d}} \int_{\widehat{\mathcal{E}}}\left(\mathbf{B}_{\varepsilon_{d}}^{\mathbf{d}}\right)^{\boldsymbol{T}}\left(\overline{\boldsymbol{U}} \circ \mathbf{T}_{\mathcal{T}}\right) d \widehat{s}
$$

where $\varepsilon_{t}$ and $\varepsilon_{d}$ are those edges of elements that lie on the traction and displacement boundaries, respectively. $\ell_{\varepsilon_{t}}$ and $\ell_{\varepsilon_{d}}$ denote the length of $\mathcal{E}_{t}$ and $\mathcal{E}_{d}$, respectively. The matrices $\mathbf{B}_{\varepsilon_{t}}^{1}$ and $\mathbf{B}_{\mathcal{E}_{d}}^{\mathrm{d}}$ are defined similarly to (3.34) only for the edges at the boundary. Finally, (3.33), (3.37), (3.38), (3.39), and (3.40) allow one to write (3.25) as

$$
\begin{aligned}
& \sum_{\mathcal{T} \in \Delta_{2}\left(\mathcal{B}_{h}\right)}\left(\mathbf{t}_{\mathcal{T}}^{1}\right)^{\top}\left(\mathbf{K}_{\mathcal{T}}^{1 \mathbf{d}} \mathbf{q}_{\mathcal{T}}^{\mathbf{d}}-\mathbf{F}_{\mathcal{T}}^{1}-\mathbf{F}_{\varepsilon_{t}}^{1}\right)=0, \\
& \sum_{\mathcal{T} \in \Delta_{2}\left(\mathcal{B}_{h}\right)}\left(\mathbf{t}_{\mathcal{T}}^{\mathbf{c}}\right)^{\top}\left(\mathbf{S}_{\mathcal{T}}^{\mathbf{c}}\left(\mathbf{q}_{\mathcal{T}}^{\mathbf{c}}\right)+\mathbf{K}_{\mathcal{T}}^{\mathbf{c d}} \mathbf{q}_{\mathcal{T}}^{\mathbf{d}}\right)=0, \\
& \sum_{\mathcal{T} \in \Delta_{2}\left(\mathcal{B}_{h}\right)}\left(\mathbf{t}_{\mathcal{T}}^{\mathbf{d}}\right)^{\top}\left(\mathbf{K}_{\mathcal{T}}^{\mathbf{d} 1} \mathbf{q}_{\mathcal{T}}^{1}+\mathbf{K}_{\mathcal{T}}^{\mathbf{d c}} \mathbf{q}_{\mathcal{T}}^{\mathbf{c}}-\mathbf{F}_{\varepsilon_{d}}^{\mathbf{d}}\right)=0 .
\end{aligned}
$$

By assembling the local matrices (3.33), (3.37), (3.38), (3.39), and (3.40), one obtains

$$
\begin{aligned}
\left\{\mathbf{q}_{\mathcal{B}_{h}}^{1}, \mathbf{q}_{\mathcal{B}_{h}}^{\mathbf{c}}, \mathbf{q}_{\mathcal{B}_{h}}^{\mathbf{d}}, \mathbf{t}_{\mathcal{B}_{h}}^{1}, \mathbf{t}_{\mathcal{B}_{h}}^{\mathbf{c}}, \mathbf{t}_{\mathcal{B}_{h}}^{\mathbf{d}}\right\}=\underset{\mathcal{T} \in \Delta_{2}\left(\mathcal{B}_{h}\right)}{\overline{\mathbf{A}}}\left\{\mathbf{q}_{\mathcal{T}}^{1}, \mathbf{q}_{\mathcal{T}}^{\mathbf{c}}, \mathbf{q}_{\mathcal{T}}^{\mathbf{d}}, \mathbf{t}_{\mathcal{T}}^{1}, \mathbf{t}_{\mathcal{T}}^{\mathbf{c}}, \mathbf{t}_{\mathcal{T}}^{\mathbf{d}}\right\}, \\
\left\{\mathbf{K}_{\mathcal{B}_{h}}^{1 \mathbf{d}}, \mathbf{K}_{\mathcal{B}_{h}}^{\mathbf{d} 1}, \mathbf{K}_{\mathcal{B}_{h}}^{\mathbf{c d}}, \mathbf{K}_{\mathcal{B}_{h}}^{\mathbf{d c}}, \mathbf{S}_{\mathcal{B}_{h}}^{\mathbf{c}}, \mathbf{F}_{\mathcal{B}_{h}}^{1}, \mathbf{F}_{\Gamma_{t}}^{1}, \mathbf{F}_{\Gamma_{d}}^{\mathbf{d}}\right\}=\underset{\mathcal{T} \in \Delta_{2}\left(\mathcal{B}_{h}\right)}{\mathbf{A}}\left\{\mathbf{K}_{\mathcal{T}}^{1 \mathbf{d}}, \mathbf{K}_{\mathcal{T}}^{\mathbf{d} 1}, \mathbf{K}_{\mathcal{T}}^{\mathbf{c d}}, \mathbf{K}_{\mathcal{T}}^{\mathbf{d c}}, \mathbf{S}_{\mathcal{T}}^{\mathbf{c}}, \mathbf{F}_{\mathcal{T}}^{1}, \mathbf{F}_{\mathcal{E}_{t}}^{1}, \mathbf{F}_{\mathcal{E}_{d}}^{\mathbf{d}}\right\},
\end{aligned}
$$

where $\overline{\mathbf{A}}$ simply concatenates all the column vectors of local degrees of freedom vertically to a global vector and $\mathbf{A}$ denotes the assembly operator of local matrices. Using the assembled matrices, one can define

$$
\mathbb{Q}=\left[\begin{array}{l}
\mathbf{q}_{\mathcal{B}_{h}}^{1} \\
\mathbf{q}_{\mathcal{B}_{h}}^{\mathbf{c}} \\
\mathbf{q}_{\mathcal{B}_{h}}^{\mathbf{d}_{h}}
\end{array}\right], \quad \text { and } \quad \mathbb{T}=\left[\begin{array}{l}
\mathbf{t}_{\mathcal{B}_{h}}^{1} \\
\mathbf{t}_{\mathcal{B}_{h}}^{\mathbf{c}} \\
\mathbf{t}_{\mathcal{B}_{h}}^{\mathbf{d}_{h}}
\end{array}\right]
$$

where the vector $\mathbb{Q}$ includes all the unknown degrees of freedom of the system and $\mathbb{T}$ is an arbitrary 
vector corresponding to the test fields. The global stiffness matrix, the global nonlinear stiffness part of the system, and the global external forces can be written as

$$
\mathbb{K}=\left[\begin{array}{ccc}
\mathbf{0} & \mathbf{0} & \mathbf{K}_{\mathcal{B}_{h}}^{1 \mathbf{d}} \\
\mathbf{0} & \mathbf{0} & \mathbf{K}_{\mathcal{B}_{h}}^{\mathbf{c d}} \\
\mathbf{K}_{\mathcal{B}_{h}}^{\mathbf{d} 1} & \mathbf{K}_{\mathcal{B}_{h}}^{\mathbf{d c}} & \mathbf{0}
\end{array}\right], \quad \mathbb{S}(\mathbb{Q})=\left[\begin{array}{c}
\mathbf{0} \\
\mathbf{S}_{\mathcal{B}_{h}}^{\mathbf{c}}\left(\mathbf{q}_{\mathcal{B}_{h}}^{\mathbf{c}}\right) \\
\mathbf{0}
\end{array}\right], \quad \mathbb{F}=\left[\begin{array}{c}
\mathbf{F}_{\mathcal{B}_{h}}^{1}+\mathbf{F}_{\Gamma_{t}}^{1} \\
\mathbf{0} \\
\mathbf{F}_{\Gamma_{d}}^{\mathbf{d}}
\end{array}\right]
$$

Let $n_{\mathcal{V}}$ and $n_{\mathcal{E}}$ be the total numbers of vertices and edges in the mesh, respectively. The total number of degrees of freedom is $n_{\mathcal{D}}=n^{1} n_{\mathcal{V}}+\left(n^{c}+n^{d}\right) n_{\mathcal{E}}$, where $n^{1}, n^{c}$, and $n^{d}$ depend on the specific choice of CSFEMs. Therefore, the size of the sparse matrix $\mathbb{K}$ is $n_{\mathcal{D}} \times n_{\mathcal{D}}$, and the size of the vectors $\mathbb{T}, \mathbb{Q}$, $\mathbb{S}$, and, $\mathbb{F}$ is $n_{\mathcal{D}} \times 1$. Finally, by using (3.42) and (3.43), (3.41) can be written as

$$
\mathbb{T}^{\top}(\mathbb{K} \mathbb{Q}+\mathbb{S}(\mathbb{Q})-\mathbb{F})=0
$$

Since $\mathbb{T}$ is arbitrary, one concludes that

$$
\mathbb{K} \mathbb{Q}+\mathbb{S}(\mathbb{Q})=\mathbb{F}
$$

\subsubsection{Newton's Method and the Tangent Stiffness Matrix}

Let $\mathbb{V}=\mathbb{K} \mathbb{Q}+\mathbb{S}(\mathbb{Q})-\mathbb{F}$ be the residual of the nonlinear equation (3.44) for some initial guess. By using Newton's method, the solution of (3.44) can be obtained iteratively as $\mathbb{Q}_{i+1}=\mathbb{Q}_{i}-\mathbb{K}_{t}^{-1}\left(\mathbb{Q}_{i}\right) \mathbb{V}\left(\mathbb{Q}_{i}\right)$, where $i$ is the iteration number and the tangent stiffness matrix $\mathbb{K}_{t}$ is given by

$$
\mathbb{K}_{t}=\left[\begin{array}{ccc}
\mathbf{0} & \mathbf{0} & \mathbf{K}_{\mathcal{B}_{h}}^{1 \mathbf{d}} \\
\mathbf{0} & \mathbf{H}_{\mathcal{B}_{h}}^{\text {cc }} & \mathbf{K}_{\mathcal{B}_{h}}^{\text {cd }} \\
\mathbf{K}_{\mathcal{B}_{h}}^{\text {d1 }} & \mathbf{K}_{\mathcal{B}_{h}}^{\text {dc }} & \mathbf{0}
\end{array}\right]
$$

Here, $\mathbf{H}_{\mathcal{B}_{h}}^{\mathbf{c c}}$ is obtained by assembling $\mathbf{H}_{\mathcal{T}}^{\mathbf{c c}}=\operatorname{det} \mathbf{J}_{\mathcal{T}} \int_{\widehat{\mathcal{T}}}\left(\mathbf{B}_{\mathcal{T}}^{\mathbf{c}}\right)^{\top} \widetilde{\mathbf{C}}_{\mathcal{T}} \mathbf{B}_{\mathcal{T}}^{\mathbf{c}} d \widehat{A}$ for all $\mathcal{T} \in \Delta_{2}\left(\mathcal{B}_{h}\right)$.

Remark 13. The tangent stiffness matrix $\mathbb{K}_{t}$ is non-symmetric and indefinite, in general. As we mentioned in $\S 3.2$, the linear system associated to $\mathbb{K}_{t}$ can be efficiently solved using standard noniterative methods for 7 out of the 32 first-order and second-order CSFEMs (see Figure 7). Also note that if one does not apply Green's formula to (2.6) and use this equation instead of (2.7) in the mixed formulation (2.8), then the corresponding tangent stiffness matrix will be symmetric.

\subsubsection{Constitutive Equations}

For our numerical examples in the next section, we consider compressible neo-Hookean materials with the energy functions $W_{1}(\boldsymbol{F})$ and $W_{2}(\boldsymbol{F})$, and Ogden materials with the energy function $W_{3}(\boldsymbol{F})$, given as

$$
\begin{aligned}
& W_{1}(\boldsymbol{F})=\frac{\mu}{2}\left(I_{1}-3\right)-\frac{\mu}{2} \ln I_{3}+\frac{\kappa}{2}\left(I_{3}^{1 / 2}-1\right)^{2}, \\
& W_{2}(\boldsymbol{F})=\frac{\mu}{2}\left(I_{1}-3\right)-\frac{\mu}{2} \ln I_{3}+\frac{\kappa}{8}\left(\ln I_{3}\right)^{2} \\
& W_{3}(\boldsymbol{F})=\sum_{i=1}^{m} \frac{\mu_{i}}{\alpha_{i}}\left(\lambda_{1}^{\alpha_{i}}+\lambda_{2}^{\alpha_{i}}+\lambda_{3}^{\alpha_{i}}-3\right)+\frac{\kappa}{2}\left(I_{3}^{1 / 2}-1\right)^{2},
\end{aligned}
$$

where $\mu$ and $\kappa$ are the shear and bulk moduli for infinitesimal strains, respectively. Also, $\boldsymbol{F}:=\boldsymbol{I}+\boldsymbol{K}$ is the deformation gradient, and $I_{i}, i=1,2,3$, are the principal invariants of the right Cauchy-Green 
deformation tensor $\boldsymbol{C}=\boldsymbol{F}^{\top} \boldsymbol{F}$, where $I_{1}=\operatorname{tr}(\boldsymbol{C})$ and $I_{3}=\operatorname{det} \boldsymbol{C}$. In (3.45c), $\lambda_{i}, i=1,2,3$, are the principal stretches and $\mu_{i}$ 's and $\alpha_{i}$ 's are some material constants.

Using (3.45), the constitutive relations read

$$
\begin{aligned}
& \widetilde{\boldsymbol{P}}_{1}(\boldsymbol{F})=\mu\left(\boldsymbol{F}-\boldsymbol{F}^{-\mathrm{T}}\right)+\kappa\left(J^{2}-J\right) \boldsymbol{F}^{-\mathrm{T}} \\
& \widetilde{\boldsymbol{P}}_{2}(\boldsymbol{F})=\mu\left(\boldsymbol{F}-\boldsymbol{F}^{-\mathrm{T}}\right)+\kappa(\ln J) \boldsymbol{F}^{-\mathrm{T}} \\
& \widetilde{\boldsymbol{P}}_{3}(\boldsymbol{F})=\sum_{i=1}^{m} \mu_{i} J^{-\frac{\alpha_{i}}{3}} \boldsymbol{F}^{-\mathrm{T}}\left(-\frac{1}{3} \operatorname{tr}\left(\boldsymbol{C}^{\frac{\alpha_{i}}{2}}\right) \boldsymbol{I}+\boldsymbol{C}^{\frac{\alpha_{i}}{2}}\right)+\kappa\left(J^{2}-J\right) \boldsymbol{F}^{-\mathrm{T}},
\end{aligned}
$$

where $J=\operatorname{det} \boldsymbol{F}$, and $\boldsymbol{F}^{-\top}:=\left(\boldsymbol{F}^{-1}\right)^{\top}$.

For the neo-Hookean materials (3.45a) and (3.45b), and for the 2D case, the elasticity tensors have the following matrix representations

$$
\begin{aligned}
& \widetilde{\mathbf{C}}_{1}(\boldsymbol{F})=\mu \mathbf{I}+\left(\frac{\mu}{J}-\kappa(J-1)\right) \overline{\mathbf{I}}+\left(\mu+\kappa J^{2}\right)\left\lceil\boldsymbol{F}^{-\mathrm{T}}\right\rceil\left\lceil\boldsymbol{F}^{-\mathrm{T}}\right\rceil^{\mathbf{T}}, \\
& \widetilde{\mathbf{C}}_{2}(\boldsymbol{F})=\mu \mathbf{I}+\frac{\mu-\kappa \ln J}{J} \overline{\mathbf{I}}+(\mu+\kappa(1-\ln J))\left\lceil\boldsymbol{F}^{-\mathrm{T}}\right\rceil\left\lceil\boldsymbol{F}^{-\mathrm{T}}\right\rceil^{\mathbf{T}},
\end{aligned}
$$

where $\mathbf{I}$ is the $4 \times 4$ identity matrix, and $\mathbf{I}$ denotes an anti-diagonal matrix with non-zero components $\overline{\mathrm{I}}^{14}=\overline{\mathrm{I}}^{41}=-1$ and $\overline{\mathrm{I}}^{23}=\overline{\mathrm{I}}^{32}=1$. The above relations are used for calculating the tangent stiffness matrix. We compute the elasticity tensor of (3.45c) numerically.

\section{Numerical Examples}

To test the performance of CSFEMs introduced in this paper, we consider six different numerical examples in this section.

\subsection{Example 1: Shearing Plate}

As the first example, following [22], we consider a clamped square plate subject to shear loads. The side length of the plate is $1 \mathrm{~mm}$ and we assume the following displacement field (see Figure 9):

$$
\boldsymbol{U}_{e}=\left[\begin{array}{c}
\frac{1}{2} Y^{3}+\frac{1}{2} \sin \left(\frac{\pi Y}{2}\right) \\
0
\end{array}\right]
$$

We assume a neo-Hookean material with energy function $W_{1}(\boldsymbol{F})$ given in $(3.45 \mathrm{a})$, with $\mu=\kappa=$ $1 \mathrm{~N} / \mathrm{mm}^{2}$. Using $\boldsymbol{U}_{e}$, it is straightforward to calculate the deformation gradient $\boldsymbol{F}_{e}$, the first PiolaKirchhoff stress tensor $\boldsymbol{P}_{e}$, the body force, and the boundary traction. Then, $\left(\boldsymbol{U}_{e}, \boldsymbol{F}_{e}, \boldsymbol{P}_{e}\right)$ can be considered as the exact solution of the square plate clamped on the bottom and subject to shear loads on the other sides as shown in Figure 9.

As we mentioned in $§ 3.2$, there are 32 possible first-order and second-order CSFEMs. Our numerical experiments suggest that the 7 combinations shown in Figure 7 have a better performance among all the first-order and second-order CSFEMs. All these 7 combinations pass the patch test in the sense discussed in Hughes [45, §4.6]. For the meshes shown in Figure 9, the $L^{2}$-norm of errors associated to each of these CSFEMs are given in Table 3. Note that the $L^{2}$-norm of a second-order tensor $\boldsymbol{T}$ is 
given by

$$
\|\boldsymbol{T}\|_{L^{2}}^{2}:=\int_{\mathcal{B}}\|\boldsymbol{T}\|^{2} d A, \text { with }\|\boldsymbol{T}\|^{2}:=\sum_{I, J} T^{I J} T^{I J} .
$$

We observe that although $\mathrm{H} 1 \mathrm{c} \overline{2} \mathrm{~d} \overline{1}, \mathrm{H} 1 \mathrm{c} \overline{2} \mathrm{~d} 1, \mathrm{H} 1 \mathrm{c} 2 \mathrm{~d} \overline{1}$, and $\mathrm{H} 1 \mathrm{c} 2 \mathrm{~d} \overline{2}$ have more degrees of freedom than $\mathrm{H} 1 \mathrm{c} 1 \mathrm{~d} \overline{1}$, they do not result in more accurate approximations.

For studying convergence orders of the above CSFEMs, in Figure 10, we have plotted the $L^{2}$ norms of errors versus the maximum diameter $h$ for some uniform meshes. The convergence order of displacement is close to 2 for all methods. This is the optimal convergence order for all these CSFEMs except for $\mathrm{H} 2 \mathrm{c} 2 \mathrm{~d} \overline{2}$. The convergence order of $\boldsymbol{P}$ is close to 1 for all methods. The order of convergence of $\boldsymbol{K}$ is close to 1 for all methods except $\mathrm{H} 2 \mathrm{c} 2 \mathrm{~d} \overline{2}$, for which the convergence order is 2 . By considering some other meshes, we see that errors and convergence orders of $\boldsymbol{U}$ and $\boldsymbol{K}$ are not very sensitive to the underlying meshes. However, except for $\mathrm{H} 1 \mathrm{c} 2 \mathrm{~d} 1, \mathrm{H} 1 \mathrm{c} 2 \mathrm{~d} \overline{2}$, and $\mathrm{H} 2 \mathrm{c} 2 \mathrm{~d} \overline{2}$, errors and convergence rates of $\boldsymbol{P}$ are more mesh-dependent. Based on the above discussions, one may use H1c1d $\overline{1}, \mathrm{H} 1 \mathrm{c} 2 \mathrm{~d} 1$, and $\mathrm{H} 2 \mathrm{c} 2 \mathrm{~d} \overline{2}$, in practice.

Figure 11 shows the deformed configuration, $\|\boldsymbol{K}\|$, and $\|\boldsymbol{P}\|$ corresponding to the exact solution, $\mathrm{H} 1 \mathrm{c} 1 \mathrm{~d} \overline{1}$, and $\mathrm{H} 2 \mathrm{c} 2 \mathrm{~d} \overline{2}$. Solutions for CSFEMs are calculated for the mesh with 114 elements shown in Figure 9. Note that due to the interelement continuities of $H^{\mathbf{c}}$ and $H^{\mathbf{d}}$-conformal finite element spaces, $\|\boldsymbol{K}\|$ and $\|\boldsymbol{P}\|$ obtained using CSFEMs are not continuous, in general.


Figure 9: The shearing plate example: Geometry and boundary conditions (left), four unstructured meshes (right). 

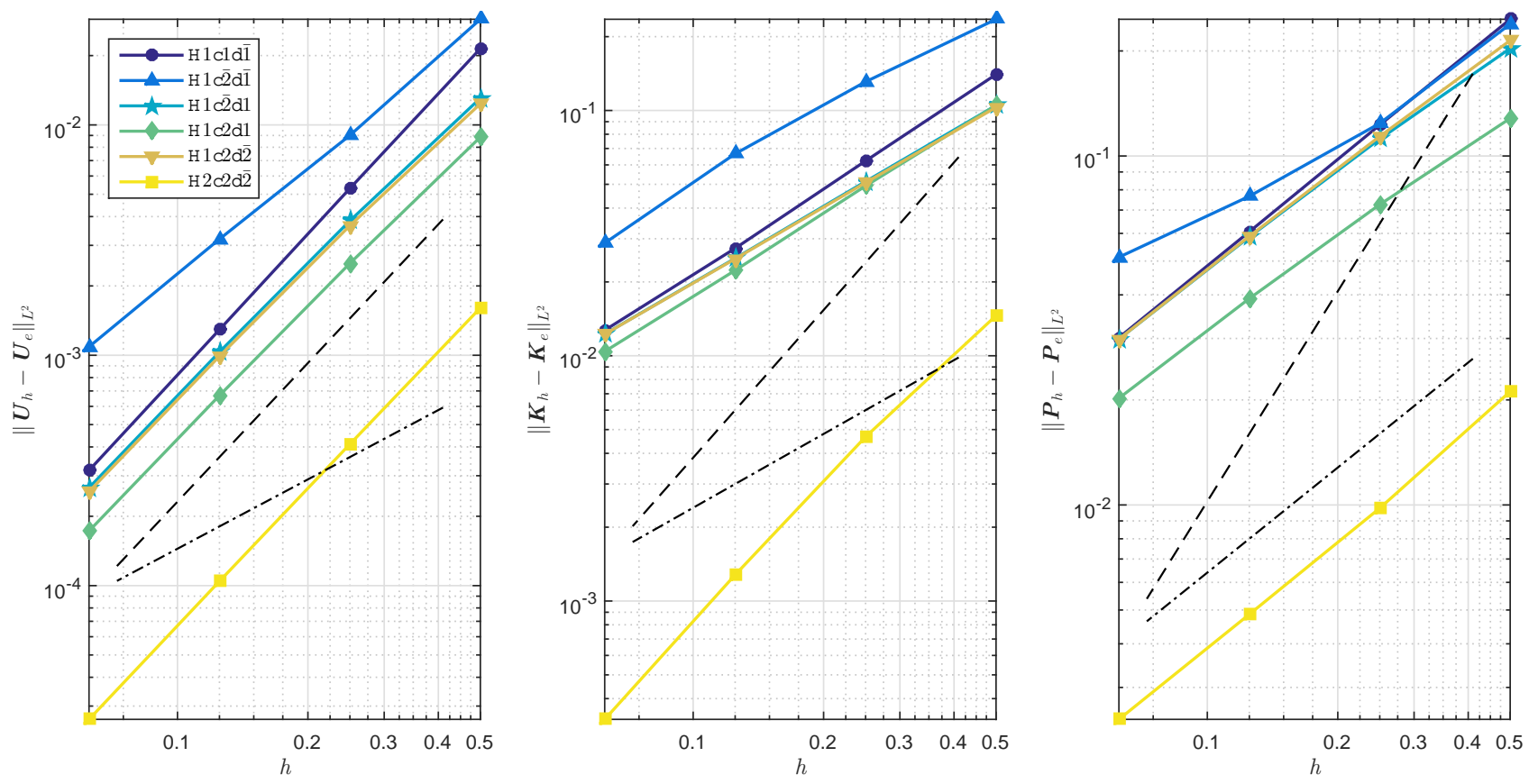

Figure 10: $L^{2}$-norms of errors in approximating displacement, displacement gradient, and stress versus the maximum diameter $h$ for the shearing plate example. In each diagram, different curves correspond to different CSFEMs. The points on each curve correspond to uniform meshes consisting of 16 ( $h=0.5), 64(h=0.25)$, 256 $(h=0.125)$, and 1024 $(h=0.0625)$ cells. In each diagram, the dash-dot line and the dashed line have the slopes 1 and 2 , respectively. 
Table 3: Convergence and error of different CSFEMs for the shearing plate example. DOF is the total number of degrees of freedom for each mesh, $\left(\boldsymbol{U}_{e}, \boldsymbol{F}_{e}, \boldsymbol{P}_{e}\right)$ is the exact solution, and $\left(\boldsymbol{U}_{h}, \boldsymbol{F}_{h}, \boldsymbol{P}_{h}\right)$ is the approximate solution for each CSFEM.

\begin{tabular}{|c|c|c|c|c|c|}
\hline CSFEM & \#Elements & \#DOF & $\left\|\boldsymbol{U}_{h}-\boldsymbol{U}_{e}\right\|_{L^{2}}$ & $\left\|\boldsymbol{K}_{h}-\boldsymbol{K}_{e}\right\|_{L^{2}}$ & $\left\|\boldsymbol{P}_{h}-\boldsymbol{P}_{e}\right\|_{L^{2}}$ \\
\hline \multirow{4}{*}{$\mathrm{H} 1 \mathrm{c} 1 \mathrm{~d} \overline{1}$} & 16 & 194 & $1.43 \mathrm{e}-02$ & $1.00 \mathrm{e}-01$ & $3.09 \mathrm{e}-01$ \\
\hline & 48 & 546 & $2.34 \mathrm{e}-03$ & $3.41 \mathrm{e}-02$ & $1.08 \mathrm{e}-01$ \\
\hline & 114 & 1238 & 8.92e-04 & $1.90 \mathrm{e}-02$ & $5.49 \mathrm{e}-02$ \\
\hline & 244 & 2586 & $3.42 \mathrm{e}-04$ & $8.94 \mathrm{e}-03$ & $3.70 \mathrm{e}-02$ \\
\hline \multirow{4}{*}{$\mathrm{H} 1 \mathrm{c} \overline{2} \mathrm{~d} \overline{1}$} & 16 & 258 & $2.46 \mathrm{e}-02$ & $2.01 \mathrm{e}-01$ & $3.01 \mathrm{e}-01$ \\
\hline & 48 & 738 & $4.93 \mathrm{e}-03$ & $7.82 \mathrm{e}-02$ & $1.13 \mathrm{e}-01$ \\
\hline & 114 & 1694 & $2.00 \mathrm{e}-03$ & $4.61 \mathrm{e}-02$ & $5.90 \mathrm{e}-02$ \\
\hline & 244 & 3562 & 7.31e-04 & $2.52 \mathrm{e}-02$ & $3.88 \mathrm{e}-02$ \\
\hline \multirow{4}{*}{$\mathrm{H} 1 \mathrm{c} \overline{2} \mathrm{~d} 1$} & 16 & 314 & $8.56 \mathrm{e}-03$ & $8.53 \mathrm{e}-02$ & $2.91 \mathrm{e}-01$ \\
\hline & 48 & 898 & $1.75 \mathrm{e}-03$ & $1.97 \mathrm{e}-02$ & $8.58 \mathrm{e}-02$ \\
\hline & 114 & 2060 & $6.95 \mathrm{e}-04$ & $9.66 \mathrm{e}-03$ & $2.94 \mathrm{e}-02$ \\
\hline & 244 & 4330 & $3.13 \mathrm{e}-04$ & $6.16 \mathrm{e}-03$ & $1.97 \mathrm{e}-02$ \\
\hline \multirow{4}{*}{$\mathrm{H} 1 \mathrm{c} 2 \mathrm{~d} \overline{1}$} & 16 & 346 & $9.53 \mathrm{e}-02$ & $5.77 \mathrm{e}-01$ & $1.89 \mathrm{e}-01$ \\
\hline & 48 & 994 & $2.39 \mathrm{e}-02$ & $3.69 \mathrm{e}-01$ & $9.03 \mathrm{e}-02$ \\
\hline & 114 & 2288 & $1.10 \mathrm{e}-02$ & $2.66 \mathrm{e}-01$ & $5.88 \mathrm{e}-02$ \\
\hline & 244 & 4818 & $5.57 \mathrm{e}-03$ & $2.01 \mathrm{e}-01$ & $3.87 \mathrm{e}-02$ \\
\hline \multirow{4}{*}{$\mathrm{H} 1 \mathrm{c} 2 \mathrm{~d} 1$} & 16 & 402 & $5.65 \mathrm{e}-03$ & $1.00 \mathrm{e}-01$ & $6.34 \mathrm{e}-02$ \\
\hline & 48 & 1154 & $1.44 \mathrm{e}-03$ & $2.65 \mathrm{e}-02$ & $2.09 \mathrm{e}-02$ \\
\hline & 114 & 2654 & $6.49 \mathrm{e}-04$ & $1.74 \mathrm{e}-02$ & $1.54 \mathrm{e}-02$ \\
\hline & 244 & 5586 & $2.93 \mathrm{e}-04$ & $1.01 \mathrm{e}-02$ & $8.72 \mathrm{e}-03$ \\
\hline \multirow{4}{*}{$\mathrm{H} 1 \mathrm{c} 2 \mathrm{~d} \overline{2}$} & 16 & 466 & $9.35 \mathrm{e}-03$ & $8.88 \mathrm{e}-02$ & $2.02 \mathrm{e}-01$ \\
\hline & 48 & 1346 & $3.14 \mathrm{e}-03$ & $4.59 \mathrm{e}-02$ & $1.18 \mathrm{e}-01$ \\
\hline & 114 & 3110 & $1.32 \mathrm{e}-03$ & $2.99 \mathrm{e}-02$ & $8.17 \mathrm{e}-02$ \\
\hline & 244 & 6562 & $6.37 \mathrm{e}-04$ & $1.97 \mathrm{e}-02$ & $5.38 \mathrm{e}-02$ \\
\hline \multirow{4}{*}{$\mathrm{H} 2 \mathrm{c} 2 \mathrm{~d} \overline{2}$} & 16 & 522 & $1.50 \mathrm{e}-03$ & $1.51 \mathrm{e}-02$ & $2.66 \mathrm{e}-02$ \\
\hline & 48 & 1506 & $4.14 \mathrm{e}-04$ & $4.65 \mathrm{e}-03$ & $1.04 \mathrm{e}-02$ \\
\hline & 114 & 3476 & $1.80 \mathrm{e}-04$ & $2.13 \mathrm{e}-03$ & $6.47 \mathrm{e}-03$ \\
\hline & 244 & 7330 & $8.07 \mathrm{e}-05$ & $9.67 \mathrm{e}-04$ & $4.17 \mathrm{e}-03$ \\
\hline
\end{tabular}



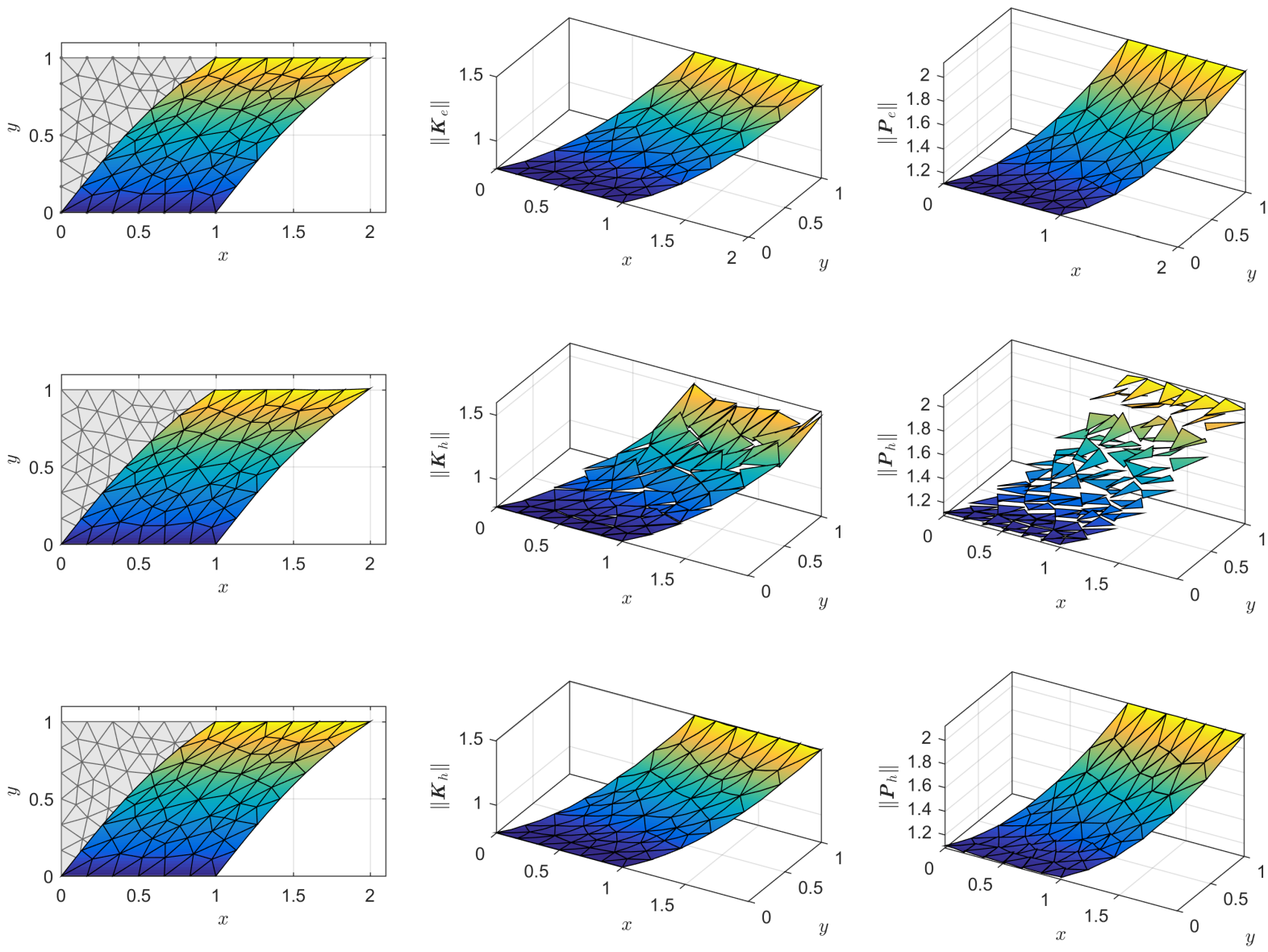

Figure 11: The comparison between the deformed configuration, the norm of displacement gradient $\|\boldsymbol{K}\|$, and the norm of stress $\|\boldsymbol{P}\|$ associated to the exact solution of the shearing plate example and those obtained by CSFEMs. Colors indicate values of $\|\boldsymbol{U}\|,\|\boldsymbol{K}\|$, and $\|\boldsymbol{P}\|$ in the first, the second, and the third columns, respectively. Lighter colors indicate larger values. The first, the second, and the third rows correspond to the exact solution, $\mathrm{H} 1 \mathrm{c} 1 \mathrm{~d} \overline{1}$, and $\mathrm{H} 2 \mathrm{c} 2 \mathrm{~d} \overline{2}$, respectively. The underlying mesh of CSFEMs has 114 cells. 


\subsection{Example 2: Cook's Membrane}

For studying the performance of CSFEMs in bending and in the near-incompressible regime, we consider the standard Cook's membrane problem shown in Figure 12. We use the energy function $W_{2}(\boldsymbol{F})$, with $\mu=80.194 \mathrm{~N} / \mathrm{mm}^{2}$, and $\kappa=400889.8 \mathrm{~N} / \mathrm{mm}^{2}$.


Figure 12: Cook's membrane: Geometry, boundary conditions, and three unstructured meshes.
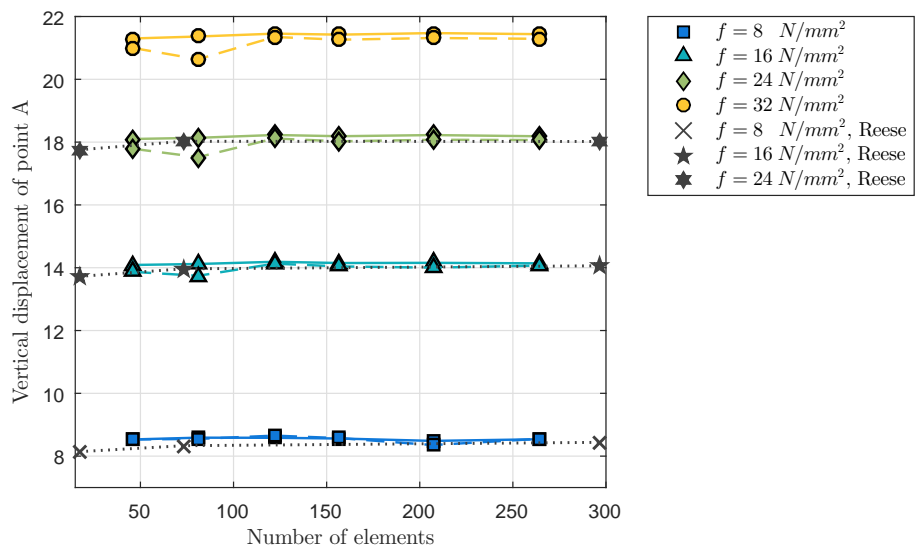

Figure 13: Cook's membrane: Vertical displacement of point A in Figure 12 for different values of the shearing force $f$ versus the number of elements in the mesh. The dashed and the solid lines are the results obtained by $\mathrm{H} 1 \mathrm{c} 1 \mathrm{~d} \overline{1}$ and $\mathrm{H} 2 \mathrm{c} 2 \mathrm{~d} \overline{2}$, respectively. The underlying meshes have 46, 81, 122, 15\%, 207, and 264 elements. The dotted lines indicate the results of Reese [46]. 

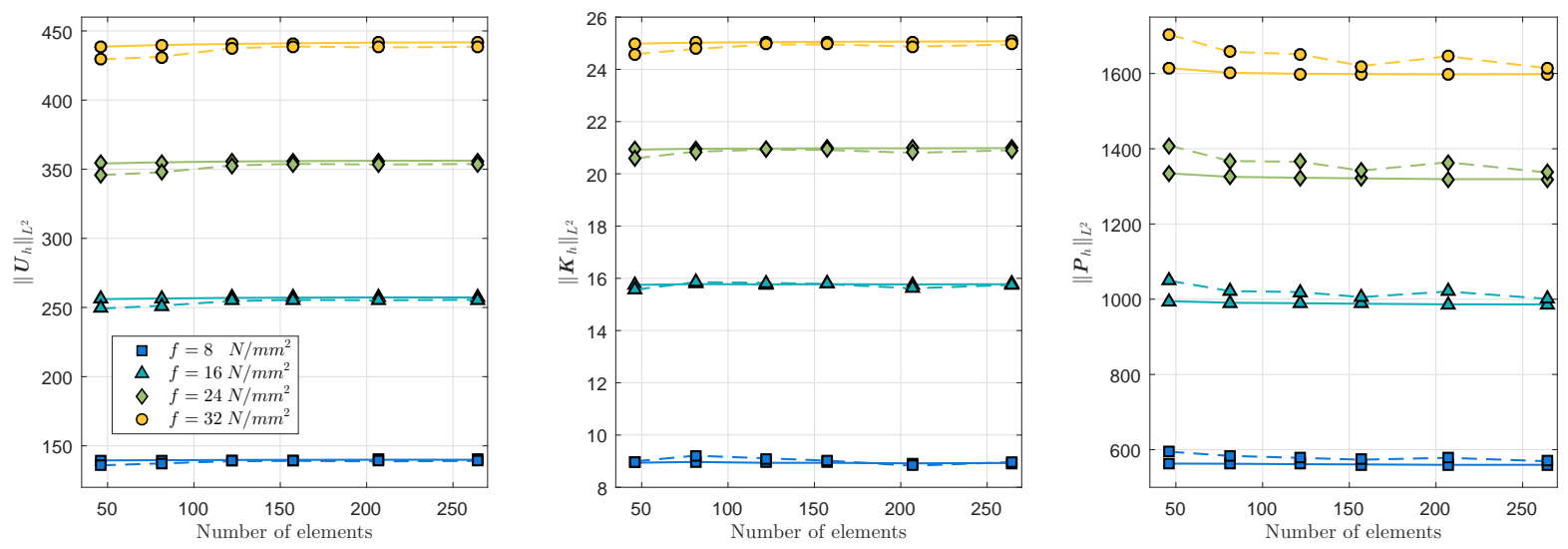

Figure 14: Cook's membrane: $L^{2}$-norms of displacement, displacement gradient, and stress (for different values of the shearing force $f$ ) versus the number of elements in the mesh. The dashed and the solid lines are obtained by $\mathrm{H} 1 \mathrm{cld} \mathbf{1}$ and $\mathrm{H} 2 \mathrm{c} 2 \mathrm{~d} \overline{2}$, respectively. The underlying meshes have 46, 81, 122, 15\%, 207, and 264 elements.
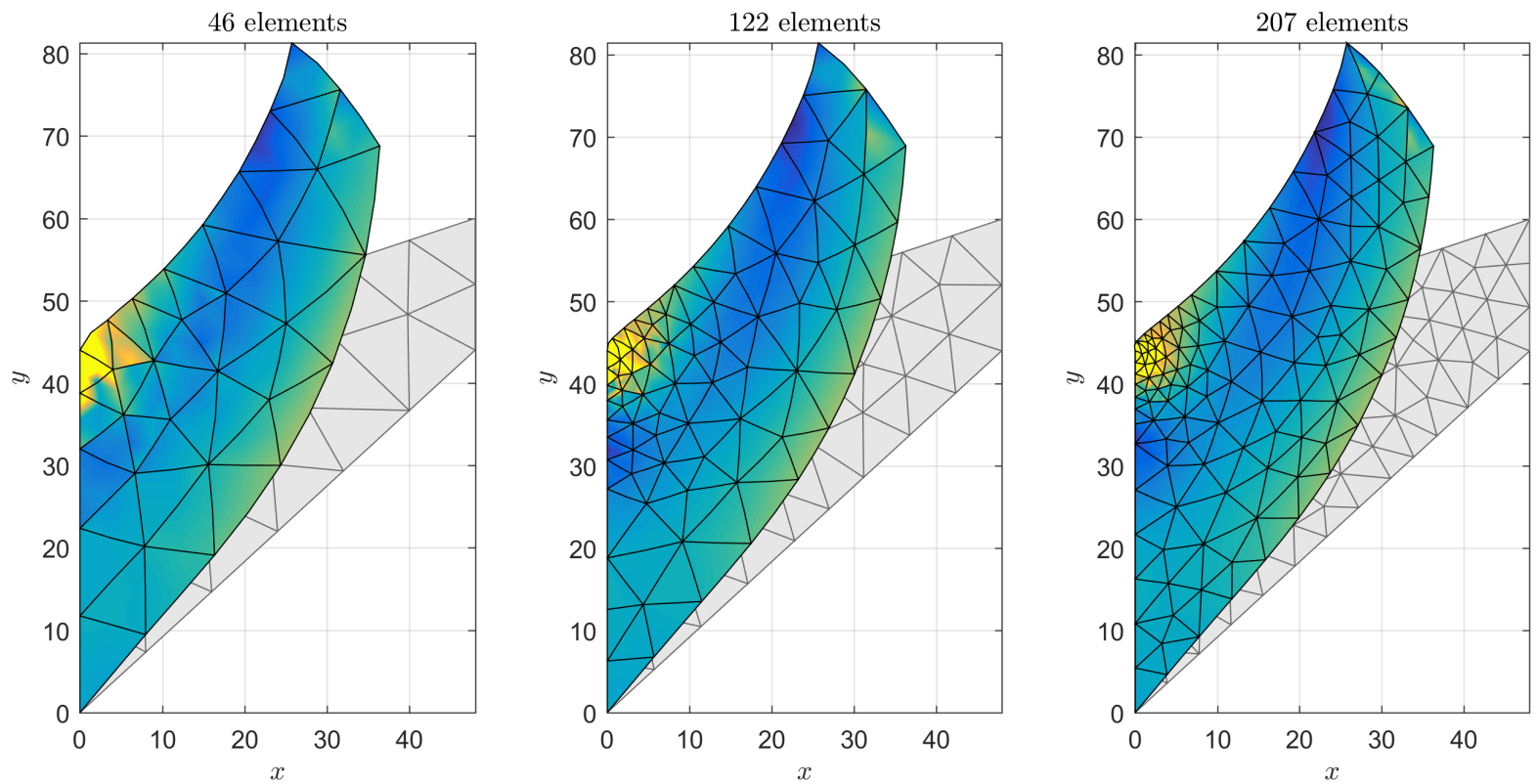

Figure 15: Deformed configurations of Cook's membrane using $\mathrm{H} 2 \mathrm{c} 2 \mathrm{~d} \overline{2}$ and the meshes of Figure 12, with the shear force $f=32 \mathrm{~N} / \mathrm{mm}^{2}$. Colors indicate values of the norm of stress $\|\boldsymbol{P}\|$, where lighter colors correspond to larger values of $\|\boldsymbol{P}\|$. 
To investigate the pointwise convergence of the approximate solutions, in Figure 13, we have plotted vertical displacement of point $A$ for different meshes and for different values of the shearing force $f$. We use H1c1d $\overline{1}$ and $\mathrm{H} 2 \mathrm{c} 2 \mathrm{~d} \overline{2}$ and compare our results with those of Reese [46]. We observe that both of these CSFEMs give good approximations for relatively coarse meshes. Figure 14 shows the global convergence of $\mathrm{H} 1 \mathrm{c} 1 \mathrm{~d} \overline{1}$ and $\mathrm{H} 2 \mathrm{c} 2 \mathrm{~d} \overline{2}$. We plot $L^{2}$-norms of displacement, displacement gradient, and stress for different meshes. One observes that these CSFEMs have comparable accuracy for $\boldsymbol{U}$ and $\boldsymbol{K}$. However, as the shearing force increases, H1c1d $\overline{1}$ becomes less accurate in approximating $\boldsymbol{P}$. Figure 15 shows the deformed configuration of Cook's membrane for the meshes of Figure 12 by using $\mathrm{H} 2 \mathrm{c} 2 \mathrm{~d} \overline{2}$ and $f=32 \mathrm{~N} / \mathrm{mm}^{2}$. Colors in this figure indicate the values of $\|\boldsymbol{P}\|:=\left(\sum_{I, J} P^{I J} P^{I J}\right)^{1 / 2}$, with lighter colors corresponding to larger values of $\|\boldsymbol{P}\|$.

\subsection{Example 3: Inhomogeneous Compression}

Next, we consider a plate under compression shown in Figure 16. The horizontal (vertical) displacement of the top (bottom) of the plate is constrained to be zero, and the material properties are the same as those of the previous example. Many enhanced strain methods suffer from the hourglass instability in this problem, e.g. see Reese [46] and references therein.

Due to the symmetry of this problem, as shown in Figure 16, we only consider the right half of the plate. Figure 17 depicts the vertical displacement of point $A$ in Figure 16 for different values of the force $f$. Displacements are obtained by using $\mathrm{H} 2 \mathrm{c} 2 \mathrm{~d} \overline{2}$. Our results agree with those of Reese [46], which are obtained by using hourglass stabilization techniques. We observe that all the first-order and second-order CSFEMs except $\mathrm{H} 2 \mathrm{c} 2 \mathrm{~d} \overline{2}$ become unstable if the displacement of point $A$ is more than $30 \%$ of the height of the plate. However, $\mathrm{H} 2 \mathrm{c} 2 \mathrm{~d} \overline{2}$ remains stable without using any additional stabilization technique. Figure 18 shows $L^{2}$-norms of $\boldsymbol{U}, \boldsymbol{K}$, and $\boldsymbol{P}$. One observes that $\mathrm{H} 2 \mathrm{c} 2 \mathrm{~d} \overline{2}$ has a good global convergence behavior in this example. Finally, in Figure 19, we have plotted the deformed configuration and $\|\boldsymbol{P}\|$ for the force $f=600 \mathrm{~N} / \mathrm{mm}^{2}$, which results in displacement of point A being $65 \%$ of plate height.
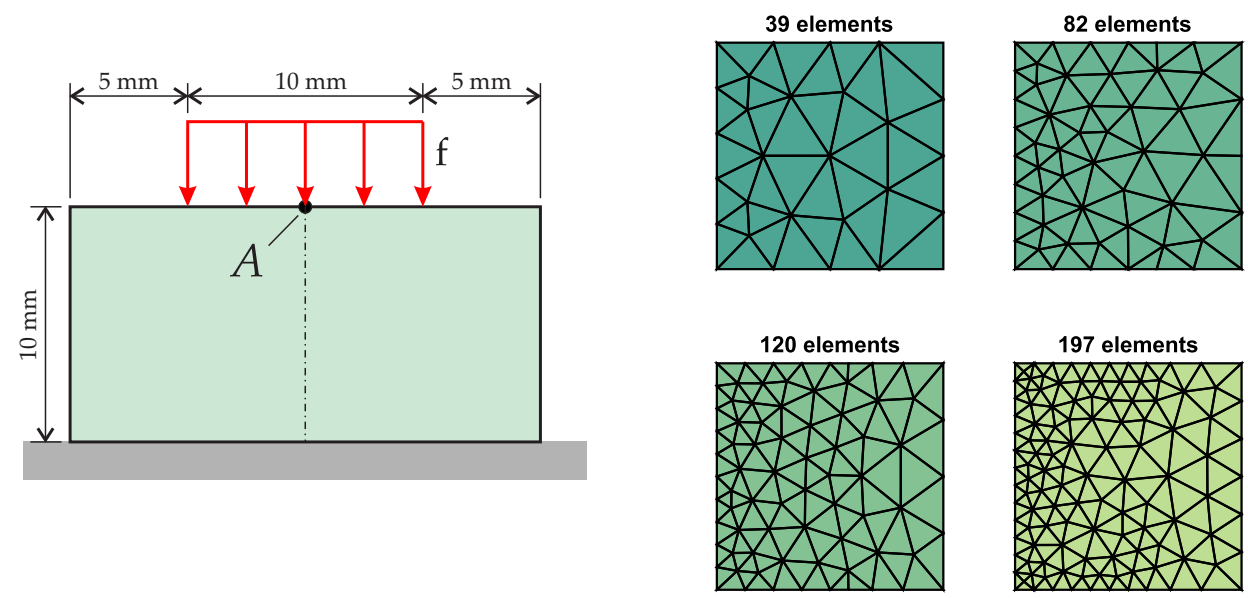

Figure 16: The inhomogeneous compression example: Geometry, boundary conditions, and four unstructured meshes. Note that using the symmetry of this example, only half of the plate is modeled. 




Figure 17: The inhomogeneous compression example: Vertical displacement of point A of Figure 16 for different values of the force $f$ versus the number of elements in the mesh. The results are obtained by using $\mathrm{H} 2 \mathrm{c} 2 \mathrm{~d} \overline{2}$. The underlying meshes have 39, 82, 120, 160, 197, and 236 elements. The dotted lines indicate the results of Reese [46].
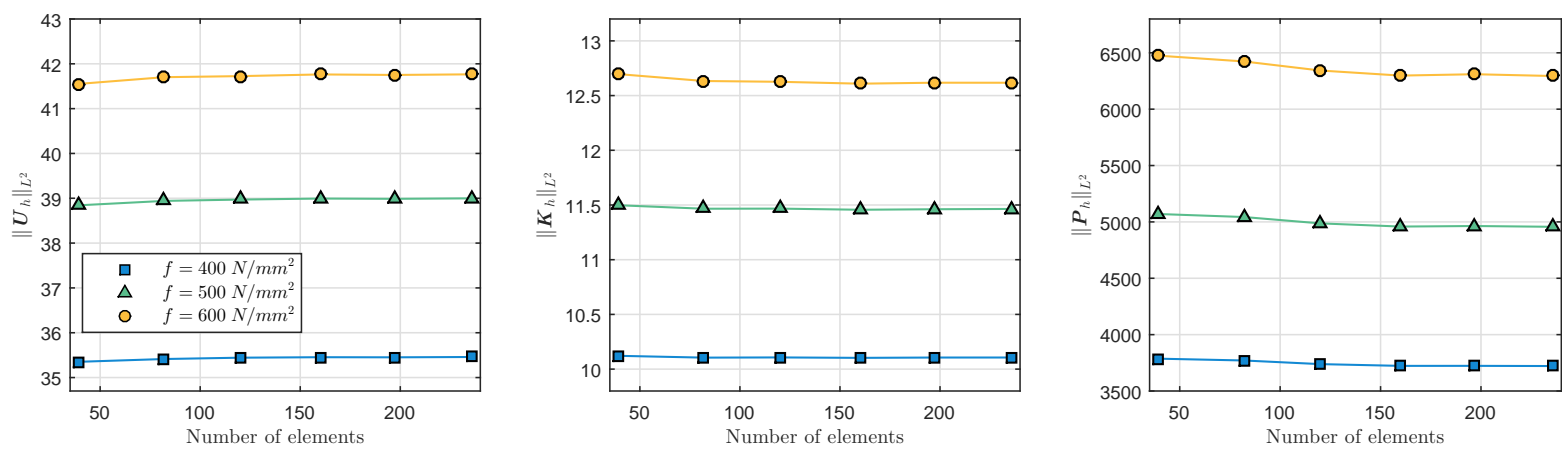

Figure 18: The inhomogeneous compression example: $L^{2}$-norms of displacement, displacement gradient, and stress (for different values of the compressing force $f$ ) versus the number of elements in the mesh. The results are obtained by using $\mathrm{H} 2 \mathrm{c} 2 \mathrm{~d} \overline{2}$. The underlying meshes have 39, 82, 120, 160, 197, and 236 elements.

\subsection{Example 4: Rubber Sealing}

Next, we consider the compression of a rubber sealing studied in [47]. The geometry of the sealing is shown in Figure 20. The Ogden material with energy function $W_{3}(\boldsymbol{F})$ given in $(3.45 \mathrm{c})$ is assumed with the following parameters

$$
\begin{aligned}
\kappa & =1000 \mathrm{~N} / \mathrm{mm}^{2}, m=3 \\
\mu_{1} & =0.63 \mathrm{~N} / \mathrm{mm}^{2}, \mu_{2}=0.0012 \mathrm{~N} / \mathrm{mm}^{2}, \mu_{3}=-0.01 \mathrm{~N} / \mathrm{mm}^{2}, \\
\alpha_{1} & =1.3, \alpha_{2}=5, \alpha_{3}=-2 .
\end{aligned}
$$

We impose a vertical displacement $v$ on the top of the sealing. Due to the symmetry of the problem, we only consider half of the sealing. Four unstructred meshes of the sealing are shown in Figure 20.

In Figure 21, we have plotted $L^{2}$-norms of $\boldsymbol{U}_{h}, \boldsymbol{K}_{h}$, and $\boldsymbol{P}_{h}$ for different meshes under various vertical displacements $v$. The dashed and the solid curves are calculated by using $\mathrm{H} 1 \mathrm{c} 1 \mathrm{~d} \overline{1}$ and $\mathrm{H} 2 \mathrm{c} 2 \mathrm{~d} \overline{2}$, respectively. We observe that $\mathrm{H} 2 \mathrm{c} 2 \mathrm{~d} \overline{2}$ converges rapidly. However, $\mathrm{H} 1 \mathrm{c} 1 \mathrm{~d} \overline{1}$ has a poor performance in approximating $\boldsymbol{K}$ and $\boldsymbol{P}$. Figure 22 shows the deformed configurations of the sealing with $v=$ $-2.2 \mathrm{~mm}$ using the meshes of Figure 20. The results are obtained using $\mathrm{H} 2 \mathrm{c} 2 \mathrm{~d} \overline{2}$ and colors indicate 

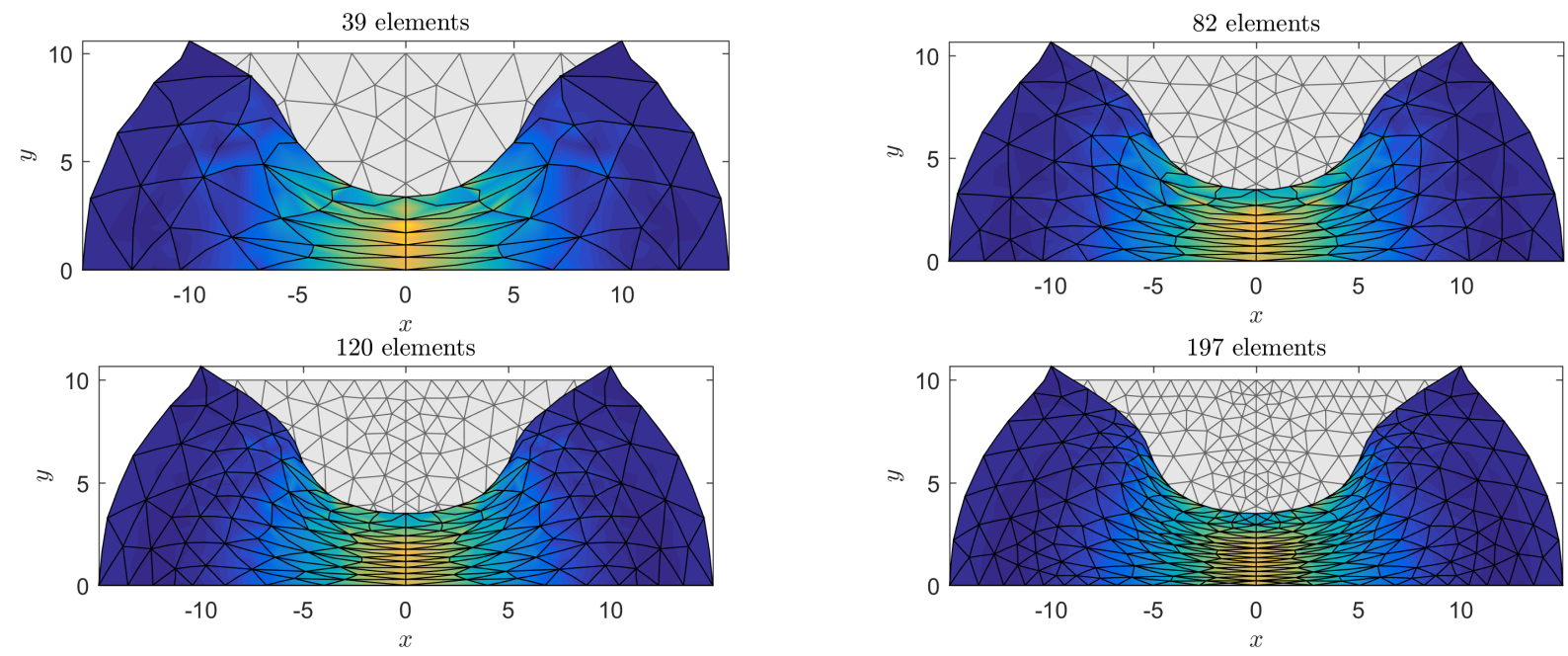

Figure 19: Deformed configurations of the inhomogeneous compression example using $\mathrm{H} 2 \mathrm{c} 2 \mathrm{~d} \overline{2}$ and the meshes of Figure 16. The plate is under the force $f=600 \mathrm{~N} / \mathrm{mm}^{2}$, which results in displacement at the middle point A being $65 \%$ of the plate height. Colors indicate values of the norm of stress $\|\boldsymbol{P}\|$ with lighter colors corresponding to higher values of $\|\boldsymbol{P}\|$.

values of $\|\boldsymbol{P}\|$, where lighter colors correspond to higher values.

\subsection{Example 5: A Plate with Randomly Distributed Holes}

For studying the performance of CSFEMs on domains with complex geometries, we consider a square plate containing some randomly distributed holes with arbitrary shapes as shown in Figure 23. The material properties are the same as those of the Cook's membrane example. We fix the bottom of the plate and impose the displacement field $\boldsymbol{U}=(0, v)$ at the top of the plate. Figure 23 shows three unstructured meshes for the plate. In Figure 24, we have plotted the $L^{2}$-norms of $\boldsymbol{U}, \boldsymbol{K}$, and $\boldsymbol{P}$ for various meshes under different values of the vertical displacement $v$ at the top of the plate. The results are calculated using $\mathrm{H} 2 \mathrm{c} 2 \mathrm{~d} \overline{2}$. One observes that even relatively coarse meshes lead to good approximations for stress. Figure 25 depicts the deformed configurations and the norm $\|\boldsymbol{P}\|$ associated to the meshes of Figure 23. The vertical displacement $v=0.5 \mathrm{~mm}$ is imposed at the top of the plate.

\subsection{Example 6: Tension of a Heterogeneous Plate}

As was mentioned in $§ 3.1 .2$, the underlying $H^{\mathbf{c}}$ and $H^{\mathbf{d}}$-conformal finite element spaces of CSFEMs automatically satisfy the Hadamard jump condition and the zero traction jumps at the internal edges, respectively. This fact can be used to model certain types of heterogeneous materials. More specifically, if inhomogeneities do not slide at the interfaces, i.e. at internal interfaces displacement is continuous and traction vectors are single-valued, then one can consider a single mesh for the entire heterogeneous structure and only associate different material properties to different elements depending on their locations.

To demonstrate the idea, we consider a simple heterogeneous square plate shown in Figure 26. The plate size is $1 \mathrm{~mm} \times 1 \mathrm{~mm}$ and there is a circular inhomogeneity of radius $0.25 \mathrm{~mm}$ at the center of the plate. The bottom of the plate is fixed and a $1 \mathrm{~mm}$ vertical displacement (100\% stretch) is imposed on the top. Figure 26 shows four unstructured meshes for the plate. Instead of considering two separate meshes for the plate and the inhomogeneity and imposing suitable boundary conditions 

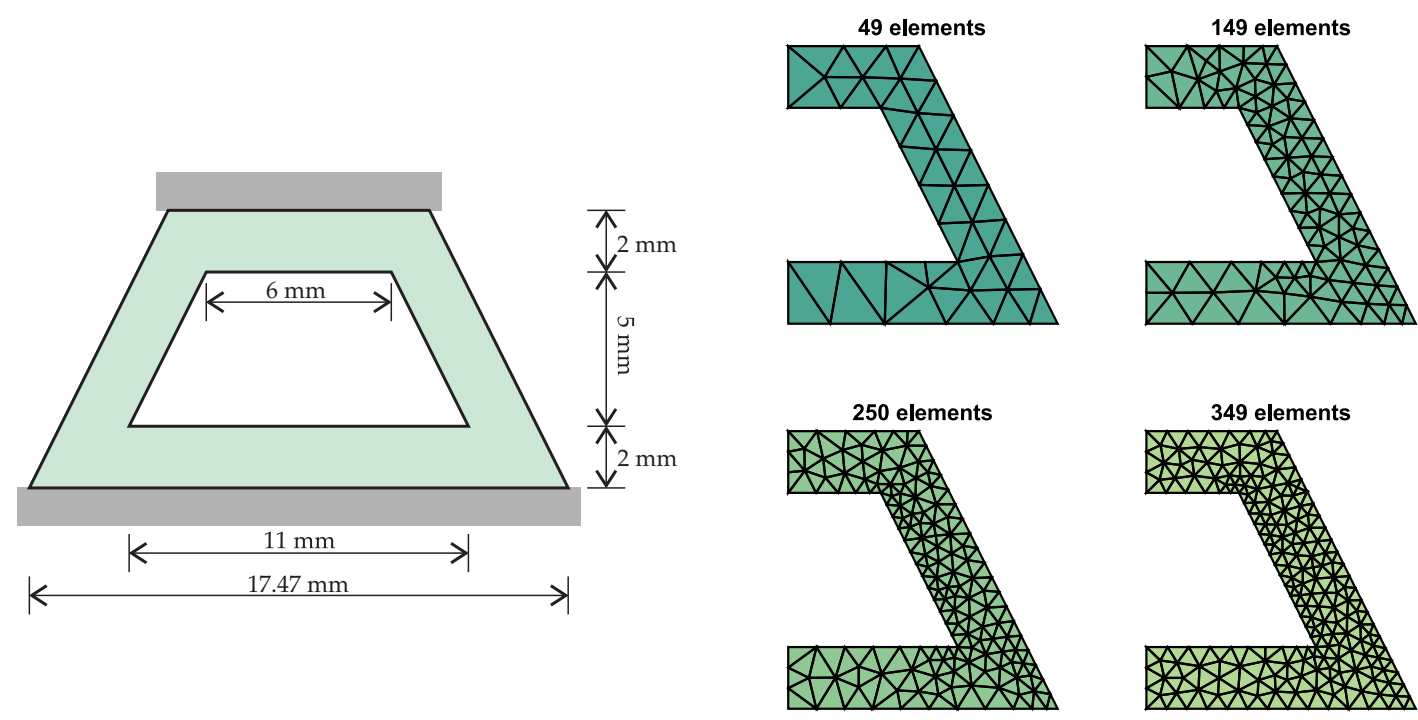

Figure 20: The rubber sealing example: Geometry, boundary conditions, and four unstructured meshes. Using symmetry of this problem, only half of the sealing is modeled.

on their interfaces, we use a single mesh for the $1 \mathrm{~mm} \times 1 \mathrm{~mm}$ square and associate different material properties to elements depending on their position.

The energy function $W_{2}(\boldsymbol{F})$ with $(\kappa, \mu)=(120.291,80.194) \mathrm{N} / \mathrm{mm}^{2}$ is assumed for the plate. For the inhomogeneity, we use $W_{2}(\boldsymbol{F})$ with $(\kappa, \mu)=(\bar{\kappa}, \bar{\mu})$. The following four cases are studied: (i) A homogeneous plate: $(\bar{\kappa}, \bar{\mu})=(120.291,80.194) \mathrm{N} / \mathrm{mm}^{2}$, (ii) a plate with a hole: $(\bar{\kappa}, \bar{\mu})=$ $\left(10^{-9}, 10^{-9}\right) \mathrm{N} / \mathrm{mm}^{2}$, (iii) $(\bar{\kappa}, \bar{\mu})=(481.164,320.776) \mathrm{N} / \mathrm{mm}^{2}$, (iv) a rigid inhomogeneity: $(\bar{\kappa}, \bar{\mu})=$ $\left(10^{9}, 10^{9}\right) \mathrm{N} / \mathrm{mm}^{2}$. Figure 27 shows the convergence of solutions for these cases. The dashed and solid curves are obtained by using $\mathrm{H} 1 \mathrm{c} 1 \mathrm{~d} \overline{1}$ and $\mathrm{H} 2 \mathrm{c} 2 \mathrm{~d} \overline{2}$, respectively. One observes that except for the rigid inhomogeneity case, H1c1d $\overline{1}$ has a good performance.

Figure 28 depicts the deformed configuration of the case (iii) for the meshes of Figure 26. The results are calculated by using $\mathrm{H} 2 \mathrm{c} 2 \mathrm{~d} \overline{2}$ and colors indicate the values of the stress norm $\|\boldsymbol{P}\|$, where lighter colors correspond to higher values of $\|\boldsymbol{P}\|$. Finally, we have plotted the deformed configurations of the above four cases in Figure 29. The results are calculated by using $\mathrm{H} 2 \mathrm{c} 2 \mathrm{~d} \overline{2}$ and the underlying mesh has 200 elements. Colors indicate $\|\boldsymbol{P}\|$ and are normalized between all the deformed configurations so that the same colors in different configurations indicate the same values of $\|\boldsymbol{P}\|$. As one expects, $\|\boldsymbol{P}\|$ increases as the inhomogeneity becomes stiffer. For comparison purposes, we also considered tension of the same plate with a circular hole instead of the inhomogeneity and observed that the results coincide with those of case (ii) with a very soft inhomogeneity.

\section{Concluding Remarks}

In this paper, we introduced a new class of mixed finite element methods for nonlinear elasticity compatible-strain mixed finite element methods (CSFEMs). These finite element methods are based on a Hu-Washizu type functional with the displacement $\boldsymbol{U}$, the displacement gradient $\boldsymbol{K}$, and the first Piola-Kirchhoff stress tensor $\boldsymbol{P}$ as the independent unknowns. The trial spaces for $\boldsymbol{K}$ and $\boldsymbol{P}$ are assumed to be the Hilbert spaces $H^{\mathbf{c}}$ and $H^{\mathbf{d}}$, respectively. The differential operators $\mathbf{c}$ (the 2D curl operator) and $\mathbf{d i v}$ can be weakly defined on $H^{\mathbf{c}}$ and $H^{\mathbf{d}}$, respectively. The choice of the trial space $H^{\mathbf{c}}$ for $\boldsymbol{K}$ follows form the necessary compatibility condition for $\boldsymbol{K}$, i.e. $\mathbf{c}(\boldsymbol{K})=\mathbf{0}$. By using $H^{\mathbf{c}}$ and 

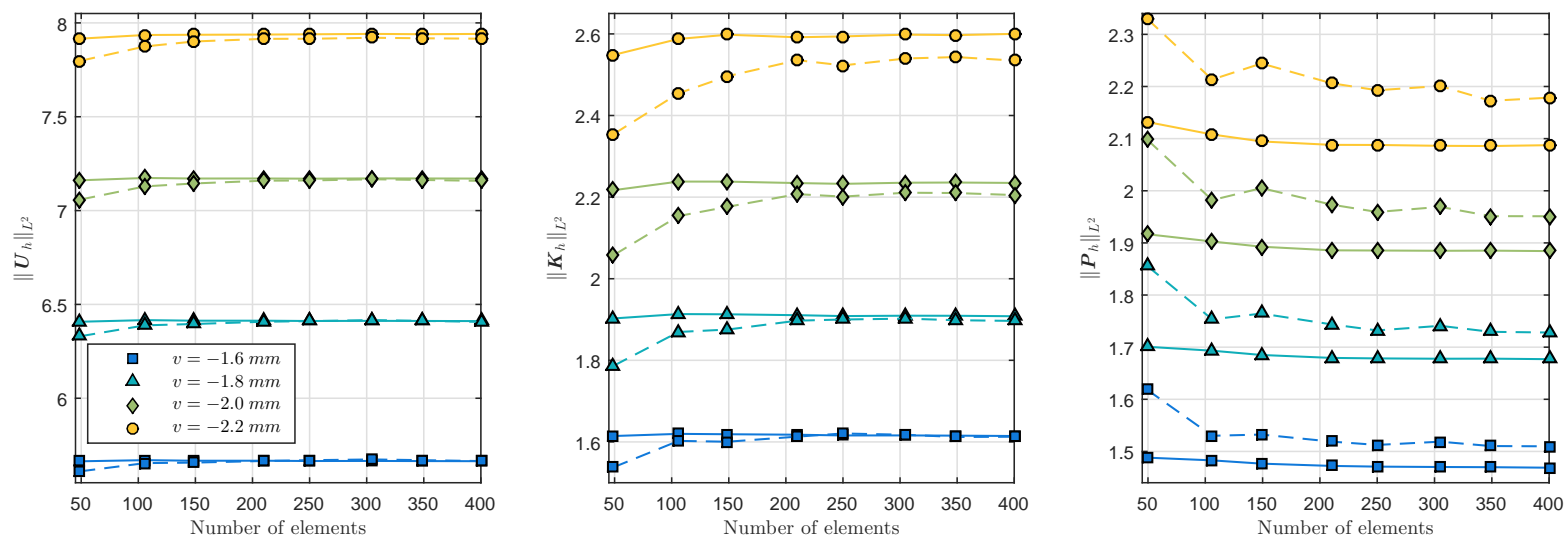

Figure 21: The rubber sealing example: $L^{2}$-norms of displacement, displacement gradient, and stress versus the number of elements in the mesh. Different curves indicate results associated to different values of the vertical displacement $v$ at the sealing upper boundary. The dashed and the solid curves are obtained using $\mathrm{H} 1 \mathrm{c} 1 \mathrm{~d} \overline{1}$ and $\mathrm{H} 2 \mathrm{c} 2 \mathrm{~d} \overline{2}$, respectively. The underlying meshes have 49, 106, 149, 210, 250, 305, 349, and 400 elements.

$H^{\mathrm{d}}$, one can write two Hilbert complexes for $2 \mathrm{D}$ nonlinear elasticity that decribe the kinematics and the kinetics of large deformations. By utilizing the relation between these Hilbert complexes and the celebrated de Rham complex, we discretized the Hilbert complexes using the finite element exterior calculus. CSFEMs are obtained by replacing solution spaces of the above mixed formulation with suitable finite element spaces of the discrete complexes.

In comparison with the standard finite element methods for nonlinear elasticity, CSFEMs require more degrees of freedom. However, we observed that CSFEMs have good performances for problems for which many standard finite element methods fail or have a poor performance. In particular, CSFEMs give accurate approximations of stress on domains with complex geometries. Such a performance is highly desirable in many applications such as fracture mechanics. Moreover, CSFEMs provide a convenient framework for modeling inhomogeneities. It is possible to extend CSFEMs to 3D nonlinear elasticity. To this end, one should use the 3D counterpart of the Hilbert complexes (2.1) and (2.2). In future communications, we will extend CSFEMs to 3D nonlinear elasticity and also to incompressible nonlinear elasticity.

Acknowledgments. MFS and AY were partially supported by AFOSR - Grant No. FA9550-10-10378 and FA9550-12-1-0290 and NSF - Grant No. CMMI 1042559 and CMMI 1130856.

\section{References}

[1] P. Wriggers and S. Reese. A note on enhanced strain methods for large deformations. Comput. Methods Appl. Mech. Engrg., 135:201-209, 1996.

[2] F. Auricchio, L. da Veiga Beirao, C. Lovadina, A. Reali, R. L. Taylor, and P. Wriggers. Approximation of incompressible large deformation elastic problems: Some unresolved issues. Comput. Mech., 52:1153-1167, 2013.

[3] F. Auricchio, L. da Veiga Beirao, C. Lovadina, and A. Reali. Stability of some finite element methods for finite elasticity problems. In Mixed finite element technologies, pages 179-206. CISM Courses and Lectures, Springer-Verlag, Wien, 2009. 

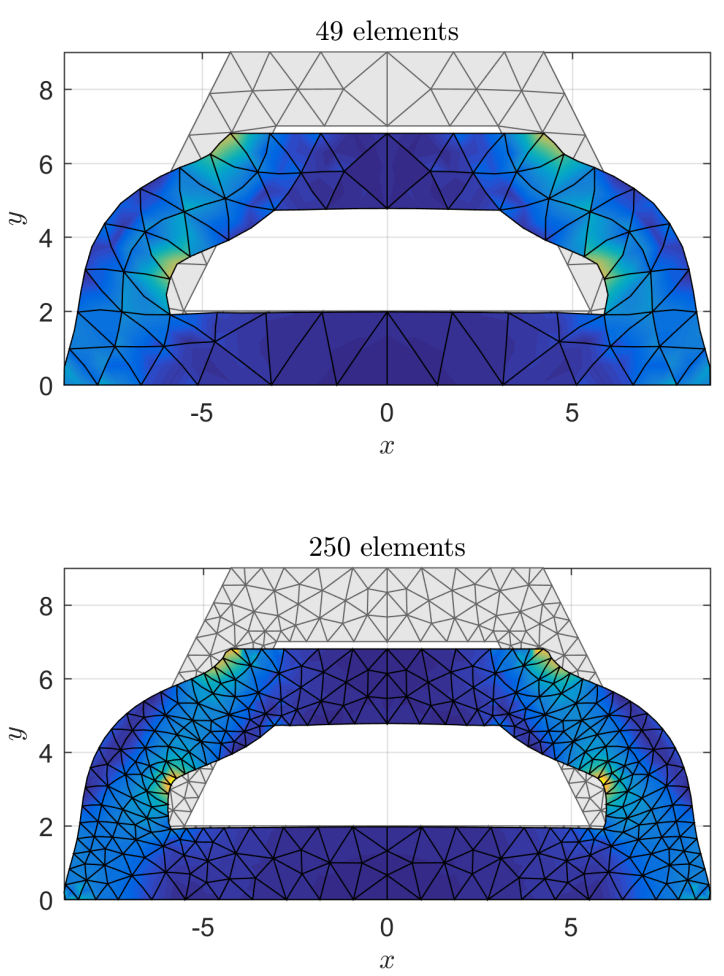
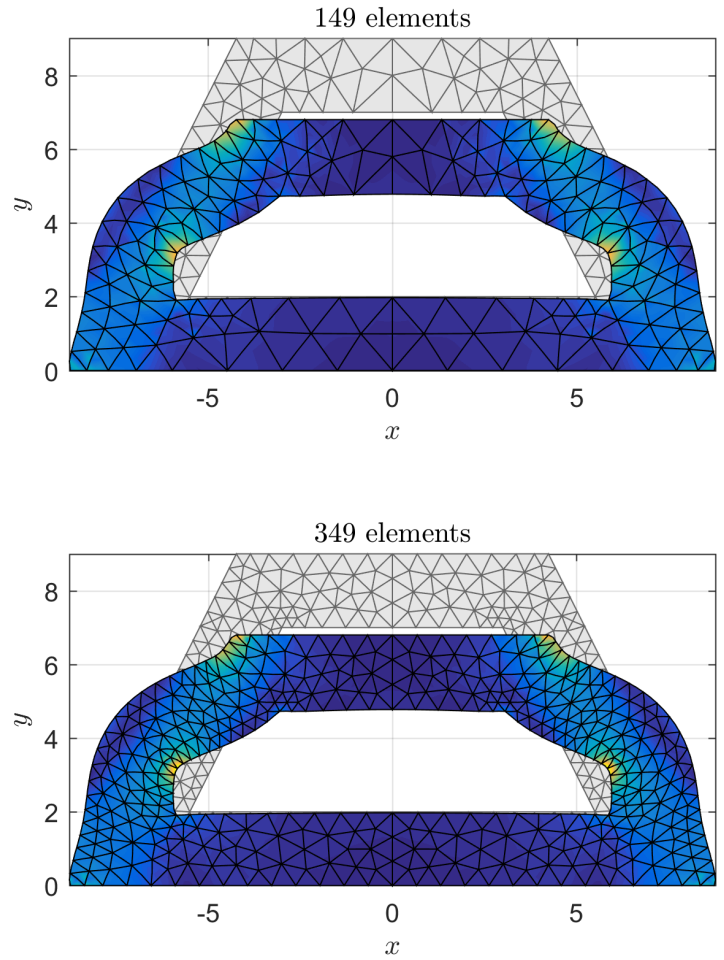

Figure 22: Deformed configurations of the rubber sealing example using $\mathrm{H} 2 \mathrm{c} 2 \mathrm{~d} \overline{2}$ and the meshes of Figure 20. The imposed vertical displacement at the upper boundary of the sealing is $v=-2.2 \mathrm{~mm}$ (almost $25 \%$ shortening). Colors indicate values of the norm of stress $\|\boldsymbol{P}\|$, where lighter colors correspond to higher values of $\|\boldsymbol{P}\|$.

[4] T. Belytschko, Y. Krongauz, D. Organ, M. Fleming, and P. Krysl. Meshless methods: An overview and recent developments. Comput. Methods in Appl. Mech. Eng., 139:3-47, 1996.

[5] T. Belytschko, YY. Lu, and L. Gu. Element-free Galerkin methods. Int. J. Numer. Meth. Eng., 37:229-256, 1994.

[6] GH. Shi. Manifold method of material analysis. Transactions of the 9th Army Conference On Applied Mathematics and Computing, Report, No.92-1, U.S. Army Research Office, 1991.

[7] G. MA, X. AN, and L. HE. The numerical manifold method: A review. Int. J. Comput. Methods, $7: 1-32,2010$.

[8] J. M. Melenk and I. Babuška. The partition of unity finite element method: basic theory and applications. Comput. Methods in Appl. Mech. Eng., 139:289-314, 1996.

[9] I. Babuška and J. M. Melenk. The partition of unity method. Int. J. Numer. Meth. Eng., 40: 727-758, 1997.

[10] T. Strouboulis, K. Copps, and I. Babuška. The generalized finite element method. Comput. Methods Appl. Mech. Engrg., 190:4081-4193, 2001.

[11] I. Babuška and U. Banerjee. Stable generalized finite element method (SGFEM). Comput. Methods Appl. Mech. Engrg., 201-204:91-111, 2012. 

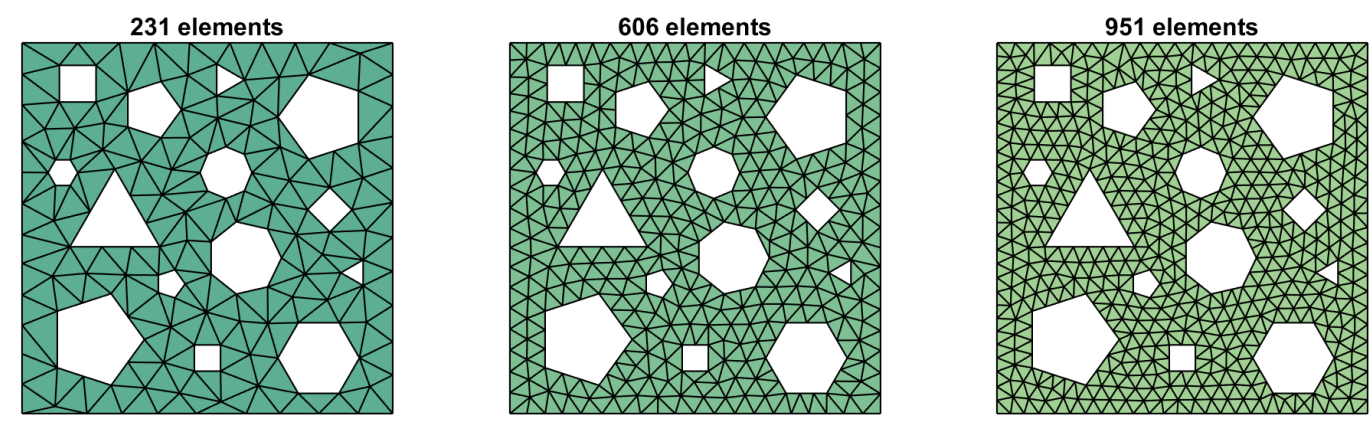

Figure 23: Three unstructured meshes for a plate with randomly distributed holes.


Figure 24: A plate with randomly distributed holes: $L^{2}$-norms of displacement, displacement gradient, and stress versus the number of elements in the mesh. Different curves indicate the results for different values of the vertical displacement $v$ at the upper boundary of the plate. The results are obtained by using $\mathrm{H} 2 \mathrm{c} 2 \mathrm{~d} \overline{2}$. The underlying meshes have 139, 231, 407, 606, 789, 951, and 1033 elements.

[12] K.R. Srinivasan, K. Matouš, and P.H. Geubelle. Generalized finite element method for modeling nearly incompressible bimaterial hyperelastic solids. Comput. Methods Appl. Mech. Engrg., 197: 4882-4893, 2008.

[13] T. P. Fries and T. Belytschko. The extended/generalized finite element method: An overview of the method and its applications. Int. J. Numer. Meth. Eng., 84:253-304, 2010.

[14] Y. Abdelaziz and A. Hamouine. A survey of the extended finite element. Comput. Struct., 86: 1141-1151, 2008.

[15] A. R. Khoei, S. O. R. Biabanaki, and M. Anahid. Extended finite element method for threedimensional large plasticity deformations. Comput. Methods Appl. Mech. Eng., 197:1100-1114, 2008.

[16] O. C. Zienkiewicz, R. L. Taylor, and J. M. Too. Reduced integration technique in general analysis of plates and shells. Int. J. Numer. Meth. Eng., 3:275-290, 1971.

[17] T. J. R. Hughes. Generalization of selective integration procedures to anisotropic and nonlinear media. Int. J. Numer. Meth. Eng., 15:1413-1418, 1980. 

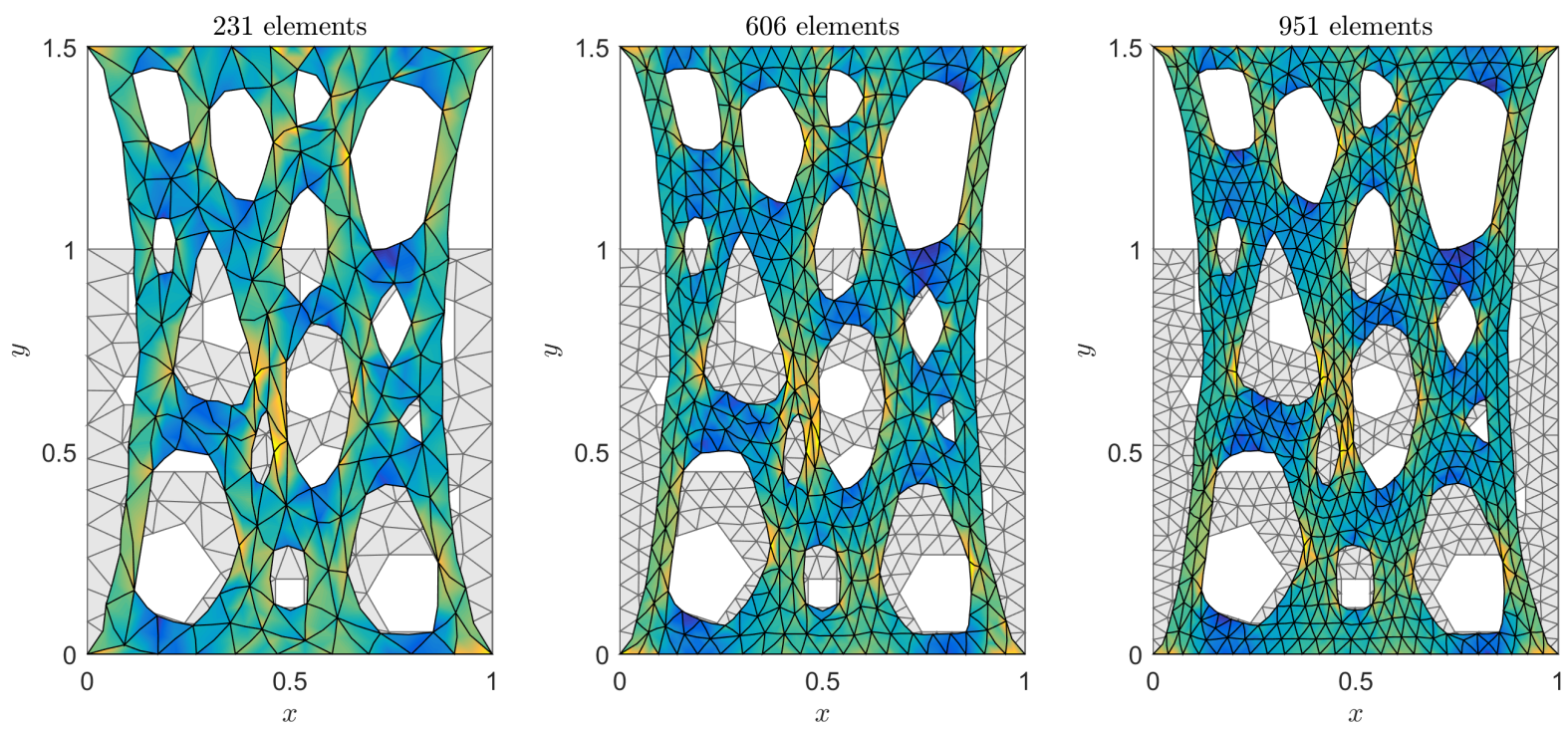

Figure 25: Deformed configurations of a plate with randomly distributed holes using $\mathrm{H} 2 \mathrm{c} 2 \mathrm{~d} \overline{2}$ and the meshes of Figure 23. The imposed vertical displacement at the upper boundary of the plate is $v=0.5 \mathrm{~mm}$ (50\% stretch). Colors indicate values of the norm of stress $\|\boldsymbol{P}\|$, where lighter colors correspond to higher values of $\|\boldsymbol{P}\|$.
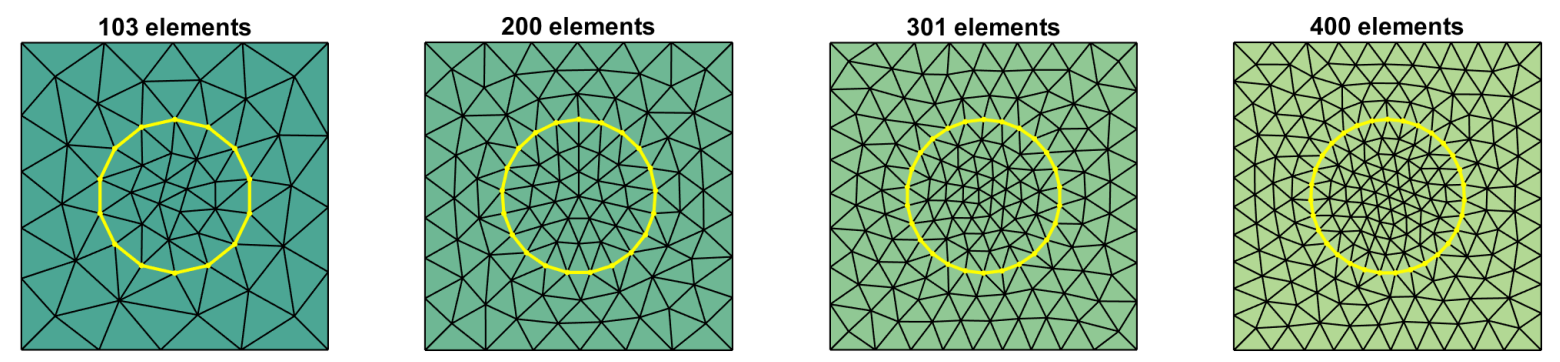

Figure 26: Four unstructured meshes for the heterogeneous plate example. The (yellow) circle indicates the interface between the plate and the circular inhomogeneity.

[18] T. Belytschko, J. S. J. Ong, W. K. Liu, and J. M. Kennedy. Hourglass control in linear and nonlinear problems. Comput. Methods in Appl. Mech. Eng., 43:251-276, 1984.

[19] S. Reese and P. Wriggers. A stabilization technique to avoid hourglassing in finite elasticity. Int. J. Numer. Meth. Engng., 48:79-109, 2000.

[20] J. C. Simo and F. Armero. Geometrically non-linear enhanced strain mixed methods and the method of incompatible modes. Int. J. Num. Methods Eng., 33:1413-1449, 1992.

[21] P. Wriggers. Mixed finite element methods - theory and discretization. In Mixed finite element technologies, pages 131-177. CISM Courses and Lectures, Springer-Verlag, Wien, 2009.

[22] N. C. Nguyen and J. Peraire. Hybridizable discontinuous galerkin methods for partial differential equations in continuum mechanics. J. Comput. Phys., 231:5955-5988, 2012. 

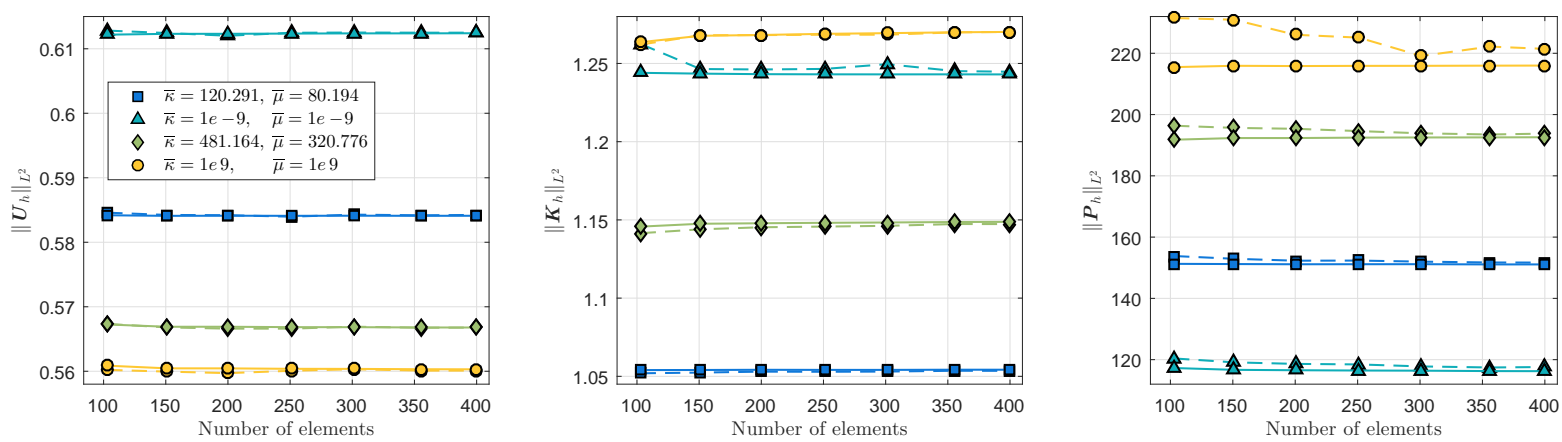

Figure 27: Tension of a heterogeneous plate: $L^{2}$-norms of displacement, displacement gradient, and stress for 100\% tension versus the number of elements in the mesh. The plate material parameters are $\kappa=120.291 \mathrm{~N} / \mathrm{mm}^{2}$ and $\mu=$ $80.194 \mathrm{~N} / \mathrm{mm}^{2}$ and those of the inhomogeneity $(\bar{\kappa}, \bar{\mu})$ are given in the legend. The dashed and the solid curves are obtained by using $\mathrm{H} 1 \mathrm{c} 1 \mathrm{~d} \overline{1}$ and $\mathrm{H} 2 \mathrm{c} 2 \mathrm{~d} \overline{2}$, respectively. The underlying meshes have 103, 151, 200, 251, 301, 356, and 400 elements.

[23] O. A. Ladyzhenskaya. The mathematical theory of viscous incompressible flow. Gordon and Breach Science Publishers, New York, 1969.

[24] I. Babuška. The finite element method with Lagrangian multipliers. Numer. Math., 20:179-192, 1973.

[25] F. Brezzi. On the existence, uniqueness and approximation of saddle-point problems arising from Lagrange multipliers. RAIRO Anal. Numér., pages 129-151, 1974.

[26] A. Ern and J. Guermond. Theory and Practice of Finite Elements. Springer-Verlag, New York, 2004.

[27] D. N. Arnold and R. Winther. Mixed finite elements for elasticity. Numer. Math., 92:401-419, 2002.

[28] E. Kröner. Allgemeine kontinuumstheorie der versetzungen und eigenspannungen. Arch. Rational Mech. Anal., 4:273-334, 1959.

[29] D. N. Arnold, R. S. Falk, and R. Winther. Finite element exterior calculus, homological techniques, and applications. Acta Numerica, 15:1-155, 2006.

[30] D. N. Arnold, R. S. Falk, and R. Winther. Finite element exterior calculus: from Hodge theory to numerical stability. Bul. Am. Math. Soc., 47:281-354, 2010.

[31] A. Angoshtari and A. Yavari. Differential complexes in continuum mechanics. Arch. Rational Mech. Anal., 216:193-220, 2015.

[32] A. Angoshtari and A. Yavari. Hilbert complexes of nonlinear elasticity. Submitted, 2016.

[33] P. A. Raviart and J. M. Thomas. A mixed finite element method for 2nd order elliptic problems. In Mathematical aspects of finite element methods (Proc. Conf., Consiglio Naz. delle Ricerche (C.N.R.), Rome, 1975), pages 292-315. Vol. 606 of Lecture Notes in Mathematics, Springer, Berlin, 1977.

[34] F. Brezzi, J. Douglas, Jr., and L. D. Marini. Two families of mixed finite elements for second order elliptic problems. Numer. Math., 47:217-235, 1985. 

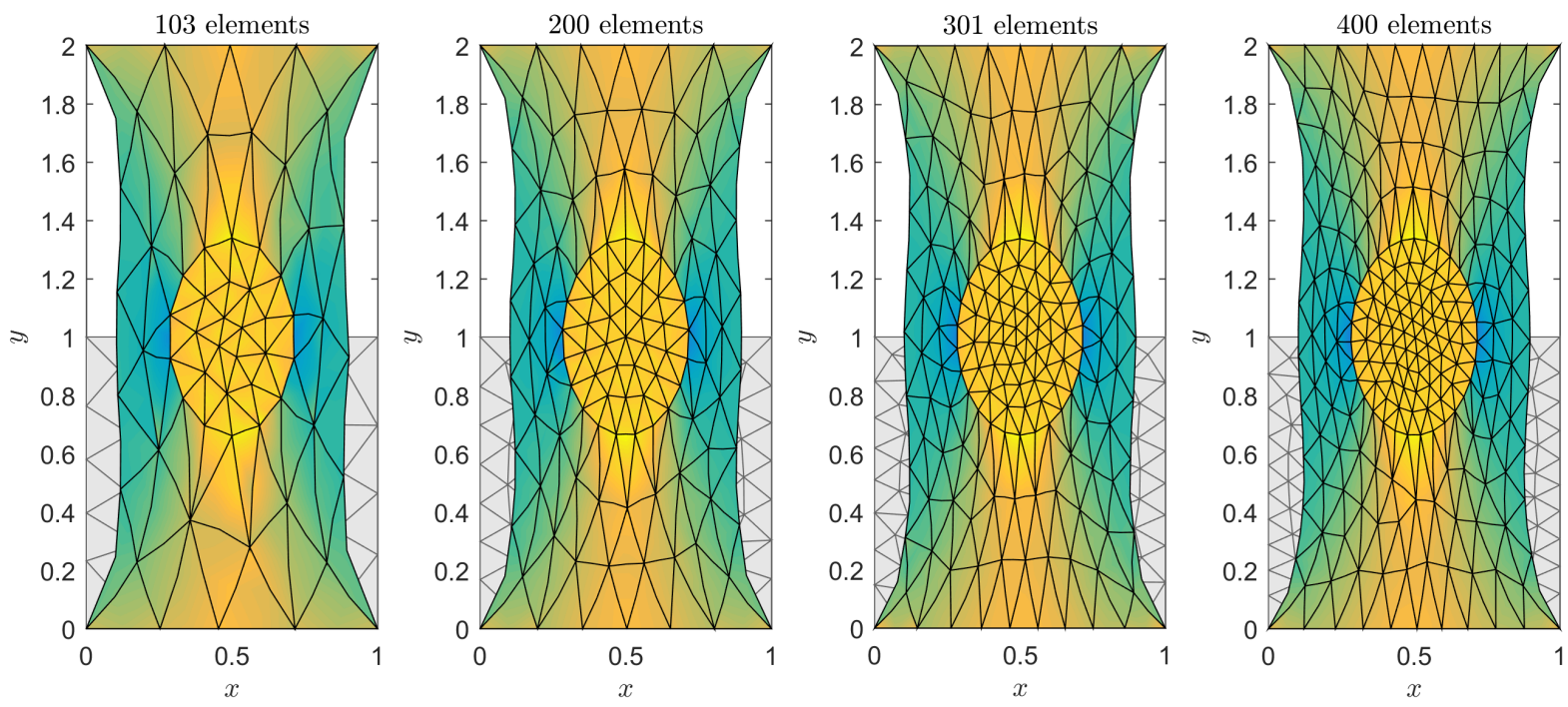

Figure 28: Deformed configurations of the heterogeneous plate under $100 \%$ stretch using $\mathrm{H} 2 \mathrm{c} 2 \mathrm{~d} \overline{2}$ and the meshes of Figure 26. The plate material parameters are $(\kappa, \mu)=(120.291,80.194) \mathrm{N} / \mathrm{mm}^{2}$ and those of the inhomogeneity are $(\bar{\kappa}, \bar{\mu})=(481.164,320.776) \mathrm{N} / \mathrm{mm}^{2}$. Colors indicate values of the norm of stress $\|\boldsymbol{P}\|$, where lighter colors correspond to higher values of $\|\boldsymbol{P}\|$.

[35] J. C. Nédélec. A new family of mixed finite elements in $\mathbb{R}^{3}$. Numer. Math., 50:57-81, 1986.

[36] G. Geymonat and F. Krasucki. Hodge decomposition for symmetric matrix fields and the elasticity complex in Lipschitz domains. Commun. Pure Appl. Anal., 8:295-309, 2009.

[37] T. Jakab, I. Mitrea, and M. Mitrea. On the regularity of differential forms satisfying mixed boundary conditions in a class of Lipschitz domains. Indiana Univ. Math. J., 58:2043-2071, 2009.

[38] J. L. Loday. Cyclic Homology. Springer-Verlog, Berlin, 1992.

[39] J. Ball and R. D. James. Fine phase mixtures as minimizers of energy. Arch. Ration. Mech. Anal., 100:13-52, 1987.

[40] A. Angoshtari and A. Yavari. The weak compatibility equations of nonlinear elasticity and the insufficiency of the Hadamard jump condition for non-simply connected bodies. Continuum Mech. Therm., 28:1347-1359, 2016.

[41] P. G. Ciarlet. The Finite Element Method for Elliptic Problems. North-Holland Publishing Co., Amsterdam, 1978.

[42] S. H. Christiansen and R. Winther. Smoothed projections in finite element exterior calculus. Math. Comp., 77:813-829, 2008.

[43] G. N. Gatica and N. Heuer. Conjugate gradient method for dual-dual mixed formulations. Math. Comp., 71:1455-1472, 2001.

[44] G. N. Gatica and N. Heuer. Minimum residual iteration for a dual-dual mixed formulation of exterior transmission problems. Numer. Linear Algebra Appl., 8:147-164, 2001. 



Figure 29: Deformed configurations of the heterogeneous plate under $100 \%$ stretch with different material properties for its inhomogeneity. Results are calculated using $\mathrm{H} 2 \mathrm{c} 2 \mathrm{~d} \overline{2}$ and the mesh has 200 elements. The plate material parameters are $(\kappa, \mu)=(120.291,80.194) \mathrm{N} / \mathrm{mm}^{2}$ and those of the inhomogeneity are written above the corresponding figure. Colors indicate values of the norm of stress $\|\boldsymbol{P}\|$, where lighter colors correspond to larger values of $\|\boldsymbol{P}\|$. Colors are normalized between figures such that the same colors in different figures indicate the same values.

[45] T. J. R. Hughes. The Finite Element Method: Linear Static and Dynamic Finite Element Analysis. Dover Publications, 2000.

[46] S. Reese. On the equivalence of mixed element formulations and the concept of reduced integration in large deformation problems. Int. J. Nonlin. Sci. Num., 3:1-33, 2002.

[47] U. Brink and E. Stein. A posteriori error estimation in large-strain elasticity using equilibrated local Neumann problems. Comput. Methods Appl. Mech. Engrg., 161:77-101, 1998. 